LA-10408-MS

UC-33A

Issued: September 198

LA--10408-MS

DE86 003282

\title{
General-Purpose Heat Source Safety Verification Test Series: SVT-7 Through SVT-10
}

\author{
T. G. George \\ D. Pavone
}

\section{DISCLAIMER}

This report was prepared as an account of work sponsored by an agency of the United States Government. Neither the United States Government nor any agency thereof, nor any of their employees, makes any warranty, express or implied, or assumes any legal liability or resporsibility for the accuracy, completeness, or usefulness of any information, apparatus, product, or process disclos:d, or represents that its use would not infringe privately owned rights. Reference herein to any specific commercial product, process, or service by trade name, trademark, manufacturer, or otherwise does not necessarily constitute or imply its endorsement, recommendation, or favoring by the United States Government or any agency thereof. The views and opinions of authors expressed herein do not necessarily state or reflect those of the United States Government or any agency thereof. 


\title{
GENERAL-PURPOSE HE IT SOURRE SAFETY VERIFICATION T EST SERIES: SVT-7 THROUGH SVT-10
}

by

\author{
T. G. George and D. Pavone
}

\begin{abstract}
The General-Purpose Heat Source (GPHS) is a modular component of the radioisotope thermoelectric generator that will supply power for the Galileo and Ulysses (formerly ISPM) space missions. The GPHS provides power by transmitting the her:t

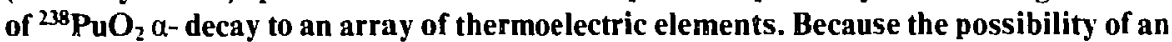
orbital abort always exists, the heat source was designed and construrted to minimize plutonia release in any accident environment. The Safety Verification Test (SVT) series was formulated to evaluate the effectiveness of GPHS plutonia containment after atmospheric reentry and Earth impact. The first report (covering SVT-1 through SVT-6) described the results of foc and side-on module impacts. This report describes module impacts at angles of $15^{\circ}$ and $30^{\circ}$.
\end{abstract}

\section{INTRODUCTION}

The General-Purpose Heat Source (GPHS) is a modular component of the radioisotope thermoelectric generator (RTG) that will provide power for the NASA Galileo and ESA Ulysses (formerly ISPM) space missions. The RTG generates electric power by using the heat of ${ }^{238} \mathrm{PuO}_{2}$ a-decay to create a temperature differential across a thermoelectric array. The Galileo mission will require two RTGs, and Ulysses will use a single RTG. Each RTG will contain 18 GPHS modules.

The GPHS module was designed by Los Alamos National Laboratory and modified by the General Electric Company, Inc., (system contractor for the RTG) to meet weight requirements of the Galileo and Ulysses missions. Each GPHS module contains four ${ }^{238} \mathrm{PuO}_{2}$ fuel pellets that provide a total thermai output of $250 \mathrm{~W}$. Each fuel pellet is encapsulated in a vented, DOP-26 iridium alloy shell. Two capsules are held in a Fineweave-Pierced Fabric (FWPF) graphite impact shell (GIS), and two GISs are contained within a FWPF graphite zeroshell. A schematic of the GPHS module is

Fineweave-Pierced Fabric 3-D carbon/carbon composite, a product of AVCO Systems Division, 20I Lowell St., Wilmington, MA 01887. shown in Fig. 1. Because the possibility of an orbital abort always exists, the GPHS module has been designed to minimize plutnniz zelease during atmospheric reentry and Earth impact.

Accident analyses have shown that in any orbital aboit, the GPHS modules would be released from the RTG early in reentry and would strike the Earth singly and intart.' The responses of GPHS modules with various processing histories to a variety of reentry and impact conditions were investigated in the Design Iteration Tests (DIT). With the conclusion of the DITs, emphasis shifted to venfying the survivability of GPHS modules in probable accident environments. The objective of the Safety Verification Test (SVT) series was to determine the response of the GPHS module and its components to atmospheric reentry, and Earth impact. ${ }^{2}$

The first six SVTs investigated the module response in a series of flat and side-on impacts; these tests were described in a previous report. ${ }^{3}$ Because the DIT results indicated a significant difference in the degree of capsule damage produced by various impact angles, the next four SVTs (tests 7 through 10) were designed to evaluate the system response to impact orientations of $\alpha=15^{\circ}$ and $30^{\circ}$ (schematics of the $15^{\circ}$ and $30^{\circ}$ impact orientations are shown in Figs. 2 and 3). 


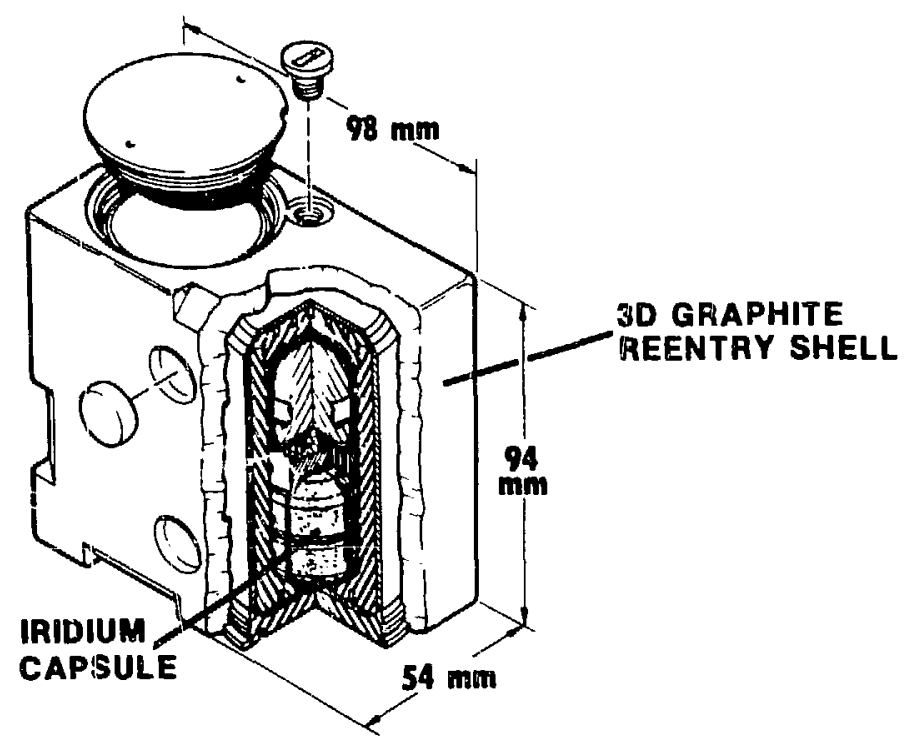

Fig. 1. The GPHS module.

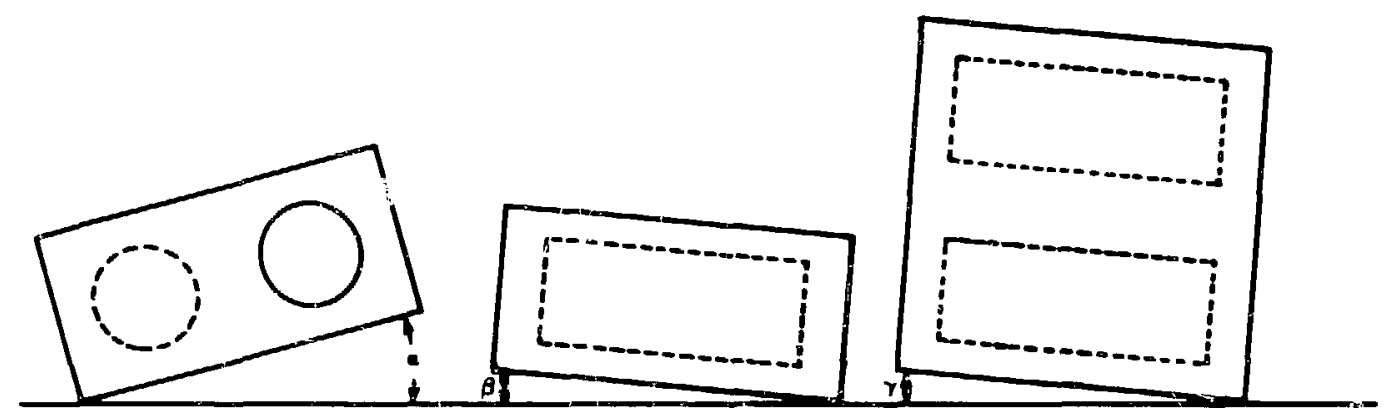

Fig. 2. The SVT -7 and SVT-8 test assemblies were impacted in the $a=15^{\circ}, \beta=0^{\circ}, \gamma=0^{\circ}$ module orientation.
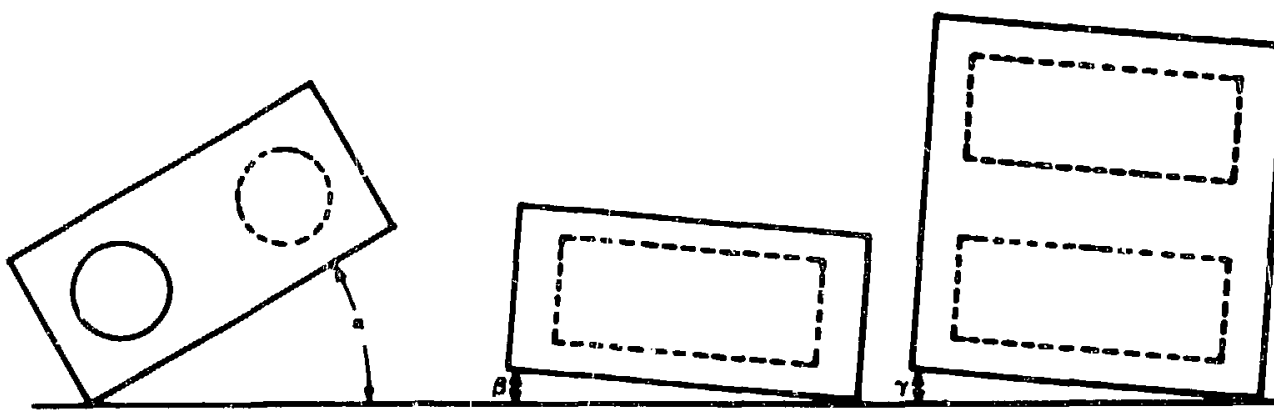

Fig. 3. The SVT-9 and SVI-10 test assemblies were impacted in the $\alpha=30^{\circ}, \beta=0^{\circ}, \gamma=0^{\circ}$ module orientation. 


\section{TEST PROGRAM}

The SVT test program was designed to simulate the atmospheric reentry and subsequent Earth impact experienced by a GPHS module in the aftermath of an orbital abort. The impact orientation of each test module was determined by a technical review board consisting of R. W. Zocher (Los Alamos National Laboratory) C. T. Bradshaw (GE), and R. W. Englehart (NUS). A total of ! 3 GPHS modules were allocated for the test series.

The test modules were assembled at the Monsanto Research Corporation (MRC) and were treated (to reduce the excess oxygen conient of the plutonia fuel) in the Mound Reduction and Monitoring Facility (MRMF). The treated modules were then subjected to two cycles of flight-acceptance vibration spectra and transient accelcrations, radiographed. and shipped to
Los Alamos. Components of the GPHS modules used in SVT-7 through SVT-10 are identified in Table I.

The plutonia fuel pellets required for the SVT impacts were fabricated and encapsulated in iridium at the Savannah River Plant (SRP). The iridium shclis were fabricated by the Mound Plant, from iridium alloy blanks supplied by the Oak Ridge National Laboratory (ORNL). Because of a process change, two classes of plutonia pellets (fired either in an $\mathrm{Ar}$ or $\mathrm{Ar} / \mathrm{O}_{2}$ atmosphere) were provided. Both types of pellets were considered to be flight quality; production histories of the fuel pellets used in SVT-7 through SVT-10 are presented in Table Il.

All graphite module components were also cumposed of fight-quality material. ORNL produced the carbonbonded carbon filament ( $C B C F$ ) graphite insulation and MRC provided the FWPF graphite aeroshells and GISs. To simulate the ablation resulting from an orbital decay

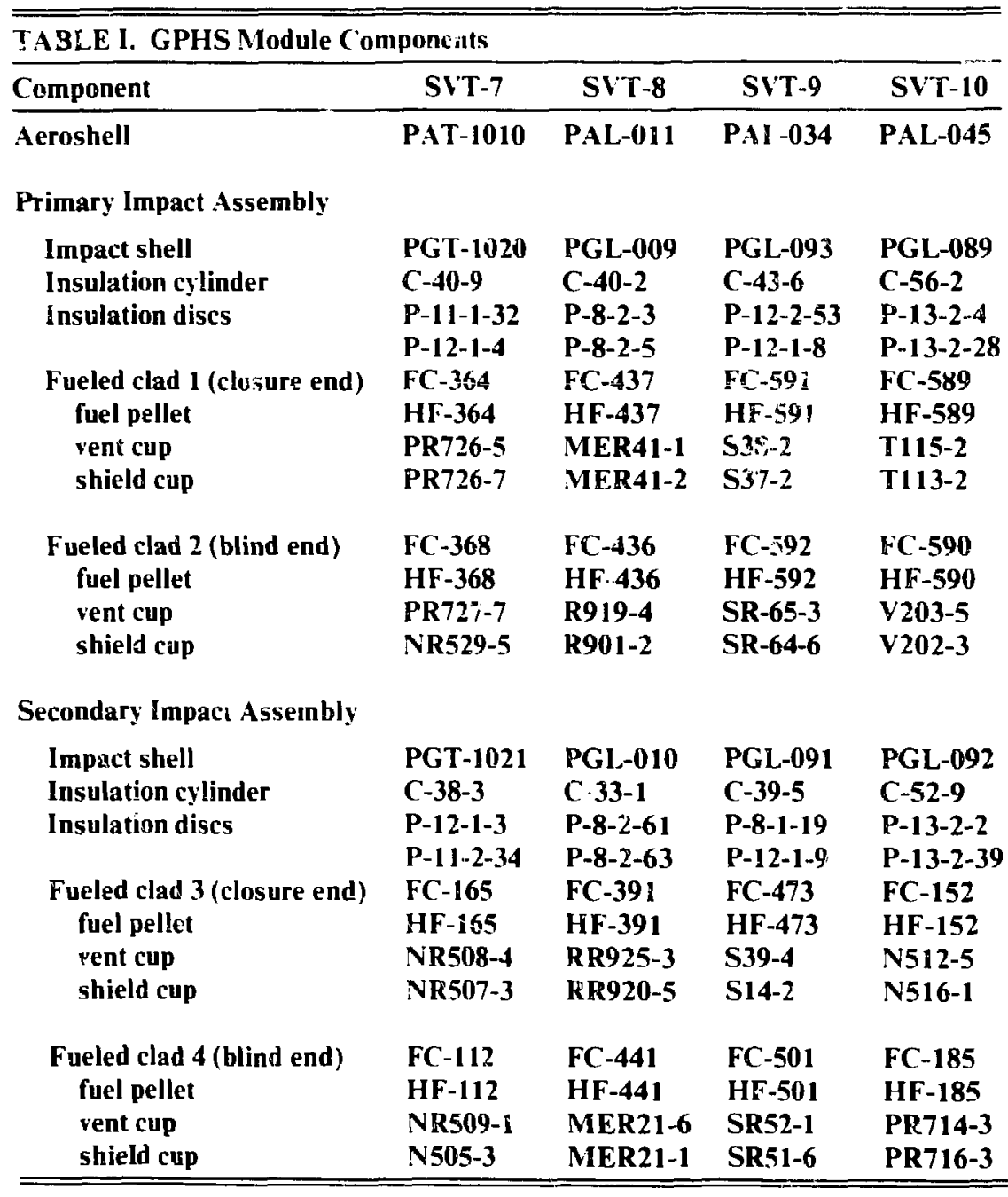




\begin{tabular}{|c|c|c|c|}
\hline \multirow[b]{2}{*}{ Pellet No. } & \multirow{2}{*}{$\begin{array}{c}\text { Weight } \\
\text { (g) }\end{array}$} & \multicolumn{2}{|c|}{ Processing Atmosphere } \\
\hline & & Ir & $\mathrm{Ar} / \mathrm{O}_{2}$ \\
\hline$H F-364$ & 149.7 & * & \\
\hline HF-368 & 150.3 & * & \\
\hline HF-165 & 148.0 & & * \\
\hline HF-112 & 148.0 & & * \\
\hline HF -437 & 149.8 & * & \\
\hline$H F-436$ & 150.1 & * & \\
\hline HF-391 & 150.3 & $*$ & \\
\hline $\mathrm{HF}-\mathbf{4 4 1}$ & 150.6 & * & \\
\hline HF-591 & 150.2 & * & \\
\hline HF -598 & 150.4 & * & \\
\hline $\mathrm{HF}-473$ & 150.4 & * & \\
\hline HF-501 & 150.3 & * & \\
\hline HF-589 & 150.1 & * & \\
\hline HF-590 & 150.4 & * & \\
\hline HF-152 & 148.9 & & * \\
\hline HF- 185 & 148.7 & & * \\
\hline
\end{tabular}

reentry: each FWPF aeroshell was machined (at Los Alamos) to remove $1.27 \mathrm{~mm}$ of graphite from all exth. nal surfaces (a thickness equivalent to twice the predicted ablation for an orbital decay reentry).

Each GPHS module contained two flight-quality and two non-flight-quality fucled clads. The flight-quality and non-flighti-quality clad designations were provided by SRP. Because cracking occurred in a number of carly test welds. SRP developed an ultrasonic nondestructive evaluation (NDE) technique that rated weld defects by comparison with a standard defect of known dimensions. Fucled clads with NDE ratings $\leq 8$ (arbitrary units) were designated as tlight quality. and capsules with NDE ratings $>8$ were reserved for terrestrial applications, where the abort environments are not as severe. In each test module. the flight-quality clads were loaded into a FW PF GIS (the primary impact assembly) and positioned in the module GIS cavity cxpected to receive the greatest deformation. Nonfligh:-quality clads were loaded into a GIS (secondary impact assembly) and positioned in the remaining GIS cavity.

\section{EXPERIME.VTAI, PROCEDLRES}

\section{A. Pretest Data}

The assembled test modules were shipped to Los Alamos in welded stainless stcel containers. When received. each container was radiographed to document the condition of the fuel pellets. After radiography the module was removed from the shipping container, disassembled, and macroscopically examined. A 1.5875 $\mathrm{mm}(0.0625$-in.) pyrometer sight hole was drilled into the cap of the primary impact assembly (PIA), and both GISs were loaded into a high-vacuum furnace for an aging heat treatment.

The aging treatment simulated the temperature of a GPHS module during the orbital decay of an aborted mission. The fueled clads used in SVT-7 through SVT- 10 were aged for 90 days at $1287^{\circ} \mathrm{C}$ : the predicted time interval for orbital decay of a Galileo abon is 90 days, and the operating temperature of an orbiting RTG is $1287^{\circ} \mathrm{C}$. Although an abort of the Ulysses mission would result in a 38-day orbital decay, a 90-day orbital decay was selected to represent both missions. ${ }^{4}$ Helium release from the SVT-8 and SVT-9 test components was menitored throughout the heat treatments; there was no evidence that any of the vents plugged during the 90-day period.

After aging, the GISs were prepared for reentry simulation. Holes for a split-junction thermocouple were drilled into the GIS caps. Each GIS was placed in an electron beam furnace and heated through an orbital decay reentry cycle (a schedule of the orbital decay reentry tempcratures is presented in Table III). After

\begin{tabular}{cccc}
\hline \hline \multicolumn{5}{c}{ TABLE III. Orbital } & Decay Reentry Schedule \\
\hline $\begin{array}{c}\text { Time } \\
(\mathrm{min})\end{array}$ & $\begin{array}{c}\text { Temperature } \\
\left({ }^{\circ} \mathrm{C}\right)\end{array}$ & $\begin{array}{c}\text { Time } \\
(\mathrm{min})\end{array}$ & $\begin{array}{c}\text { Temperature } \\
\left({ }^{\circ} \mathrm{C}\right)\end{array}$ \\
\hline 0.00 & 1294 & 10.25 & 1364 \\
0.50 & 1291 & 10.50 & 1371 \\
1.00 & 1285 & 10.75 & 1374 \\
1.50 & 1278 & 11.00 & 1372 \\
2.00 & 1269 & 11.25 & 1368 \\
2.50 & 1259 & 11.50 & 1360 \\
3.00 & 1248 & 11.75 & 1350 \\
3.50 & 1237 & 12.00 & 1335 \\
4.00 & 1228 & 12.25 & 1307 \\
4.50 & 1221 & 12.50 & 1275 \\
5.00 & 1216 & 12.75 & 1242 \\
5.50 & 1214 & 13.00 & 1214 \\
6.00 & 1212 & 13.25 & 1186 \\
6.50 & 1211 & 13.50 & 1158 \\
7.00 & 1213 & 13.75 & 1134 \\
7.50 & 1216 & 14.00 & 1114 \\
8.00 & $122 \%$ & 14.25 & 1090 \\
8.50 & 1246 & 14.50 & 1069 \\
8.75 & 1255 & 14.75 & 1047 \\
9.00 & 1271 & 15.00 & 1025 \\
9.25 & 1287 & 15.25 & 1005 \\
9.50 & 1312 & 15.50 & 983 \\
9.75 & 1333 & 15.75 & 964 \\
10.00 & 1349 & & \\
\hline \hline & & & \\
\hline \hline
\end{tabular}


reentry simulation, the GISs were radiegraphed a second time and prepared for impact lesting.

\section{B. Impact Testing}

After reentry simulation, the GISs were reloaded into the FWPF aeroshell. Holes were drilled through the aeroshell and impact shell walls to accommodate additional instrumentation. A calibrated split-junction thermocouple was placed in contact with one of the fight-quality clads. and two beaded-junction thermocouples were at tached to the surface of the PIA (the GIS containing the flight-quality clads). The module was then transferred tu the Los Alamos Isotope Fuels Impact Tes! Facility (a $185-\mathrm{mm}$ gas gun that contains the impacted test article within a sealed catch tube) ${ }^{5}$

All of the test modules were inpacted against 2 hardened steel plate. at a rominal velocity and temferature of $54 \mathrm{~m} / \mathrm{s}$ and $975^{\circ} \mathrm{C}$. The thermal output of the GPHS modulc was suflicient to Feimit self-heating to the test temzerature. Specific conditions for each tist are listed in $\mathrm{i}$ able IV.

\section{Postmortem Examination}

The principal objectives of the postmortem examination were to determine the quantity and particle size of the plutonia release. document the damage sustained by all test components. and characterize the iridium and plutonium impact responses.

After each test. the impacted module (contained within a sealed catch tube) was transferred to Wing 2 of the CMR building (at Los Alamos). and opened. Ali test components were photographed and the fueled clads were measured to determine the postimpact capsule strains. The size and location of all clad failures and cracks were recorded.

After macroscopic examination, the fueled clads were opened and the patterns of fuel fracture were photographeci. At least one unbreached capsule was selected for a particle size analysis; this capsule was opened under water to prevent the loss of small fuel particles.
The capsules were defueled and specimens of each fuel pellet were taken for metallographic and chemical analyses. Fuel pellets selected for particle size analyses were not sampled until after the sieve analyses.

The iridium clads were also sampled to provide specimens for metallographic and chemicil analyses. The SVT test plan required that each primary clad the capsules contained within the P(A) be sampled to provide axial and transverse sections of the vent and weld shield cups, a vent cross section, and a weld (single pass region) cross section. In addition, all clad failures and areas of sevire localized deformation were sectioned for metallugraphy. The secondary clads wert also sampled when there was clad failure or severe defornation.

All graphite test components, including the calch tube debris, were analyzed to determine the amumin and particle size of plutonia release. The graphite iterushell. impact shells, and all large pieces of CBCF insulation were ultrasonically cleaned in ethyl alcohol. dried, and burned in air at $85010900^{\circ} \mathrm{C}$. The resulting ash was dissolved in acid and radicanalyzed for plutonium. The plutonium content of the graphite test components was assumed to be present as small plutonia fragments $(<10$ $\mu \mathrm{m})$ trapped within the pores of the FWPF graphite.

The ultrasonic cleaning solutior. was combined with the debris remaining in the catch tube, dried, and burned in oxygen in a plasma-assisted combustion furnace. The plasma-assisted furnace was used because its low operating temperature $\left(\cong 50^{\circ} \mathrm{C}\right)$ minimized particle agg!omeration (sintering). The resulting ash was macroscopically examined, and all large pieces of noncombustible material (such as thermocoupie wiring, alumina insulation, etc.) were removed. The remaining material was then sized and submitted for radioanalysis.

The plu'tonia release in SVT-8 was determined by a different method. The large graphite components were processed as usual, but the ultrasonic cleaning solution and catch tube debris were combined, dried, sized into three fractions $(-10 \mu \mathrm{m}, 10 \mu \mathrm{m}$ to $-420 \mu \mathrm{m}$, and 420 $\mu \mathrm{m}$ and above), burned at high temperature ( 850 to $900^{\circ} \mathrm{C}$ ), and radioanalyzed. It was felt that the time required for low-temperature ashing was not warranted for a plutonia release resulting from the failure of a nonflight-quality weld.

\begin{tabular}{llccc}
\hline \hline TABLE IV. Test Parameters & & & \\
\hline Test & Module Orientation & $\begin{array}{c}\text { Temperature } \\
\left({ }^{\circ} \mathrm{C}\right)\end{array}$ & $\begin{array}{c}\text { Velocity } \\
(\mathrm{m} / \mathrm{s})\end{array}$ & $\begin{array}{c}\text { Target } \\
\text { Material }\end{array}$ \\
\hline SVT-7 & $\alpha=15^{\circ}, \beta=0^{\circ}, \gamma=0^{\circ}$ & 975 & 53.5 & steel \\
SVT-8 & $\alpha=15^{\circ}, \beta=0^{\circ}, \gamma=0^{\circ}$ & 975 & 54.6 & steel \\
SVT-9 & $\alpha=30^{\circ}, \beta=0^{\circ}, \gamma=0^{\circ}$ & 975 & 54.6 & steel \\
SVT-10 & $\alpha=30^{\circ}, \beta=0^{\circ}, \gamma=0^{\circ}$ & $\mathbf{9 7 5}$ & 54.3 & steel \\
\hline \hline
\end{tabular}




\section{RESLLTS}

The individual tests and postmortem examinations are summarized below. Analytical results are tabulated as follows:

Table V. SVT Summaries

Table VI. SVT Fuel Release Data

Table V1l. Sieve Analyses of Fuel in Unbreached SVT Clads
Table VIll. Iridium Grain Size of Selected SVT Capsules

Table IX. SVT Iridium AES Analyses

Table X. Spectrographic Analyses of SVT Iridium Clads

Table X1. SVT Plutonia Analýses

\begin{tabular}{|c|c|c|c|c|c|c|c|c|c|}
\hline \multirow[b]{3}{*}{ Test } & \multirow{3}{*}{$\begin{array}{c}\text { Reentry } \\
\text { Mode }\end{array}$} & \multirow{3}{*}{$\begin{array}{c}\text { Impact } \\
\text { Orientstion }\end{array}$} & \multirow{3}{*}{$\begin{array}{l}\text { Fueled } \\
\text { Clads }\end{array}$} & \multirow{3}{*}{$\begin{array}{c}\text { Clad } \\
\text { NDE } \\
\text { Values }\end{array}$} & \multirow{2}{*}{\multicolumn{3}{|c|}{ Postimpact Capsule Strains }} & \multicolumn{2}{|c|}{ Failures } \\
\hline & & & & & & & & & Total Area \\
\hline & & & & & Diam (\%) & Height (\%) & Axial (\%) & Nunber & $\left(\mathrm{mm}^{2}\right)$ \\
\hline \multirow[t]{4}{*}{ SYT-7 } & Orbital & $a=15^{\circ}$ & FC -364 & 2.0 & +9.9 & -13.5 & +5.3 & 1 & \\
\hline & Decay & $\beta=0^{\circ}$ & FC -368 & 1.6 & +9.5 & -9.9 & +5.4 & 0 & 53.0 \\
\hline & & $\gamma=0^{\circ}$ & FC -165 & 13.0 & +10.7 & -10.0 & +5.7 & $?$ & \\
\hline & & & FC-112 & 11.0 & +8.0 & -9.5 & +4.0 & 2 & \\
\hline \multirow[t]{4}{*}{ SVT-8 } & Orbital & $\alpha=15^{\circ}$ & FC $-4 \mid 36$ & 1.0 & +6.2 & -5.2 & +3.6 & 0 & \\
\hline & Decay & $\beta=0^{\circ}$ & FC -437 & 1.0 & +8.8 & -7.9 & +5.4 & 0 & \\
\hline & & $\gamma=0^{\circ}$ & FC-391 & 9.8 & +10.4 & -10.0 & +6.5 & 1 & $54.0^{n}$ \\
\hline & & & $F C-441$ & 9.6 & +8.9 & -8.9 & +5.3 & 0 & \\
\hline \multirow[t]{4}{*}{ SVT-9 } & Orbital & $\mathbf{a}=30^{\circ}$ & FC -592 & 3.0 & +6.0 & -6.1 & +2.4 & 0 & \\
\hline & Decay & $\beta=0^{\circ}$ & FC-591 & 1.6 & +7.1 & -8.9 & +3.7 & 0 & 0 \\
\hline & & $\gamma=0^{\circ}$ & FC -473 & 10.3 & +6.0 & -5.1 & +2.0 & $\mathbf{0}$ & \\
\hline & & & FC-501 & 11.4 & +6.7 & -5.7 & +4.3 & 0 & \\
\hline \multirow[t]{4}{*}{ SIT-10 } & Orbital & $\alpha=30^{\circ}$ & FC-589 & 1.7 & +4.2 & -3.5 & +2.5 & 0 & \\
\hline & Decay & $\beta=0^{\circ}$ & FC -590 & 3.7 & +5.4 & -6.6 & +5.0 & $\mathbf{0}$ & \\
\hline & & $\gamma=0^{\circ}$ & FC-152 & 11.0 & +6.9 & -7.3 & +3.6 & $\mathbf{0}$ & 0 \\
\hline & & & $\mathrm{F} C-185$ & 1.5 & +6.5 & -6.8 & +3.7 & 0 & \\
\hline
\end{tabular}

"Capsule FC-391 failed along the weld centerline; the weld shield band remained in place and limited fuel release.

\begin{tabular}{|c|c|c|c|c|}
\hline \multicolumn{5}{|c|}{ TABLE V1. SVT Fuel Releasa Data" } \\
\hline \multirow[b]{2}{*}{ Test } & \multirow{2}{*}{$\begin{array}{c}\text { Impact } \\
\text { Orientation }\end{array}$} & \multicolumn{3}{|c|}{${ }^{238}$ Pu Release (g) } \\
\hline & & Total & $<10-\mu m$ fraction & $>10-\mu m$ fraction \\
\hline SVT-7 & $\begin{array}{l}\boldsymbol{a}=15^{\circ} \\
\boldsymbol{\beta}=0^{\circ} \\
\gamma=0^{\circ}\end{array}$ & 0.2227 & 0.1390 & 0.0837 \\
\hline SVT-8 & $\begin{array}{l}\boldsymbol{\alpha}=15^{\circ} \\
\boldsymbol{\beta}=0^{\circ} \\
\gamma=0^{\circ}\end{array}$ & 0.0860 & 0.0687 & 0.0173 \\
\hline SVT-9 & $\begin{array}{l}\mathbf{a}=3^{\circ} \\
\beta=0^{\circ} \\
\gamma=0^{\circ}\end{array}$ & 0.0024 & 0.0024 & 0 \\
\hline SIT-10 & $\begin{array}{l}\dot{\alpha}=30^{\circ} \\
\beta=0^{\circ} \\
\gamma=0^{\circ}\end{array}$ & 0.0026 & 0.0026 & $\mathbf{0}$ \\
\hline
\end{tabular}

"No clad failures occurred in SVT-9 or SVT-10; all fuel release resulted from transport through the capsule vents. 


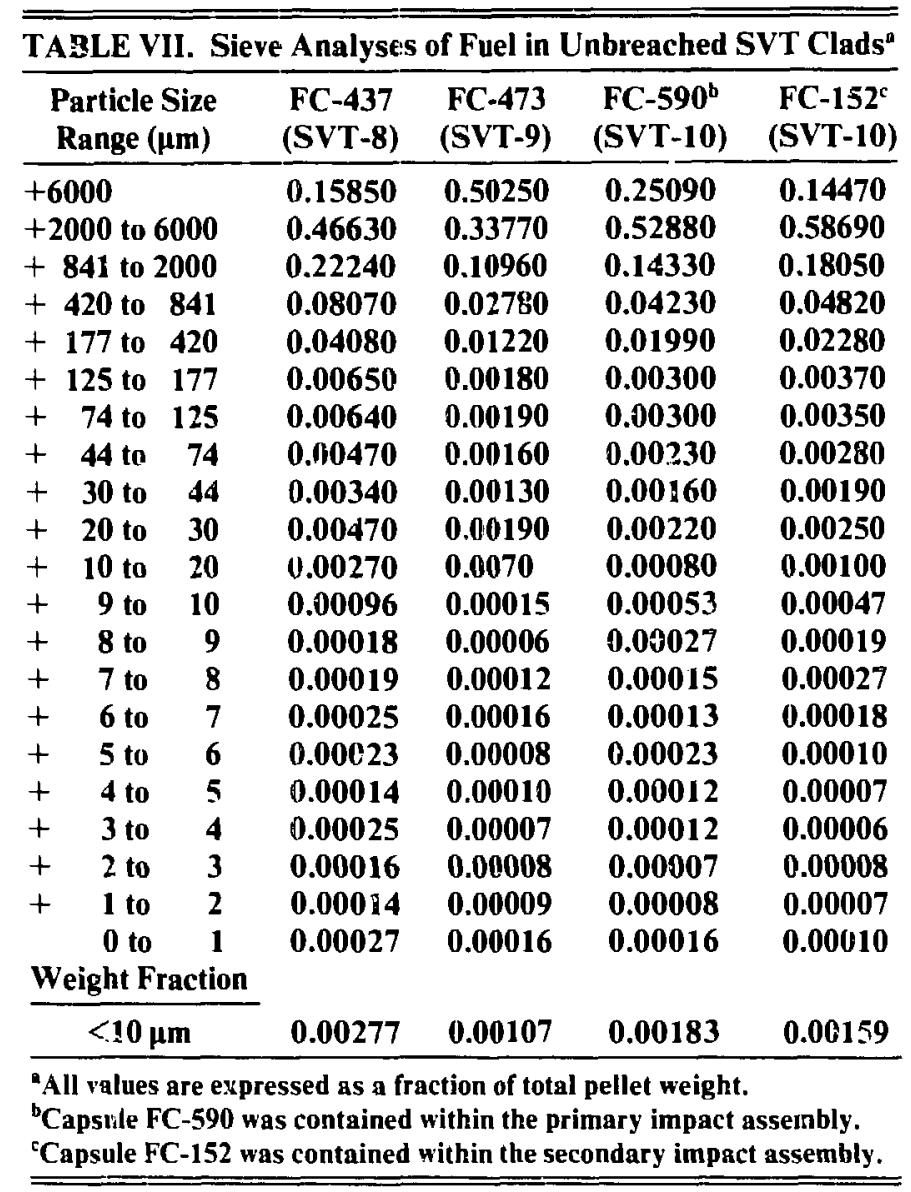

\section{A. SVT-7}

The test module was impacted $c n$ a steel target at 53.5 $\mathrm{m} / \mathrm{s}$ and $975^{\circ} \mathrm{C}$. The impact orientation was $\alpha=15^{\circ}$. $\beta=0^{\circ}, \gamma=0^{\circ}$. Postimpact examination revealed that the aeroshell fractured along the axial contact lines of the leading GIS (Fig. 4). The lead narrow face of the aeroshell separated from the aeroshell body and broke into two pieces. Impact shell damage was moderate, similar to that observed in $\alpha=0^{\circ}, \beta=0^{\circ}, \gamma=0^{\circ}$ impacts. Axial cracks (approximately $80 \%$ of the GIS length) occurred on the impact faces of both GISs.

Clad damage was extensive. Breaching cracks, apparently caused by the differential displacement of large fuel fragments, were observed on the impact face of capsule FC-364 (Fig. 5) and on the trailing face of FC-165 (Fig. 6). A centerline weld crack (10 mm long) was also observed on capsule FC- $165,90^{\circ}$ to the impact face.

Small fractures were observed on the exteriors of capsules FC-368 and FC-112, and two small weld cracks perpendicular to the weld centerline were found on the trailing face of capsule FC-112 (Fig. 7). A small fracture was also observed on the FC-368 vent cup radius (Fig. 8). However, full penetration of the crack on the FC -368 vent cup was not confirmed by subsequent metallography.

Examination of wall sections removed from the FC-364 vent and weld shield cups (Figs. 9 and 10) revealed a brittle intergranular failure. The breachı.ng crack apparently resulted from the differential displacement of a large fuel fragment. As is frequently observed in this type of breach, the fracture occurred in a reverse bend region adjacent to the fuel fragment imprint. The limited ductility of DOP-26 iridium and its dependence on strain state are illustrated by the reduction in wall thickness at the fracture ( $5 \%$ in the vent cup. $9 \%$ in the shield cup) and at the point of fuel fragment indentation (approximately $18 \%$ in both cups).

Metallographic examination of the breach in capsule FC. 165 revealed the same intergranular crack morphology observed in the FC-364 failure (Figs. 1] and 12). Because the FC-I65 breach occurred on the trailing capsule face, the fuel fragment imprint was not as sharply defined as it was in the FC-364 failure. However, as in capsule FC-364, the FC-165 breach 


\begin{tabular}{|c|c|c|c|c|c|}
\hline \multicolumn{6}{|c|}{ TABLE VIll. Iridium Grain Size of Selected SVT Capsules" } \\
\hline \multirow{2}{*}{$\begin{array}{l}\text { Fueled } \\
\text { Clad }\end{array}$} & \multirow[b]{2}{*}{ Test } & \multirow{2}{*}{$\begin{array}{c}\text { Iridium } \\
\text { Cup }\end{array}$} & \multicolumn{2}{|c|}{ Grain Size } & \multirow[b]{2}{*}{ Comments } \\
\hline & & & Transverse & Axial & \\
\hline \multirow[t]{2}{*}{ FC-165 } & SIT-7 & PR507-3 & 21 & 16 & $\begin{array}{l}\text { Large interual grains } 20 \text { to } \\
30 \% \text { of thickness }\end{array}$ \\
\hline & & PR508-4 & 16 & 13 & $\begin{array}{l}\text { Large internal grains } 20 \text { to } \\
30 \% \text { of thickness }\end{array}$ \\
\hline \multirow[t]{2}{*}{ FC-364 } & SIT-7 & PR726-5 & 15 & 15 & $\begin{array}{l}\text { Large internal grains } 25 \text { to } \\
30 \% \text { of thickness }\end{array}$ \\
\hline & & $P R=26-7$ & 16 & 15 & $\begin{array}{l}\text { Large internal grains } 25 \text { to } \\
30 \% \text { of thickness }\end{array}$ \\
\hline \multirow[t]{2}{*}{ FC- -368} & SIT-7 & PR727-7 & 15 & 17 & $\begin{array}{l}\text { Large internal grains } 20 \text { to } \\
35 \% \text { of thickness }\end{array}$ \\
\hline & & MR529-5 & 14 & 13 & $\begin{array}{l}\text { Large inteinal grains } 25 \text { to } \\
30 \% \text { of thickness }\end{array}$ \\
\hline \multirow[t]{2}{*}{ FC.391 } & SVT-8 & RR920-5 & 15 & $N^{b}$ & Typical microstructure \\
\hline & & RR925-3 & ND & 13 & Typical microstructure \\
\hline \multirow[t]{2}{*}{$F C-4.36$} & SVT-8 & R901-2 & 13 & 13 & $\begin{array}{l}\text { Large internal grains } 20 \text { to } \\
\mathbf{3 0 \%} \text { of thickness }\end{array}$ \\
\hline & & R919-4 & 14 & 12 & $\begin{array}{l}\text { Large internal grains } 20 \text { to } \\
30 \% \text { of thickness }\end{array}$ \\
\hline \multirow[t]{2}{*}{ FC-437 } & SVT-8 & MER41-1 & 14 & 13 & $\begin{array}{l}\text { Large internal grains } 20 \text { to } \\
30 \% \text { of thickness }\end{array}$ \\
\hline & & MER41-2 & 13 & 13 & $\begin{array}{l}\text { Large internal grains } 20 \text { to } \\
\mathbf{3 0} \% \text { of thickness }\end{array}$ \\
\hline \multirow[t]{2}{*}{ FC -473} & SVT-9 & $539-4$ & 13 & 15 & $\begin{array}{l}\text { Large internal grains up te } \\
25 \% \text { of thickness, and } \\
\text { isolated large external } \\
\text { grains }\end{array}$ \\
\hline & & S14-2 & 16 & 17 & $\begin{array}{l}\text { Large internal grains up to } \\
25 \% \text { of thickness }\end{array}$ \\
\hline \multirow[t]{2}{*}{$5 C-501$} & SVT-9 & SR51-6 & 15 & 18 & $\begin{array}{l}\text { Large internal grains } 20 \text { to } \\
30 \% \text { of thickness }\end{array}$ \\
\hline & & SR52-1 & 14 & 14 & $\begin{array}{l}\text { Large internal grains up to } \\
25 \% \text { of thickness, and } \\
\text { isolated large external } \\
\text { grains }\end{array}$ \\
\hline \multirow[t]{2}{*}{ FC-152 } & SVT-10 & N512-5 & 16 & ND & Typical microstructure \\
\hline & & N516-1 & 14 & ND & Typical microstructure \\
\hline \multirow[t]{2}{*}{ FC. 589} & SVT-10 & T113-: & 14 & 11 & $\begin{array}{l}\text { Numerous delaminations } \\
\text { observed along inner } \\
\text { surface }\end{array}$ \\
\hline & & T115-2 & 14 & 12 & $\begin{array}{l}\text { Numerous delaminations } \\
\text { observed along inner } \\
\text { surface }\end{array}$ \\
\hline \multirow[t]{2}{*}{ FC-590 } & SVT-10 & $v 202-3$ & 4 & 10 & $\begin{array}{l}\text { Isolated large grains } \\
\text { observed throughout the } \\
\text { microstructure }\end{array}$ \\
\hline & & V203-5 & 11 & 11 & Typical microstructure \\
\hline
\end{tabular}

aqrain sizes are given as an average of grains/0.j35-mm nominal wall thickness.

bprot determined. 


\begin{tabular}{|c|c|c|c|c|c|c|c|c|c|}
\hline \multirow[b]{2}{*}{ Specimen } & \multicolumn{4}{|c|}{$\mathbb{T h}_{65} / \mathbf{I r}_{229}$} & \multirow{2}{*}{$\begin{array}{c}\mathbf{P}_{120} / \mathbf{I r}_{229} \\
(\mathrm{Av})\end{array}$} & \multirow{2}{*}{$\begin{array}{c}C_{270} / I_{r_{229}} \\
(\mathrm{Av})\end{array}$} & \multirow{2}{*}{$\begin{array}{c}\mathrm{O}_{510} / \mathrm{Ir}_{229} \\
(\mathrm{Av}) \\
\end{array}$} & \multirow{2}{*}{$\begin{array}{c}S_{150} / I_{r_{229}} \\
(A v)\end{array}$} & \multirow{2}{*}{$\begin{array}{c}\mathrm{Cl}_{180} / \mathrm{Ir}_{229} \\
(\mathbf{A v})\end{array}$} \\
\hline & Int. & Center & Ext. & (Av) & & & & & \\
\hline FC-364 $\mathrm{V}^{\mathrm{a}}$-cup 1 & 0.27 & 0.59 & 0.54 & 0.47 & 0.02 & 0.52 & 0.80 & 0.02 & 0.10 \\
\hline$V$-cup 2 & 0.27 & 6.53 & 0.42 & 0.41 & 0.03 & 0.33 & 0.63 & $\mathbf{N D}^{\mathrm{h}}$ & 0.09 \\
\hline$S^{c}-$ cup 1 & 0.21 & 0.68 & 0.62 & 0.50 & ND & 0.14 & 0.39 & ND & ND \\
\hline 5-cup 2 & 0.34 & 0.68 & 0.48 & 0.50 & ND & 0.19 & 0.35 & 0.01 & ND \\
\hline FC-358 V-cup 1 & 0.31 & 0.54 & 0.63 & 0.50 & ND & 0.78 & 0.98 & $\mathbf{V S}^{\mathbf{d}}$ & 0.02 \\
\hline V-cup 2 & 0.70 & 0.84 & 0.59 & 0.71 & ND & 0.10 & 0.22 & ND & ND \\
\hline S-cup 1 & 0.29 & 0.85 & 0.68 & 0.61 & 0.07 & 0.16 & 0.34 & 0.01 & ND \\
\hline S-cup 2 & 0.19 & 0.55 & 0.64 & 0.46 & 0.04 & 0.20 & 0.30 & 0.02 & ND \\
\hline FC-165V-cup 1 & 0.37 & 0.53 & 0.13 & 0.34 & ND & 0.51 & 0.95 & VS & ND \\
\hline$V-\operatorname{cop} 2$ & 0.06 & 0.44 & 0.37 & 0.29 & ND & 5.68 & 1.73 & VS & ND \\
\hline S-cup 1 & 0.19 & 0.35 & 0.40 & 0.21 & ND & 1.08 & 1.14 & vS & 0.12 \\
\hline S-cup 2 & 0.68 & 1.47 & 0.45 & 0.87 & ND & 0.08 & 0.10 & ND & ND \\
\hline FC-436 V-cup 1 & 0.14 & 0.67 & 0.39 & 0.40 & ND & 0.02 & 0.51 & 0.02 & $N D$ \\
\hline$V$-cup 2 & 0.18 & 0.39 & 0.46 & 0.34 & ND & 0.08 & 0.92 & 0.05 & 0.16 \\
\hline S-cup 1 & 0.31 & 0.43 & 0.44 & 0.39 & ND & 0.05 & 1.32 & 0.05 & 0.14 \\
\hline S-cup 2 & 0.39 & 0.92 & 0.60 & 0.64 & ND & 0.03 & 0.26 & 0.01 & 0.01 \\
\hline FC-437 V-cup 1 & 0.39 & 0.82 & 0.77 & 0.66 & ND & ND & ND & ND & ND \\
\hline V-cup 2 & 0.15 & 0.50 & 0.59 & 0.41 & ND & 0.05 & 0.54 & ND & ND \\
\hline S-cup 1 & 0.21 & 0.96 & 0.62 & 0.60 & ND & ND & 0.13 & ND & ND \\
\hline S-cup 2 & 0.11 & 0.40 & 0.33 & 0.28 & ND & 0.06 & 0.68 & ND & ND \\
\hline FC-473 V-cup 1 & 0.08 & 0.48 & 0.54 & 0.37 & 0.03 & 0.57 & 0.99 & 0.06 & 0.09 \\
\hline Y-cup 2 & 0.38 & 0.45 & 0.42 & 0.42 & ND & 0.81 & 1.13 & 0.12 & ND \\
\hline S-cup 1 & 0.05 & 0.51 & 0.37 & 0.31 & 0.07 & 0.40 & 0.78 & ND & 0.03 \\
\hline S-cup 2 & 0.23 & 0.63 & 0.51 & 0.46 & 0.08 & 0.46 & 0.91 & 0.04 & ND \\
\hline FC-501 V-cup 1 & 0.24 & 0.38 & 0.59 & 0.40 & 0.02 & 1.20 & 0.88 & 0.18 & 0.28 \\
\hline V-cup 2 & 0.09 & 0.24 & 0.15 & 0.16 & ND & 0.77 & 1.32 & vS & 0.03 \\
\hline S-cup 1 & 0.41 & 0.37 & 0.62 & 0.47 & 0.05 & 0.88 & 0.93 & 0.04 & 0.04 \\
\hline S-cup-2 & 0.31 & 0.50 & 0.56 & 0.46 & ND & 0.74 & 1.04 & vs & ND \\
\hline FC-589 V-cup 1 & 0.05 & 0.44 & 0.38 & 0.29 & ND & 0.05 & 0.57 & ND & ND \\
\hline$V$ cup-2 & 0.04 & 0.62 & 0.51 & 0.39 & ND & 0.07 & 0.65 & 0.06 & 0.02 \\
\hline$S$ cup-1 & 0.04 & 0.37 & 0.37 & 0.26 & ND & 0.05 & 0.67 & 0.03 & 0.06 \\
\hline S cup-2 & ND & 0.30 & 0.28 & 0.19 & ND & 0.04 & 0.18 & ND & ND \\
\hline FC-590 V-cup 1 & 0.04 & 0.16 & 0.48 & 0.23 & ND & 0.08 & 1.24 & 0.87 & 0.14 \\
\hline$V$ cup-2 & 0.04 & 0.55 & 0.54 & 0.38 & ND & 0.07 & 0.96 & 0.82 & 0.05 \\
\hline S cup-1 & 0.03 & 0.40 & 0.20 & 0.21 & ND & 0.05 & 0.94 & 0.76 & 0.15 \\
\hline$S$ cup-2 & 0.04 & 0.08 & 0.47 & 0.20 & ND & 0.08 & 0.76 & 0.10 & 0.21 \\
\hline \multicolumn{10}{|c|}{$\begin{array}{l}\text { avent cup. } \\
\text { b None detected. } \\
\text { 'Shield cup. } \\
\text { dd Very strong elemental peak. }\end{array}$} \\
\hline
\end{tabular}




\begin{tabular}{|c|c|c|c|c|c|c|c|c|c|c|}
\hline \multirow[b]{2}{*}{ Capsule } & \multirow[b]{2}{*}{ Cup } & \multicolumn{9}{|c|}{ Selected Elements $(\mathrm{ppm})$} \\
\hline & & $\mathrm{Cu}$ & $\mathrm{Mg}$ & $\mathrm{Ca}$ & Al & $\mathbf{S i}$ & $\mathrm{Cr}$ & $\mathrm{Fe}$ & $\mathrm{Ni}$ & $\mathbf{P t}$ \\
\hline FC-364 & $\begin{array}{l}\text { PR726-5 } \\
\text { PR726-7 }\end{array}$ & $\begin{array}{r}15 \\
5\end{array}$ & $\begin{array}{l}N^{b} \\
\text { ND }\end{array}$ & $\begin{array}{l}\text { ND } \\
\text { ND }\end{array}$ & $\begin{array}{l}50 \\
50\end{array}$ & $\begin{array}{l}\text { ND } \\
\text { ND }\end{array}$ & $\begin{array}{l}30 \\
20\end{array}$ & $\begin{array}{l}200 \\
200\end{array}$ & $\begin{array}{l}30 \\
30\end{array}$ & $\begin{array}{l}\text { ND } \\
\text { ND }\end{array}$ \\
\hline FC-368 & $\begin{array}{r}\text { PR7:7-7 } \\
\text { NR529-5 }\end{array}$ & $\begin{array}{l}5 \\
5\end{array}$ & $\begin{array}{r}\text { ND } \\
3\end{array}$ & $\begin{array}{l}\text { ND } \\
\text { ND }\end{array}$ & $\begin{array}{l}50 \\
50\end{array}$ & $\begin{array}{l}20 \\
10\end{array}$ & $\begin{array}{l}40 \\
30\end{array}$ & $\begin{array}{l}200 \\
200\end{array}$ & $\begin{array}{l}50 \\
50\end{array}$ & $\begin{array}{l}\mathbf{3 0} \\
\mathbf{5 0}\end{array}$ \\
\hline FC-165 & $\begin{array}{l}\text { NR508.4 } \\
\text { NR507..3 }\end{array}$ & $\begin{array}{l}15 \\
15\end{array}$ & $\begin{array}{l}\text { ND } \\
\text { N? }\end{array}$ & $\begin{array}{l}\text { ND } \\
\text { ND }\end{array}$ & $\begin{array}{l}50 \\
70\end{array}$ & $\begin{array}{r}10 \\
\text { ND }\end{array}$ & $\begin{array}{l}30 \\
30\end{array}$ & $\begin{array}{l}200 \\
200\end{array}$ & $\begin{array}{l}30 \\
30\end{array}$ & $\begin{array}{r}\text { ND } \\
30\end{array}$ \\
\hline FC-391 & $\begin{array}{l}\text { RR920-5 } \\
\text { RR925-3 }\end{array}$ & $\begin{array}{l}20 \\
20\end{array}$ & $\begin{array}{l}3 \\
3\end{array}$ & $\begin{array}{l}3 \\
3\end{array}$ & $\begin{array}{l}50 \\
50\end{array}$ & $\begin{array}{l}10 \\
20\end{array}$ & $\begin{array}{l}70 \\
70\end{array}$ & $\begin{array}{l}300 \\
300\end{array}$ & $\begin{array}{l}50 \\
50\end{array}$ & $\begin{array}{l}\text { ND } \\
\text { ND }\end{array}$ \\
\hline FC-436 & $\begin{array}{l}\text { R901-2 } \\
\text { R919..4 }\end{array}$ & $\begin{array}{l}20 \\
20\end{array}$ & $\begin{array}{l}3 \\
3\end{array}$ & $\begin{array}{r}10 \\
5\end{array}$ & $\begin{array}{l}70 \\
50\end{array}$ & $\begin{array}{l}15 \\
10\end{array}$ & $\begin{array}{r}80 \\
100\end{array}$ & $\begin{array}{l}500 \\
400\end{array}$ & $\begin{array}{l}150 \\
100\end{array}$ & $\begin{array}{l}\text { ND } \\
\text { ND }\end{array}$ \\
\hline FC-437 & $\begin{array}{l}\text { MER41-1 } \\
\text { MER41- }\end{array}$ & $\begin{array}{l}20 \\
10\end{array}$ & $\begin{array}{l}3 \\
3\end{array}$ & $\begin{array}{l}3 \\
3\end{array}$ & $\begin{array}{l}50 \\
50\end{array}$ & $\begin{array}{l}10 \\
10\end{array}$ & $\begin{array}{l}\mathbf{5 0} \\
\mathbf{5 0}\end{array}$ & $\begin{array}{l}400 \\
400\end{array}$ & $\begin{array}{r}100 \\
60\end{array}$ & $\begin{array}{l}\text { ND } \\
\text { ND }\end{array}$ \\
\hline FC- -473 & $\begin{array}{l}\text { S39-4 } \\
\text { S14-? }\end{array}$ & $\begin{array}{r}5 \\
20\end{array}$ & $\begin{array}{l}\text { ND } \\
\text { ND }\end{array}$ & $\begin{array}{l}\text { ND } \\
\text { ND }\end{array}$ & $\begin{array}{l}30 \\
30\end{array}$ & $\begin{array}{l}\text { ND } \\
\text { ND }\end{array}$ & $\begin{array}{l}10 \\
30\end{array}$ & $\begin{array}{l}100 \\
100\end{array}$ & $\begin{array}{l}20 \\
70\end{array}$ & $\begin{array}{l}\text { ND } \\
\text { ND }\end{array}$ \\
\hline FC-501 & $\begin{array}{l}\text { SR52-1 } \\
\text { SR51-6 }\end{array}$ & $\begin{array}{l}5 \\
5\end{array}$ & $\begin{array}{l}\text { ND } \\
\text { ND }\end{array}$ & $\begin{array}{l}\text { ND } \\
\text { ND }\end{array}$ & $\begin{array}{l}30 \\
30\end{array}$ & $\begin{array}{l}\text { ND } \\
\text { ND }\end{array}$ & $\begin{array}{l}10 \\
10\end{array}$ & $\begin{array}{r}100 \\
70\end{array}$ & $\begin{array}{l}20 \\
10\end{array}$ & $\begin{array}{l}\text { ND } \\
\text { ND }\end{array}$ \\
\hline FC-152 & $\begin{array}{l}\text { N512-5 } \\
\text { N5I6-1 }\end{array}$ & $\begin{array}{l}10 \\
10\end{array}$ & $\begin{array}{r}15 \\
5\end{array}$ & $\begin{array}{l}\text { ND } \\
\text { ND }\end{array}$ & $\begin{array}{r}70 \\
100\end{array}$ & $\begin{array}{l}15 \\
15\end{array}$ & $\begin{array}{l}80 \\
60\end{array}$ & $\begin{array}{l}500 \\
400\end{array}$ & $\begin{array}{r}100 \\
80\end{array}$ & $\begin{array}{l}30 \\
30\end{array}$ \\
\hline FC-589 & $\begin{array}{l}\text { T113-2 } \\
\text { T115-2 }\end{array}$ & $\begin{array}{l}4 \\
5\end{array}$ & $\begin{array}{r}\text { ND } \\
7\end{array}$ & $\begin{array}{l}3 \\
3\end{array}$ & $\begin{array}{l}25 \\
50\end{array}$ & $\begin{array}{r}\text { ND } \\
15\end{array}$ & $\begin{array}{l}20 \\
25\end{array}$ & $\begin{array}{l}275 \\
300\end{array}$ & $\begin{array}{l}10 \\
25\end{array}$ & $\begin{array}{l}\text { ND } \\
\text { ND }\end{array}$ \\
\hline FC-590 & $\begin{array}{l}\text { V202-3 } \\
\text { V203-5 }\end{array}$ & $\begin{array}{l}45 \\
60\end{array}$ & $\begin{array}{l}20 \\
20\end{array}$ & $\begin{array}{l}10 \\
10\end{array}$ & $\begin{array}{l}225 \\
200\end{array}$ & $\begin{array}{r}85 \\
125\end{array}$ & $\begin{array}{l}200 \\
200\end{array}$ & $\begin{array}{l}450 \\
550\end{array}$ & $\begin{array}{l}200 \\
225\end{array}$ & $\begin{array}{l}\mathbf{3 0} \\
\mathbf{3 0}\end{array}$ \\
\hline
\end{tabular}

resulted from the ditferential displacement and subsequent push-through of a large fuel fragment.

The $F(-112$ closure veld was also examined metallographically. The two welt cracks (Fig. i3) occurred on the vent cup side of the weld bead and were located just beyond the "spear point" at the end of the overlap weld. Both craciss were intergranulir and had propagated into relatively fine grained sections of the vent cup wali (Fig. 14). Additional grinding reveaied the weld microstructures shown in Fig. 15. Both weid sections contained a large number of equiaxed grains.. and the fractures followed the grain boundaries through the weld bead thickness. Although the metallography did not not indicate whether the fractures resulted from mechanical deformation or the enlargement of preexisting clefects, the fact that toth cracks occurred on the vent cup side of the weld supports the latter theory.

The microstructures of the FC- 165 and $F \mathrm{C}-368$ weld beads are shown in Figs. 16 and 17. The FC- 165 weld failure apparently occurred by fracture along the bound- aries of elongated grains oriented normal to the wail thickness. Similar grain elongation (up to $40 \%$ of the weld thickness) was aiso ohserved in the FC-368 weld.

Cross sections of the FC-364 and FC-368 vent assemblies revealed that both vents were free of intermetallic deposits and vent effusates. Only small quantities of plutonia were observed on the inner surfaces of the vent frits. Excessive grain growth adjacent to the assembly and decontamination cover welds occurred in buth vents (Figs. 18 and 19 ).

Indications of grain bouridary sulfur contamination (from an unknown source) were detected in three iridium wall samples obtained from capsule FC- 165 and in one specimen removed from FC-368. The AES results (Table VIII) also indicated thorium depletion on the clad interiors. Bulk impurity levels were within the expected ranges.

The microstructures of plutonia pellets HF-364 and HF-368 were typical of SRP maierial. No second phase impurities were observed. 


\begin{tabular}{|c|c|c|c|c|c|c|c|c|}
\hline \multicolumn{9}{|c|}{ TABLE XI. SVT Plutonis Analyses } \\
\hline \multirow{2}{*}{$\begin{array}{l}\text { Fueled } \\
\text { Clad }\end{array}$} & \multicolumn{8}{|c|}{ Selected Elements"(ppm) } \\
\hline & $\underline{\mathbf{P}}$ & $\mathrm{Mg}$ & Ca & Al & $\mathbf{S i}$ & $\mathrm{Fe}$ & $\mathrm{Cr}$ & $\mathbf{T i}$ \\
\hline$\overline{\text { FC-112 }}$ & 8 & 3 & 300 & 20 & 15 & 5 & 6 & 15 \\
\hline & 9 & 10 & 50 & 30 & 40 & 10 & 15 & 15 \\
\hline FC. & 5 & 20 & 300 & 20 & 40 & 5 & 7 & 30 \\
\hline FC- & 15 & $\mathbf{1}$ & 25 & 15 & 30 & $\mathbf{N D}^{\mathrm{b}}$ & 10 & 20 \\
\hline FC-391 & 11 & 7 & 250 & 360 & 35 & ND & 9 & 15 \\
\hline FC. & 15 & 7 & 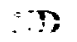 & $\eta^{\prime \prime}$ & $\therefore$ & $\because$ &. & 15 \\
\hline FC-437 & 35 & 7 & 6 & 55 & 15 & 9 & ND & 15 \\
\hline FC-44 & 6 & 7 & 400 & 75 & 5 & ND & 7 & 10 \\
\hline FC-473 & 16 & 5 & 750 & 770 & 40 & 35 & 10 & 35 \\
\hline FC-501 & 14 & 5 & 750 & 145 & 40 & 7 & 7 & 35 \\
\hline FC-591 & 20 & 3 & 750 & ND & 15 & ND & 10 & 35 \\
\hline FC-592 & 4 & 2 & 750 & 85 & 15 & 20 & 8 & 35 \\
\hline FC-15 & 5 & 2 & 750 & 140 & 25 & 25 & 5 & 35 \\
\hline FC & 6 & 1 & 400 & ND & 8 & 10 & 15 & 25 \\
\hline FC-58 & 6 & 1 & 500 & ND & 10 & ND & 7 & 35 \\
\hline FC-590 & 2 & 10 & 750 & 30 & 35 & 15 & 8 & 20 \\
\hline
\end{tabular}

Elements are listed only if they exceed the detectability limit in at least one pellet.

bot detected.

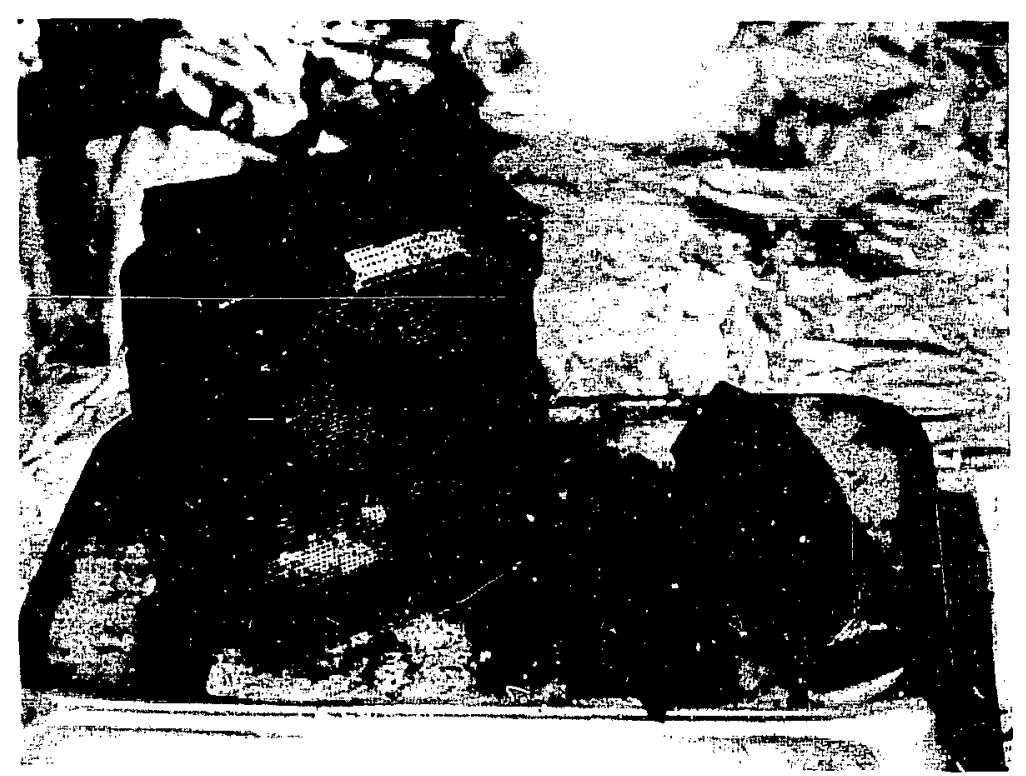

Fig. 4. The SVT-7 aeroshell fractured along the axial contact lines of the leading GIS; $0.4 \mathrm{X}$. 


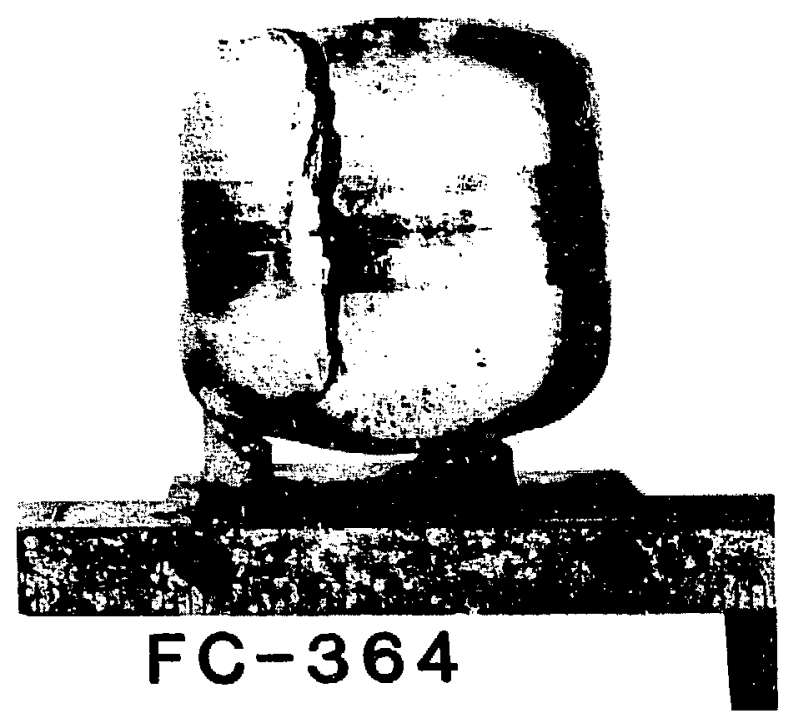

(a)

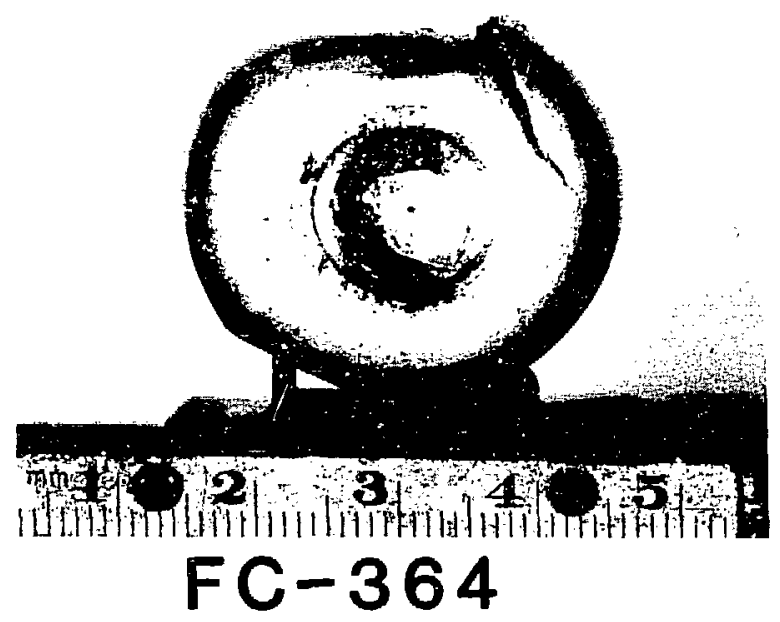

(b)

Fig. 5. A wide axial crack was observed on the impact face of capsule FC-364. (a) Impact face and (b) vent end; both at 1.5X. 


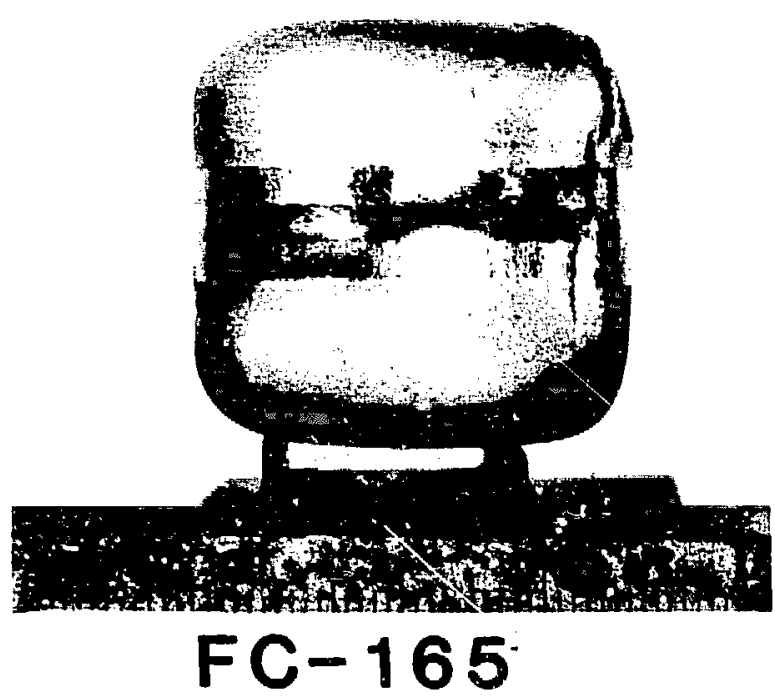

(a)

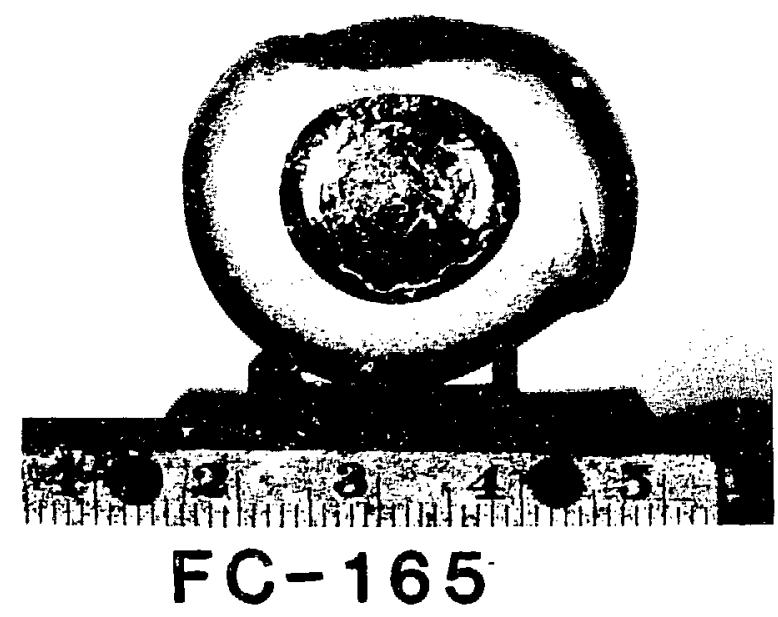

(b)

Fig. 6. The FC-165 impact face was also breacked by a wide axial crack. (a) Impact face and (b) vent end; both at $1.5 \mathrm{X}$. 


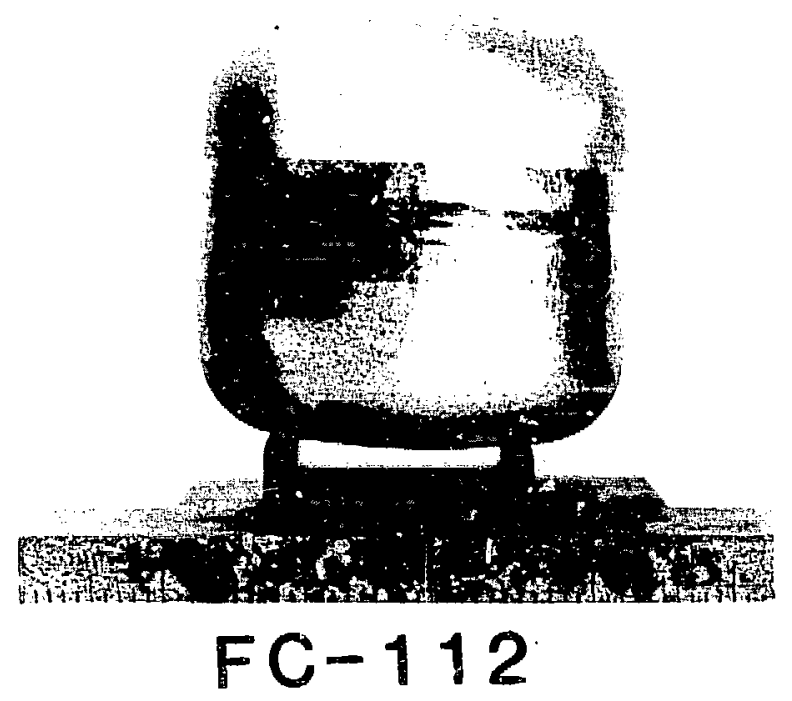

Fig. 7. Two small weld cracks (perpendicular to the weld centerline) were oustrved on the irailing facc of capsule FC-112: $1.5 X$.

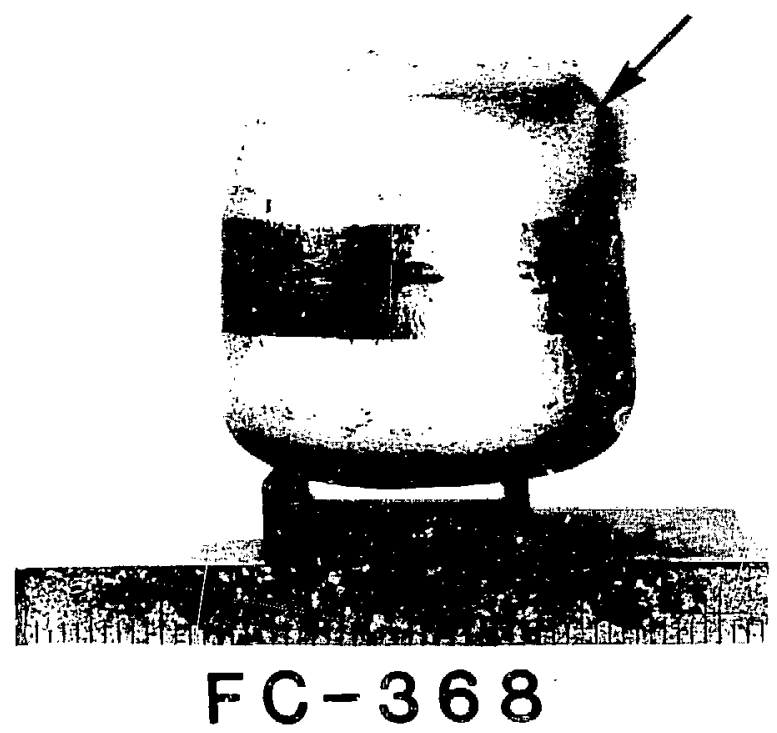

Fig. 8. A small, nonpenetrating crack was observed on the FC-368 vent cup radius; $1.5 \mathrm{X}$. 


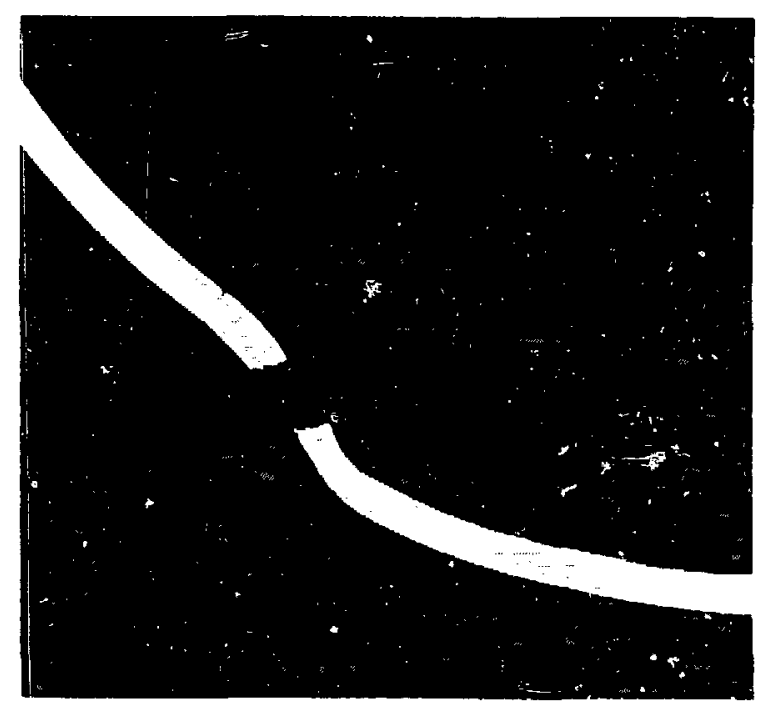

(a)

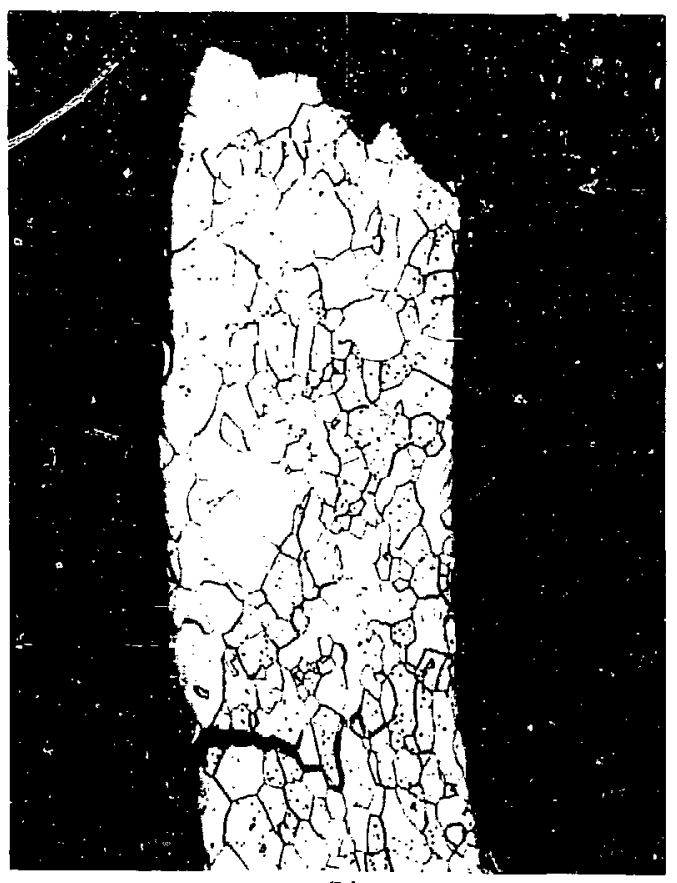

(b)

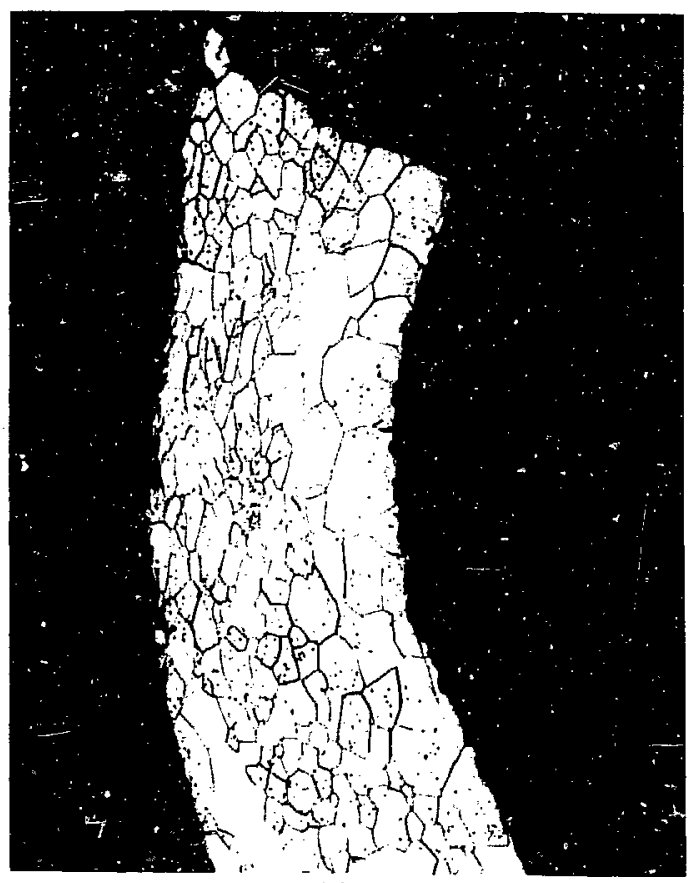

(c)

Fig. 9. Failure in the FC-364 vent cup occurred in a reverse bend region adjacent to the indentation of a large fuel fragment. (a) As polished, 6.5X; (b) and (c) etched, 50X. 


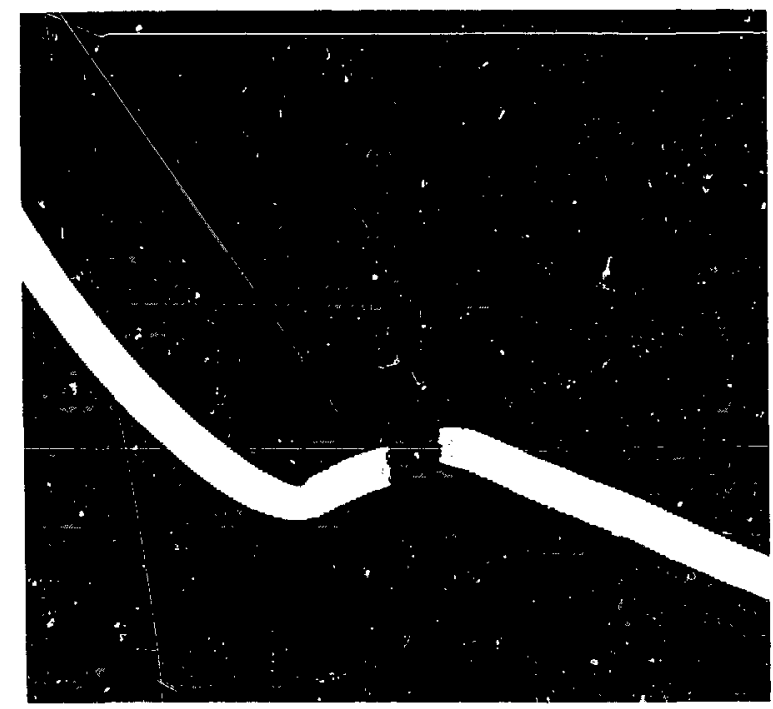

(a)

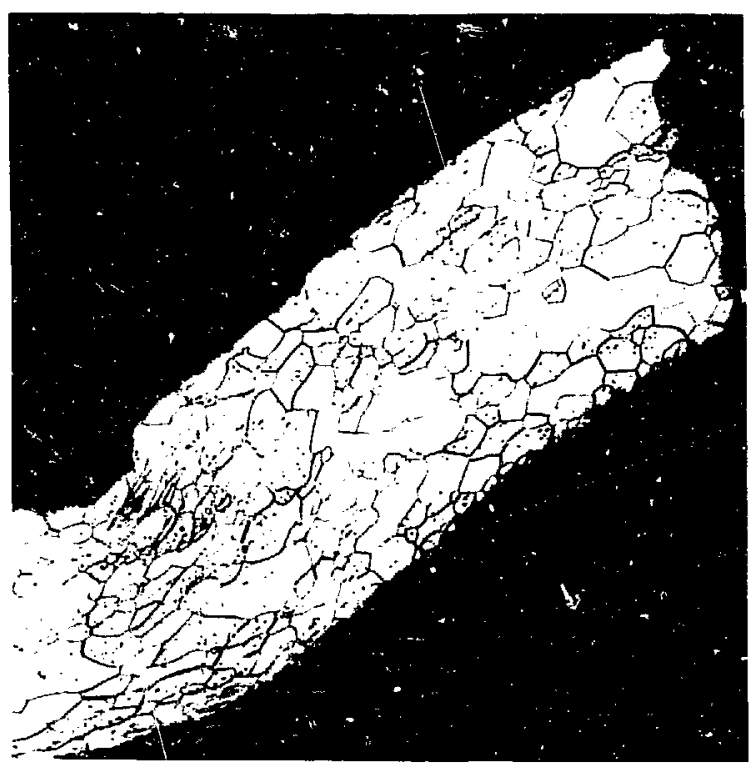

(b)

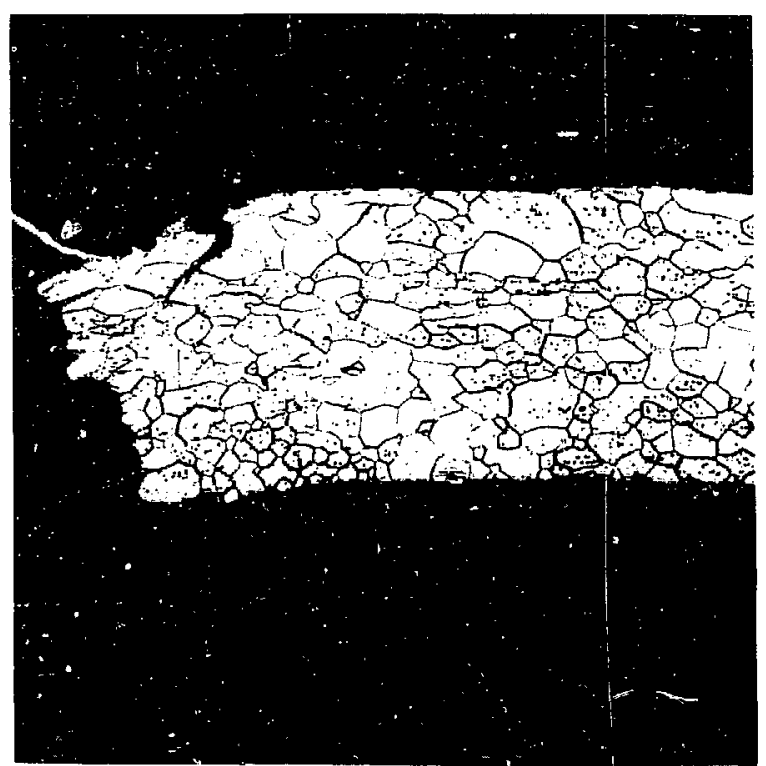

(c)

Fig. 10. Failure in the FC-364 shield cup also occurred in a reverse bend region. (a) As polished, 6.5X; (b) ant (c) etched, $50 \mathrm{x}$. 


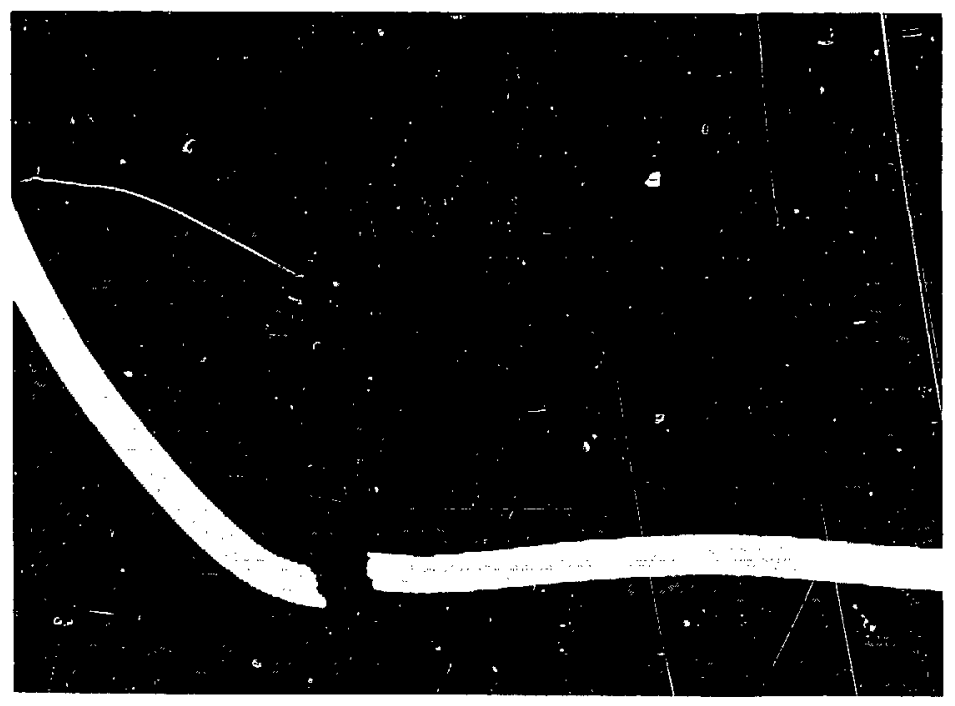

(a)

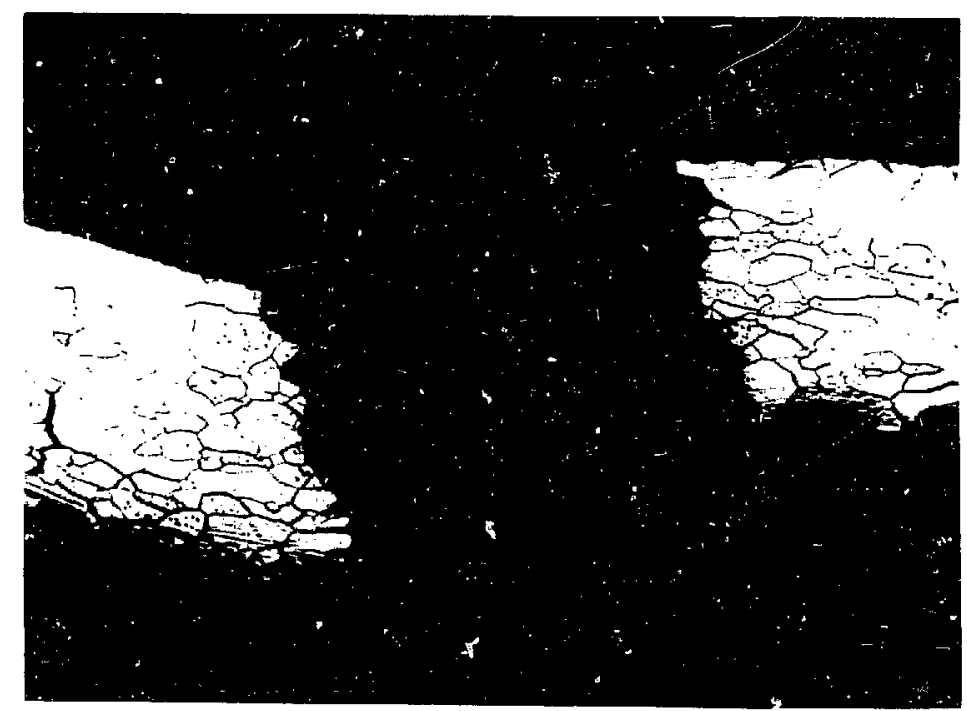

(b)

Fig. 11. The vent cup portion of the FC-165 breach tad a brittle intergranular appearance. (a) As polished, 6.5X; and (b) etched, 50X. 


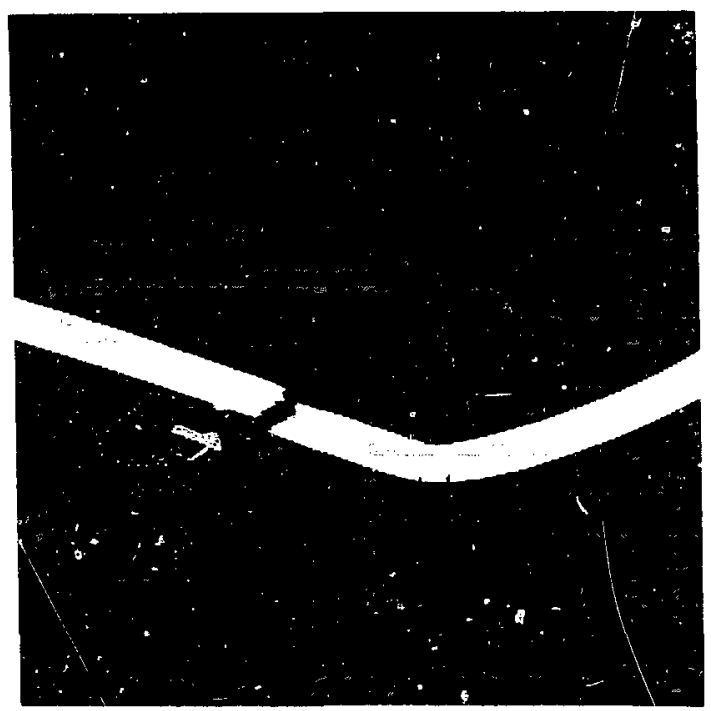

(a)

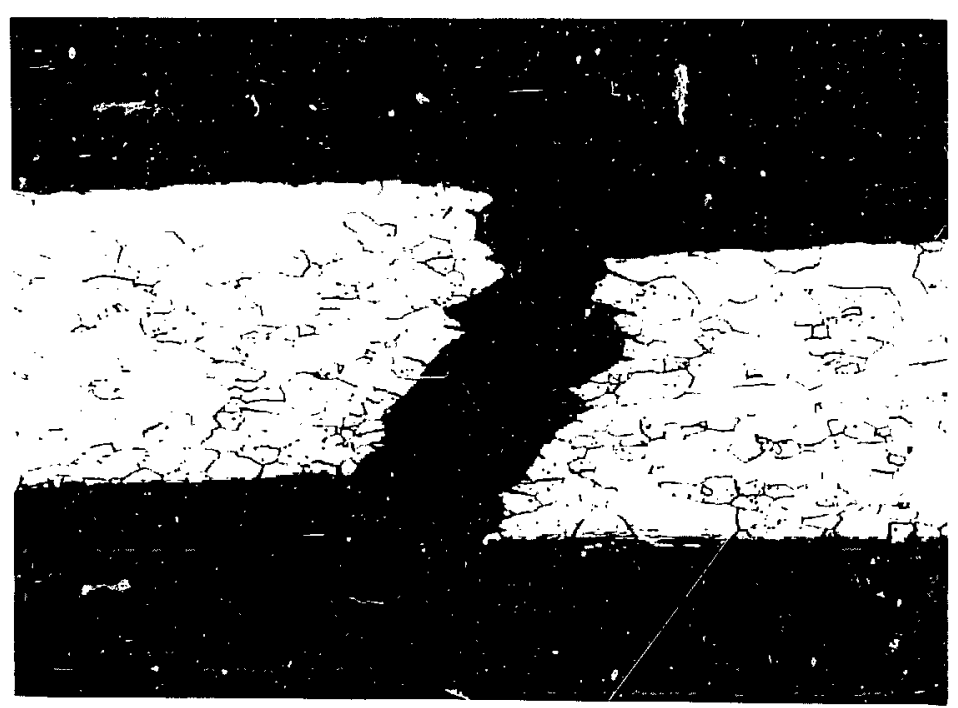

(b)

Fig. 12. The shie d cup portion of the FC-165 breach also had a brittle intergrarular morphology. (a) As polished, 6.5X; and (b) etched, $50 \mathrm{X}$. 


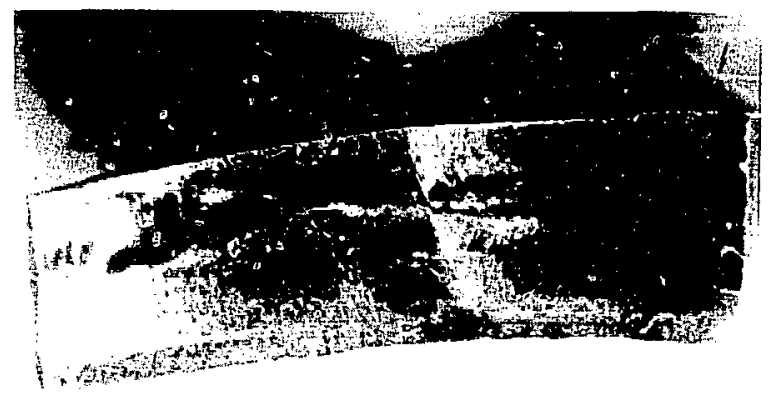

Fig. 13. Cracks in the FC-112 closure weld were located just beyond the "spear point" at the end of the overlap weld; 3.0X.

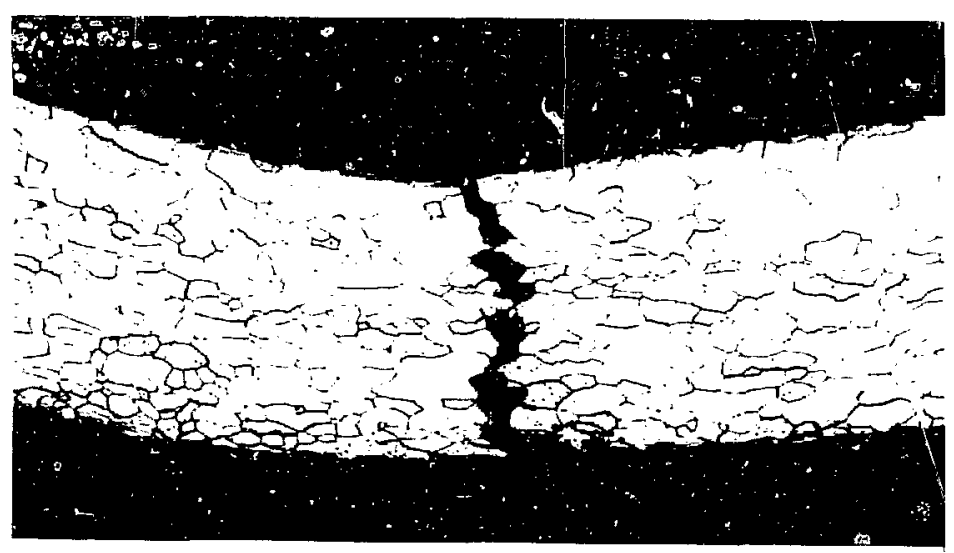

(a)

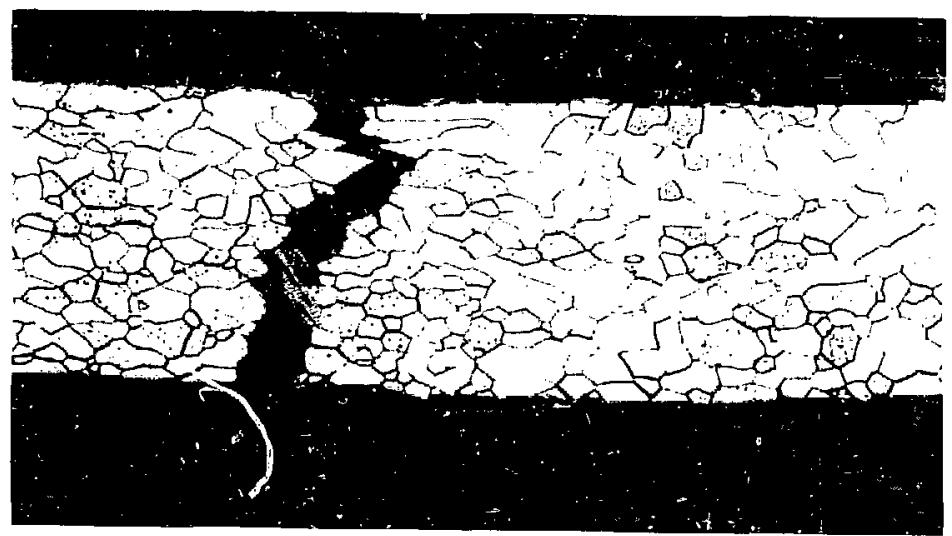

(b)

Fig. 14. The FC-112 weld cracks propagated into the vent cup; (a) and (b) etched, $50 \mathrm{X}$. 


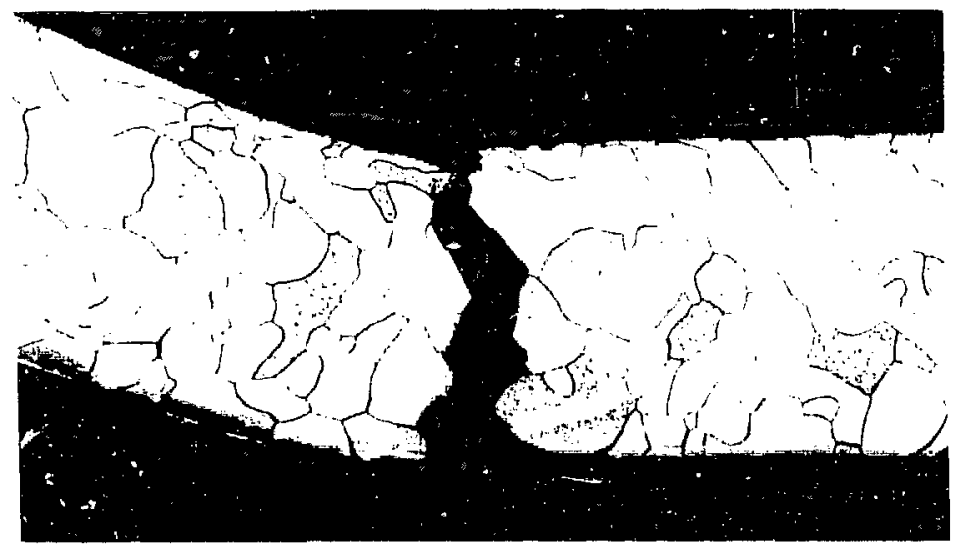

(a)

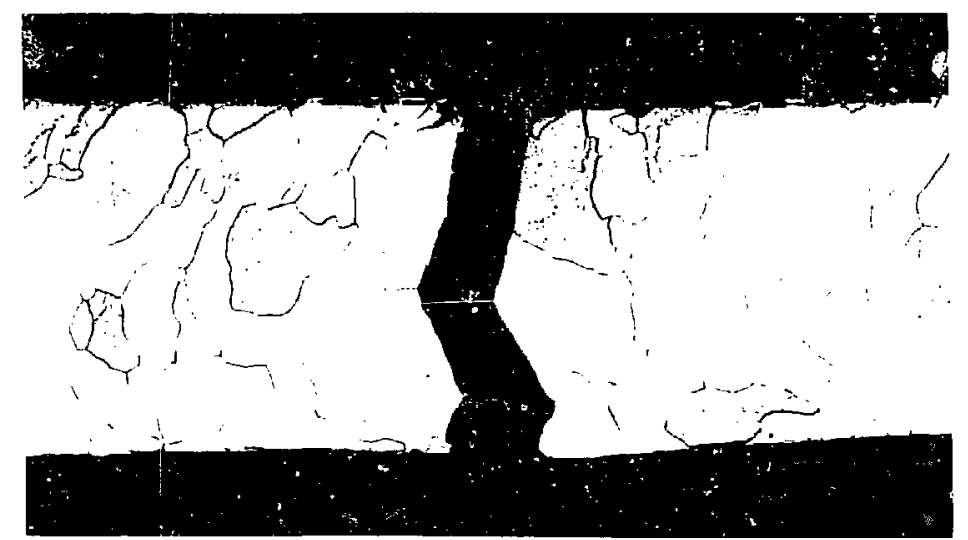

(b)

Fig. 15. The cracked section of the FC-11 2 closure weld had a relatively coarse microstructure composed of large equiaxed grains; (a) and (b) etched, 50X. 


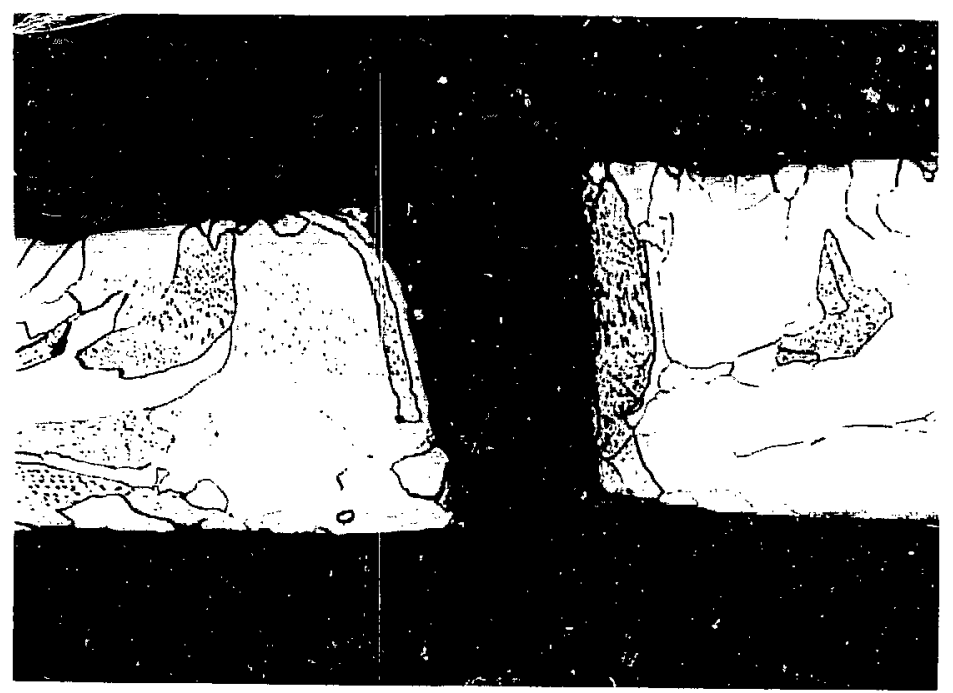

Fig. 16. The FC-165 weld failure occurred in a coarse-grained section; etched, $50 \mathrm{X}$.

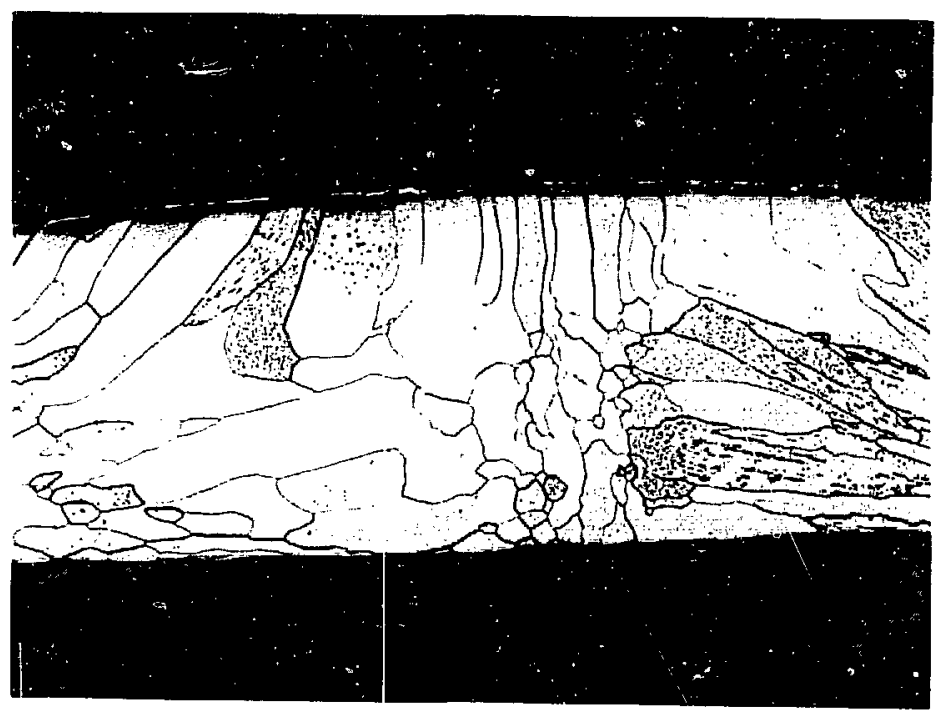

Fig. 17. The FC-368 veld had an unusual microstructure; etched, $50 \mathrm{X}$. 


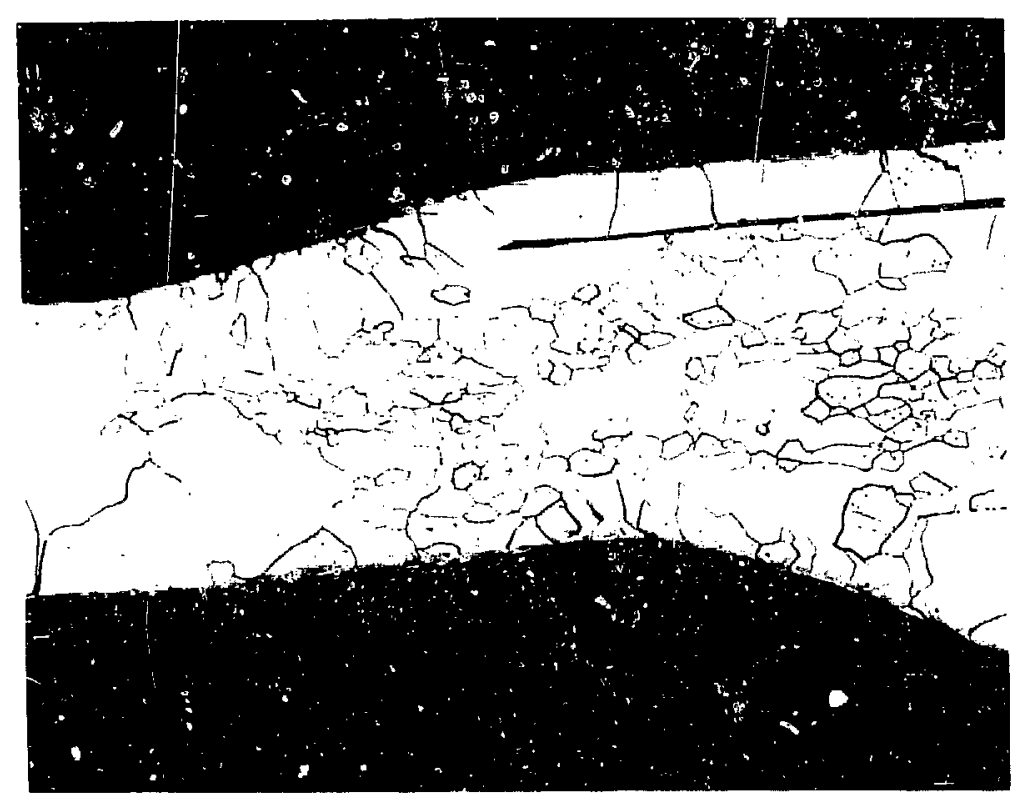

(a)

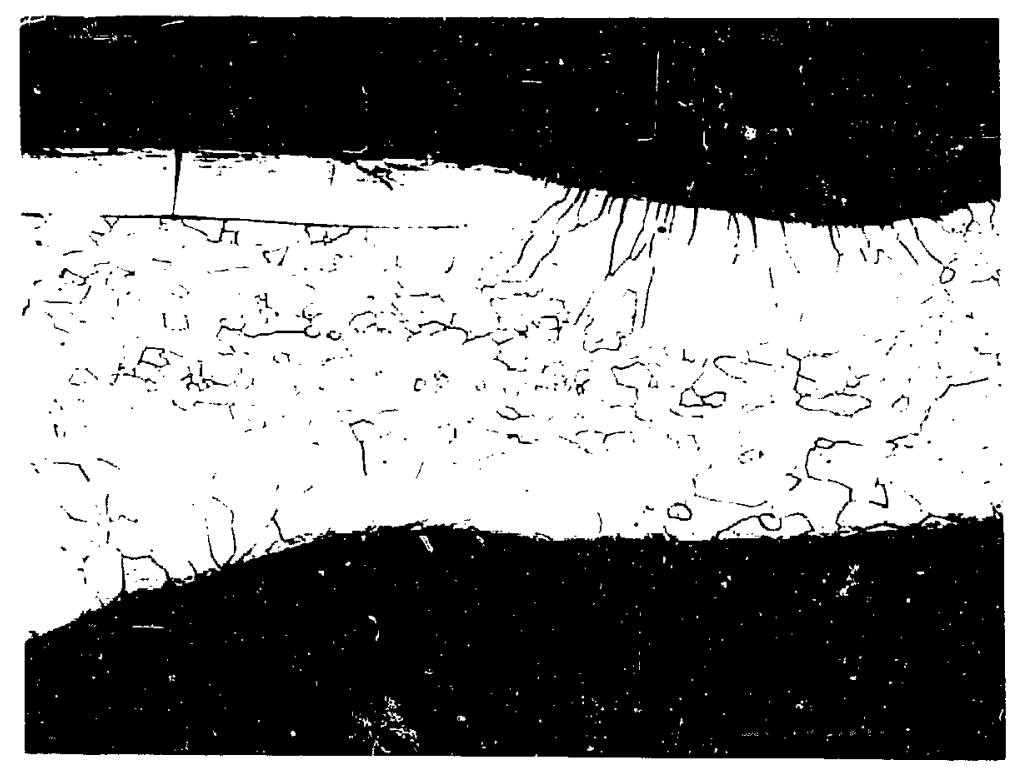

(b)

Fig. 18. Excessive grain growth was observed adjacent to the FC-364 decontamination cover weld; (a) and (b) etched. 50X. 


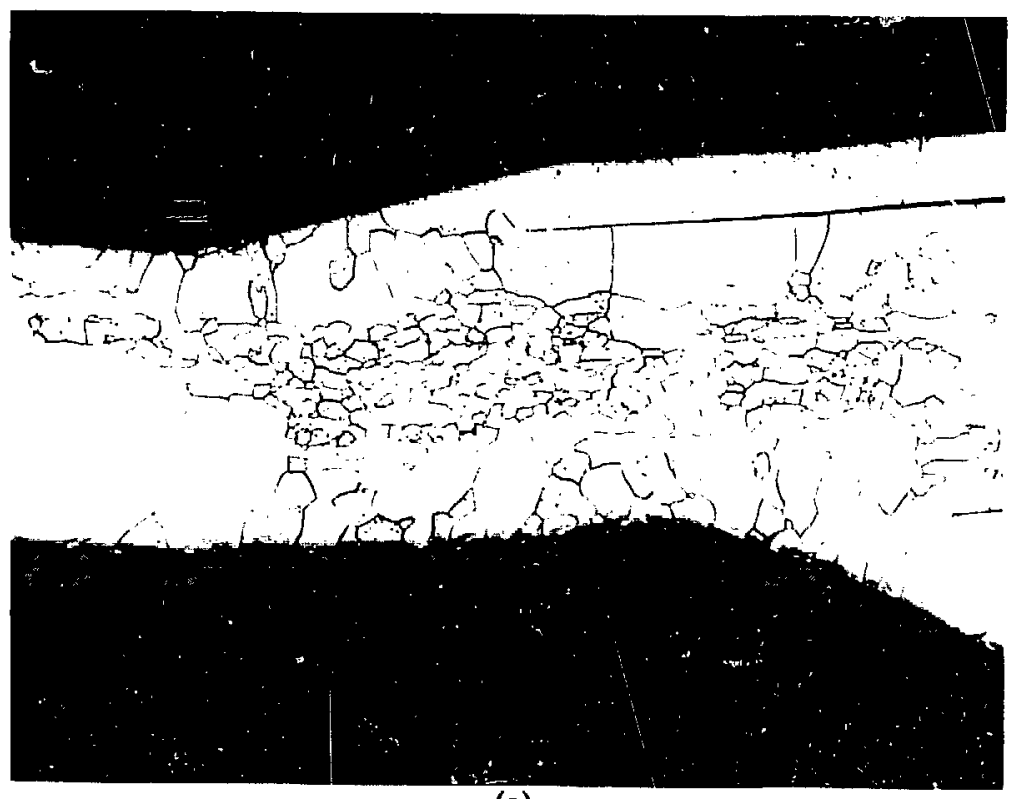

(a)

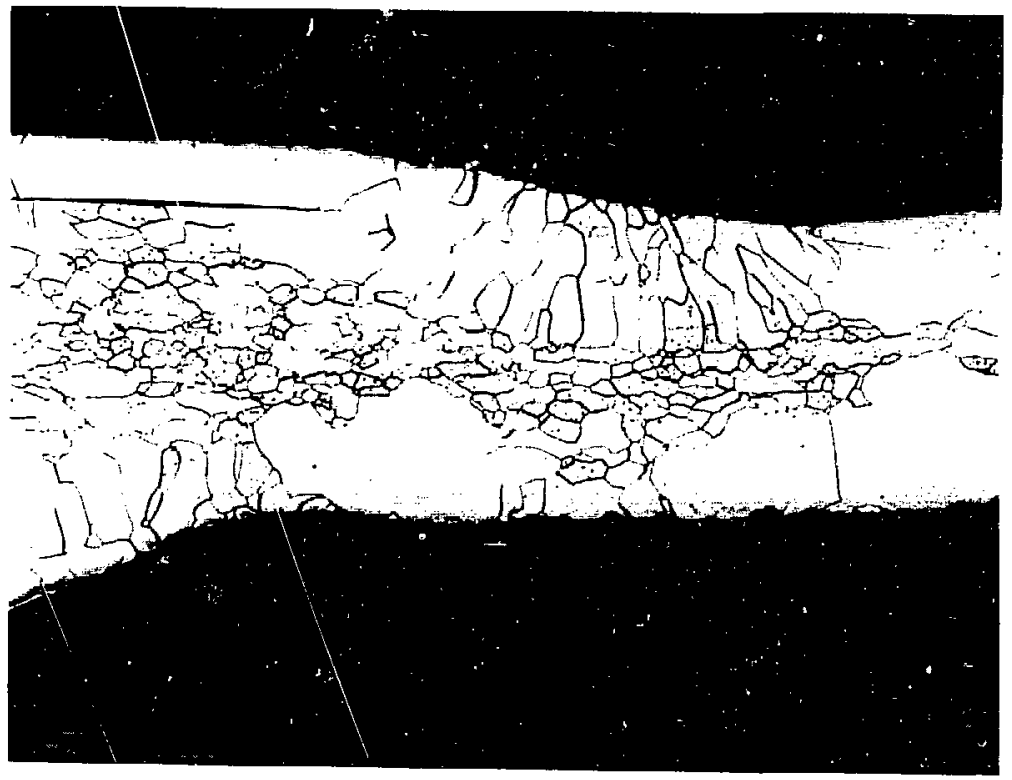

(b)

Fig. 19. Grain coarsening was also observed near the FC-368 deconiamination cover weld; (a) and (b) etched, 50X. 


\section{A. $5 \backslash T-8$}

During the 90- Iy orbitai decay heat treatment, the $\because T$ - s lest module was continuously monitored to deac the helium release; there were no indications of : plugging.

? SVT-8 test module was impacted on a steel target $16 \mathrm{~m} / \mathrm{s}$ and $975^{\circ} \mathrm{C}$. The impact orientation was $15^{\circ}, \beta=0^{\circ}, \gamma=0^{\circ}$. Postimpact examination reit that the aeroshell wails fractured along the axial . Wt lines of the primary GIS (Fig. 20) and that both "hill clost :s were released. Although the GISs $\therefore$ un unestrained, neither left the aeroshell.

ymage to the impact shells was significant but not "in.ual. Irregular axial cracks (up to $75 \%$ of the GIS (ngth) were visible on the impact face of each GilS. Both iis caps had come off, and capsule FC-437 (one of the primary clads) was released into the aerosliell.

Macroscopic examination revealed that the primary clads (FC-436 and FC-437) and one secondary clad (F(-441) were only moderately deformed. Capsule $F(-391$, however, was badly breached (Fig. 21). The FC-39l closure weld failed over a $120^{\circ}$ arc, with the crack center $90^{\circ}$ to the impact face. The crack size $(30.0$ $\mathrm{mm} \times 1.8 \mathrm{~mm}$ ) permitted excessive clad deformation and generated secondary cracks in the vent and weld shield cups (Fig. 22). The weld shield band remained in place and minimized fuel release.

Although the FC-391 closure weld had an SRP/NDE rating of 9.8, weld quality was poor. Metallographic examination revealed slight misalignment of the vent and weld shield cups (Fig. 23) and significant porosity in weld remnants on either side or he breaching crack (Fig. 24). In addition, two large voids (with maximum diameters of approximately $0.33 \mathrm{~mm}$ ) were observed in a face on the weld section containing the terminus of the breaching crack (Fig. 25).

Examination of the FC-436 and FC-437 closure welds did not reveal any unusual features. Both welds had iypical microstructures and were free of observable defects

The iridium cups used in capsules FC-391, FC-436, and FC-437 had acceptable microstructures. Although all six cups contained isolated areas of abnormal grain growth, the microstructures were generally fine grained, with cup grain sizes ranging from 12 to 15 grains/0.635$\mathrm{mm}$ nominal wall thickness. No significant chemical or microstructural anomalies were observed in any of the iridium cups.

Examination of the axial crack on the FC-391 shield cup (Fig. 26) revealed the same intergranular morphology observed in previous iridium fractures. There was no evidence to inwicate that the crack resulted from anything other than excessive clad deformation permitted by the weld failure.
The FC-436 and FC-437 capsule vents did not contain any mechanical defects and were relatively undeformed. Although ihe vent frits were clear, glassy deposits were observed on the vent exteriors and center holes (Fig. 27). The deposits inter ranularly attacked the iridium (Figs. 28 and 29) and promoted excessive grain growth. Microprobe analyses indicated that the deposils contained iridium, oxygen, silicon, and tungsten.

Metallographic examination of the FC-437 capsule vent revealed abnormally large iridium grains (un to 2 grains $/ 0.635-\mathrm{mm}$ nominal wall thickness) near the vent cover we!d (Fig. 30). The grain coarsening was similar to that observed on the SVT-7 vent cover welds. The microstructure of the FC -436 vent cover weld was not affected.

All of the SVT-8 fue! pellets had typical microstructures; no second phase impurities were observed in any pellet. Although pellets HF-391 and HF-44! had elevated calcium contents, the slight variations in chemistry apparently did not affect the impact responses. The pelleis all fractured in a brittle, entirely random manner.

\section{SVT-9}

During the orbital decay aging heat treatment $(90$ days), the test module was continuously monitored to determine helium release; there were no indications of vent plugging.

After aging and reentry heat treatments, the module was impacted against a steel target at $54.6 \mathrm{~m} / \mathrm{s}$ and

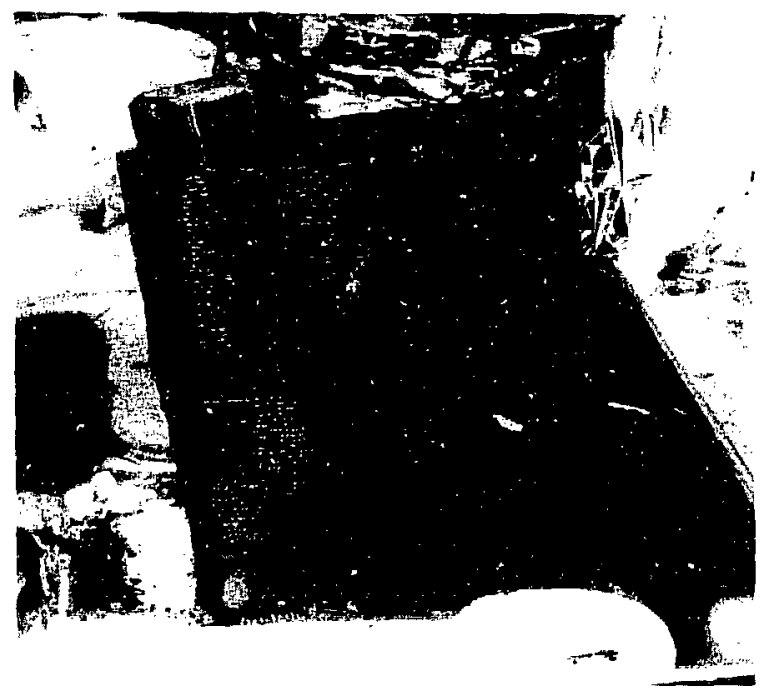

Fig. 20. The walls of the SVT-8 aeroshell fractured alung the axial contact lines of the primary GIS; $0.4 X$. 


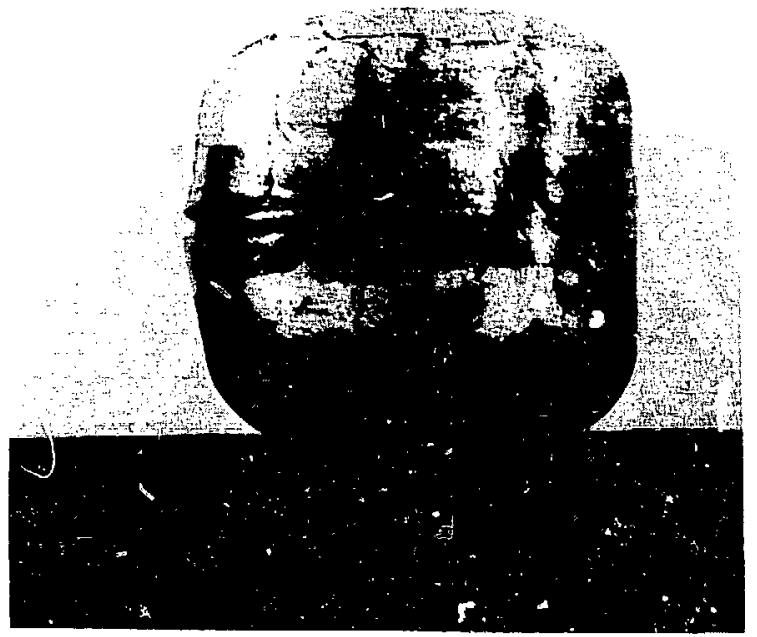

(a)

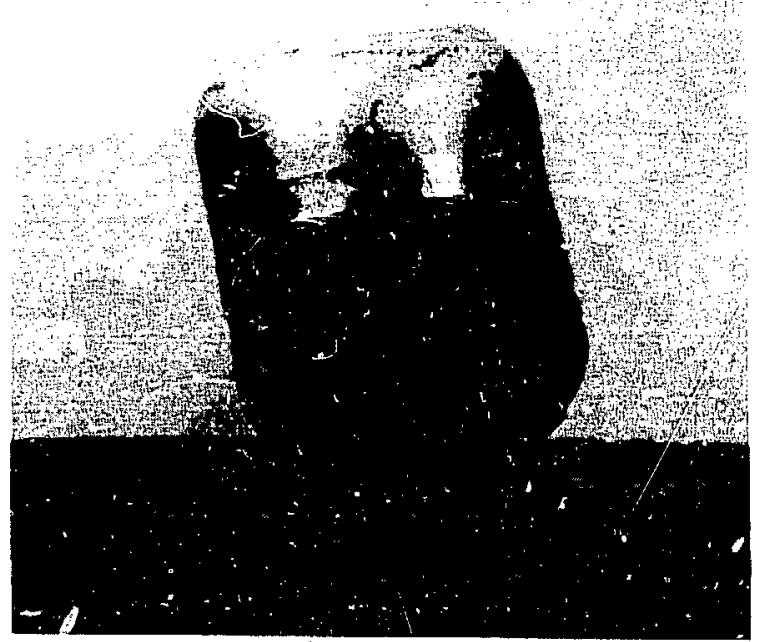

(b)

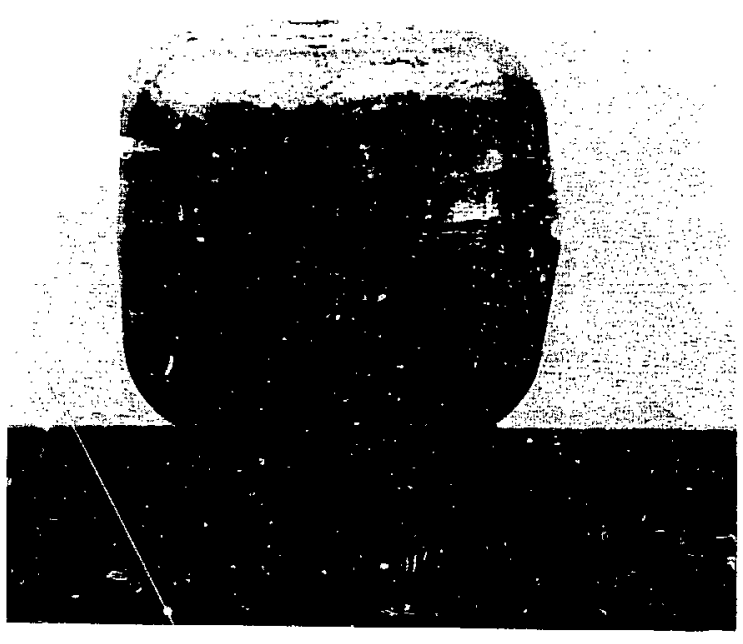

(c)

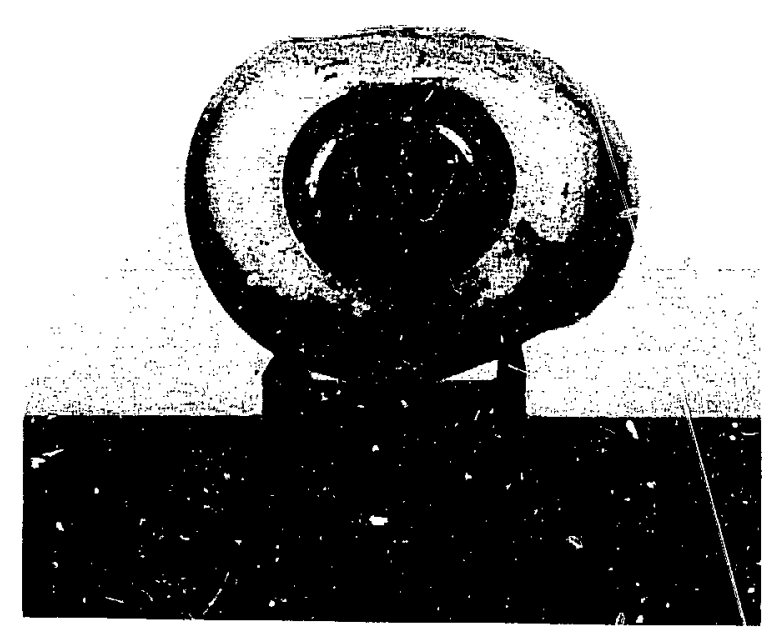

(d)

Fig. 21. The FC-39l closure weld failed over a $120^{\circ}$ arc. (a) Impact face, (b) profile, (c) trailing face, and (d) vent end; all at $1.5 \mathrm{X}$. 


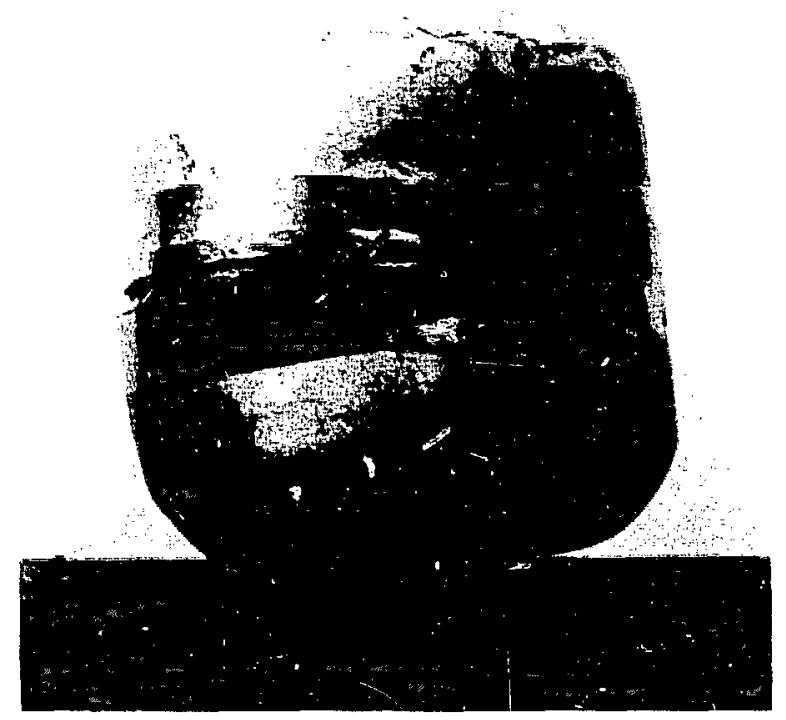

(a)

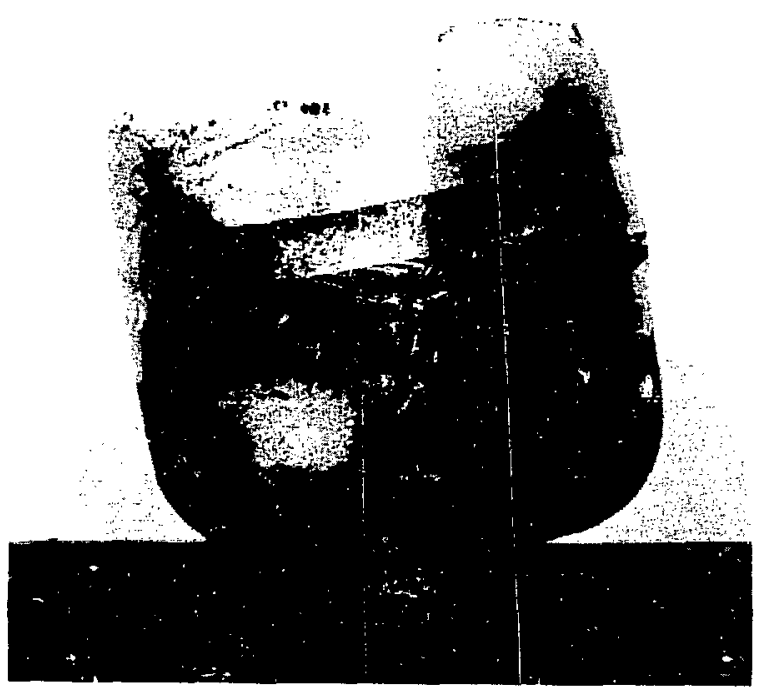

(b)

Fig. 22. The deformation produced by the FC-391 weld failure propagated cracks into the vent and weld shield cups. (a) Small crack in the vent cup and (b) axial crack in the shield cup; both at $2.0 \mathrm{X}$. 


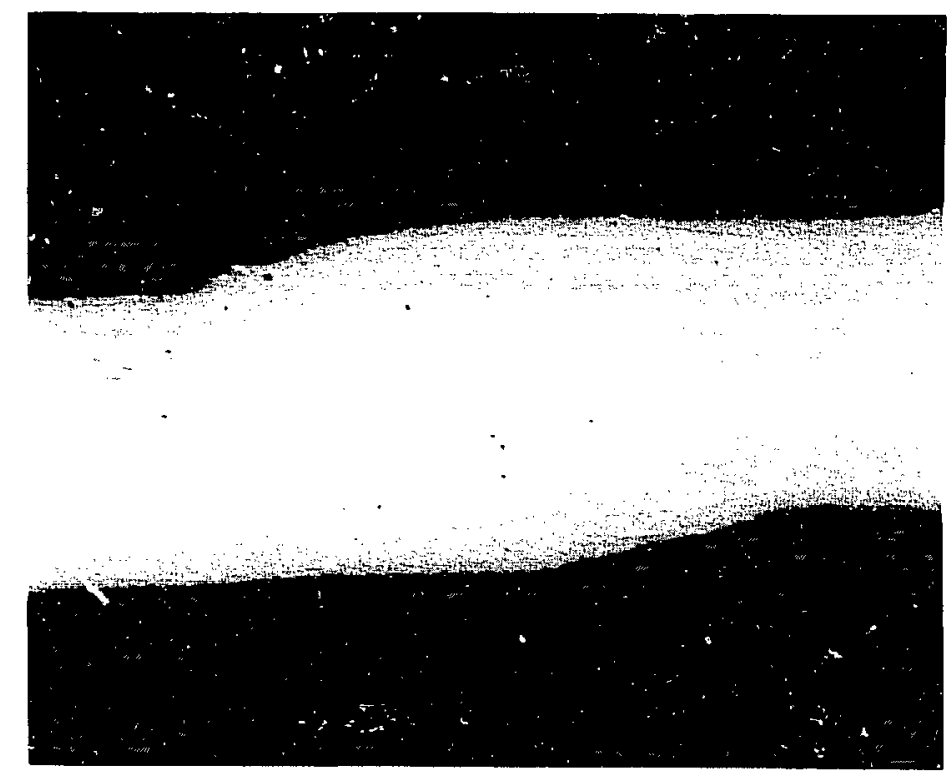

(a)

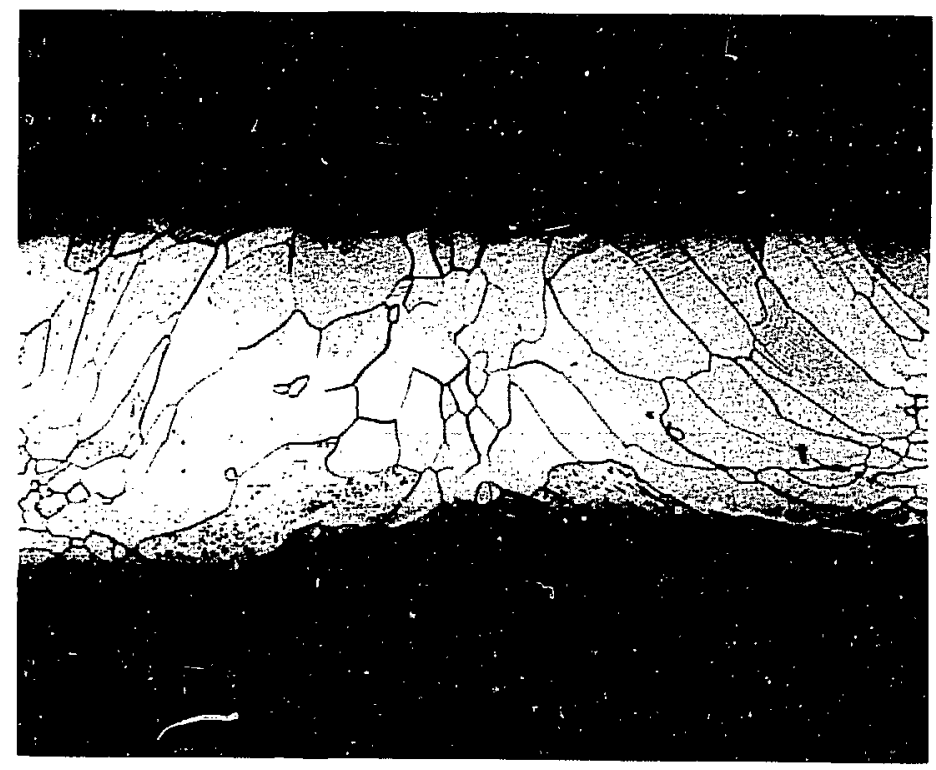

(b)

Fig. 23. The FC-391 vent and weld shield cups were slightly misaligned. (a) As polished and (b) etched; both at 50X. 


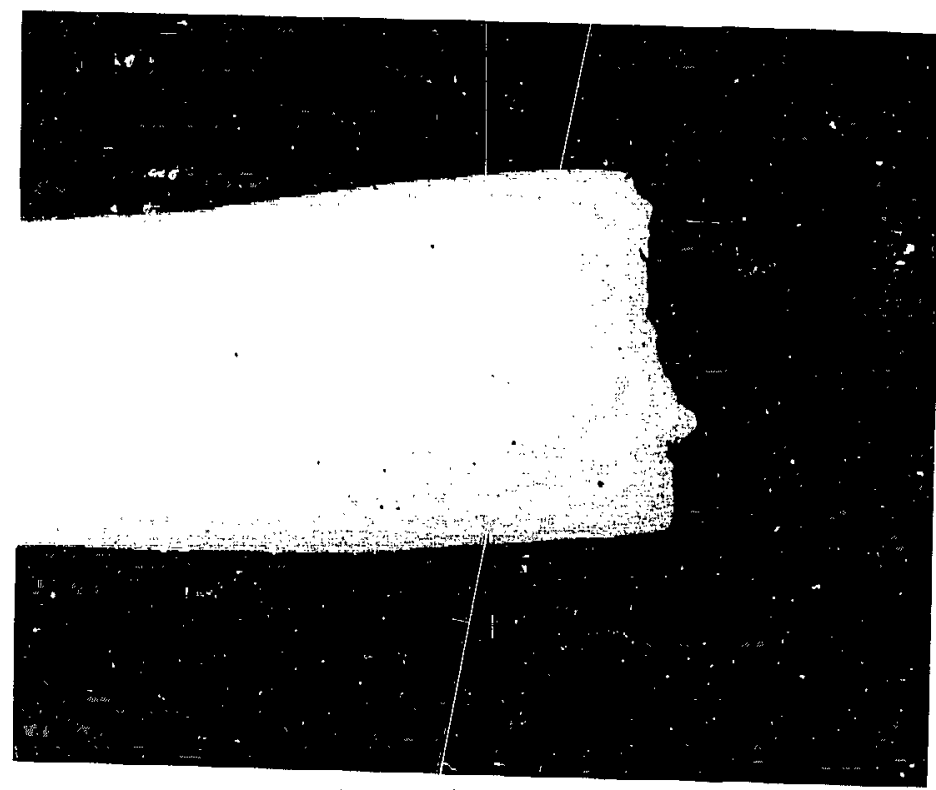

(a)

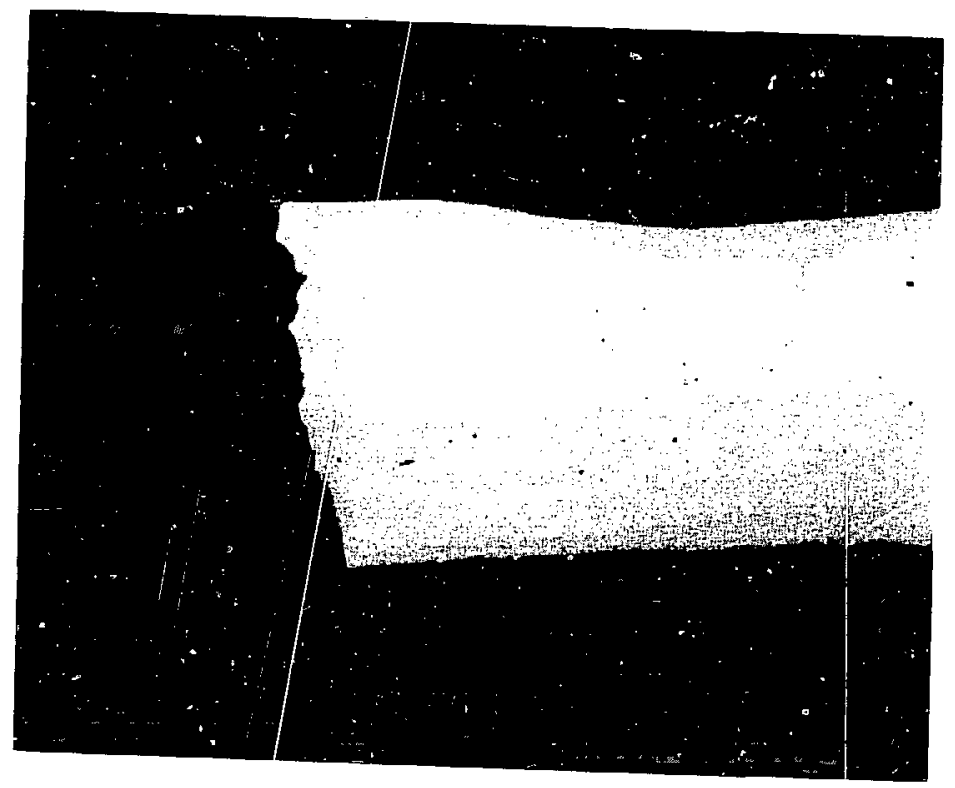

(b)

Fig. 24. Rem!nants oi: either side of the FC-391 "veld failure were slightly porous. (a) Vent cup side and (b) shield cup side;
ioth at $50 \mathrm{X}$ 


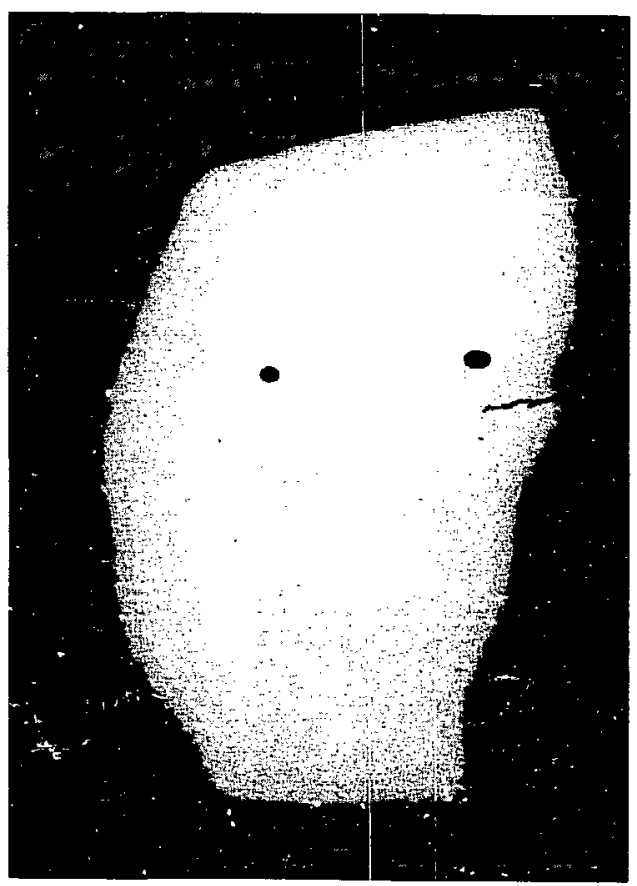

(a)

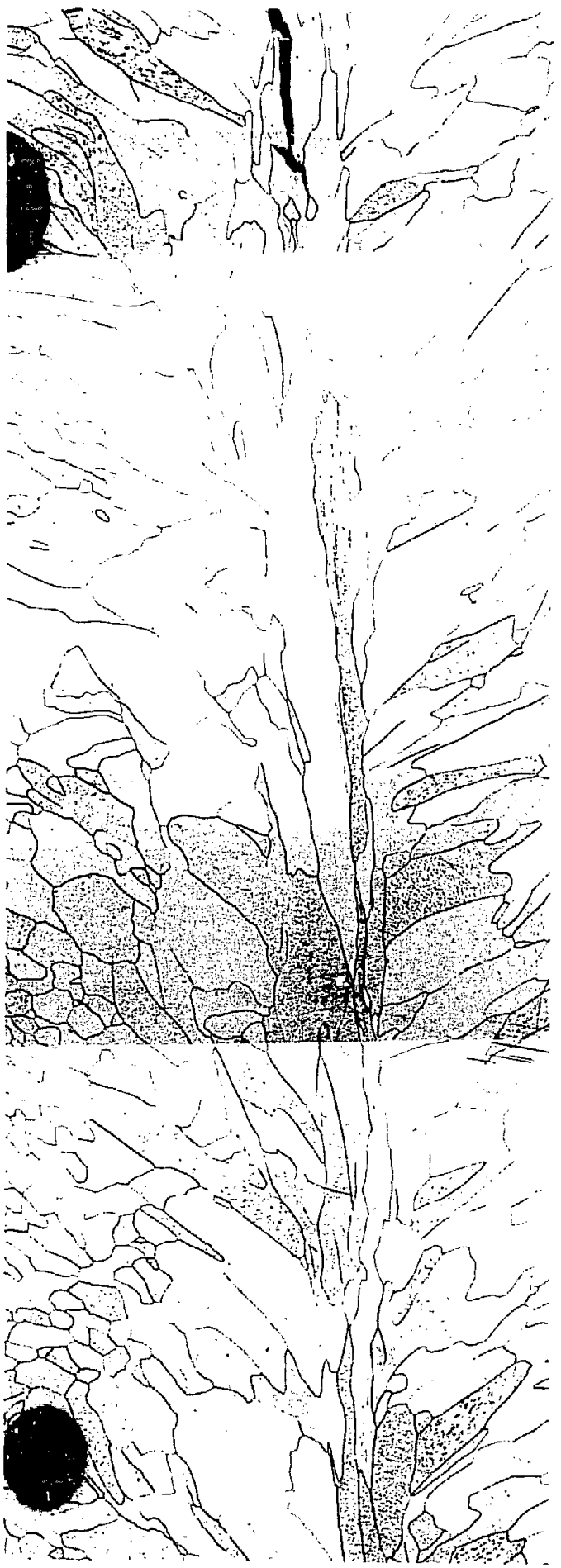

(b)

Fig. 25. Large voids were observed in a face-on section of the FC-391 closure weld. (a) As polished, 7.0X; and (b) etched, $50 \mathrm{X}$. 


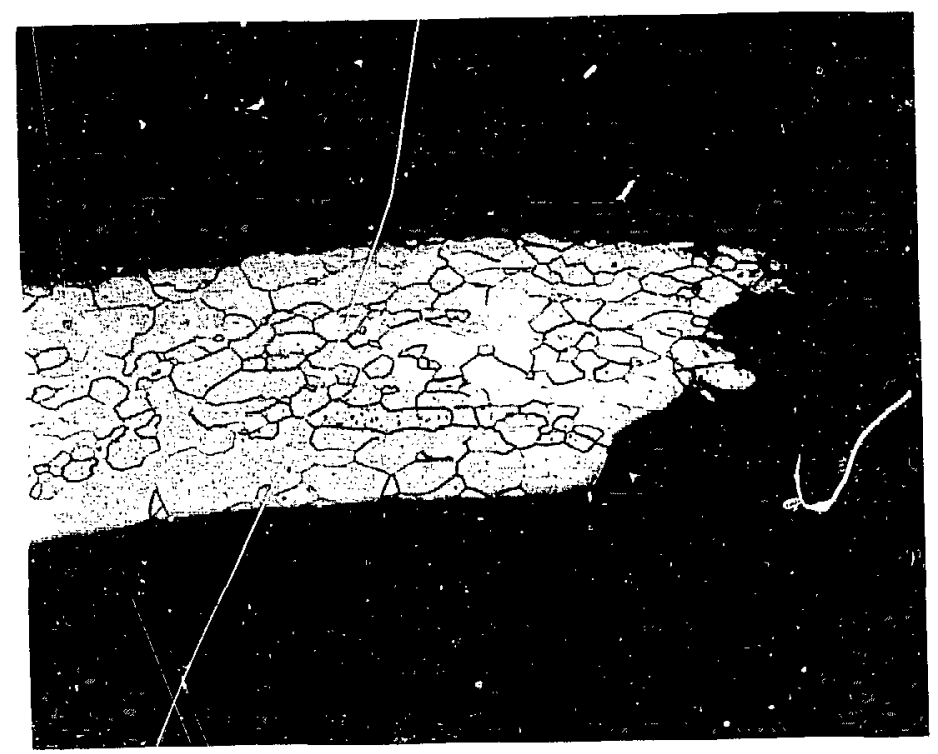

(a)

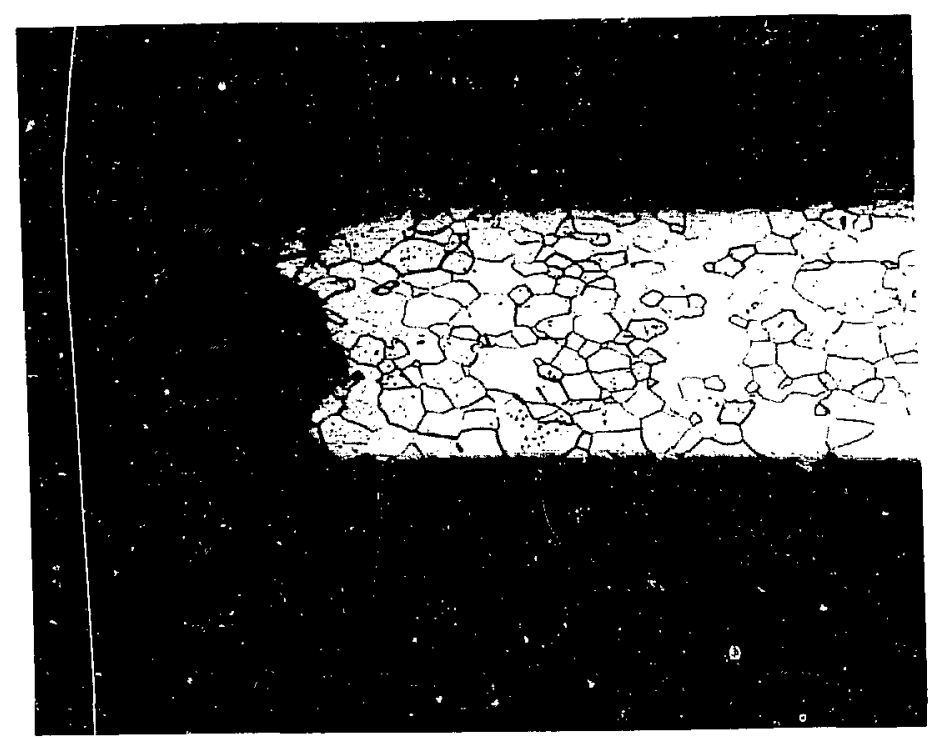

(b)

Fig. 26. The axial crack in the FC-391 shield cup had a brittle intergranular appearance. Opposite crack sides (a) and (b), etched, 50X. 


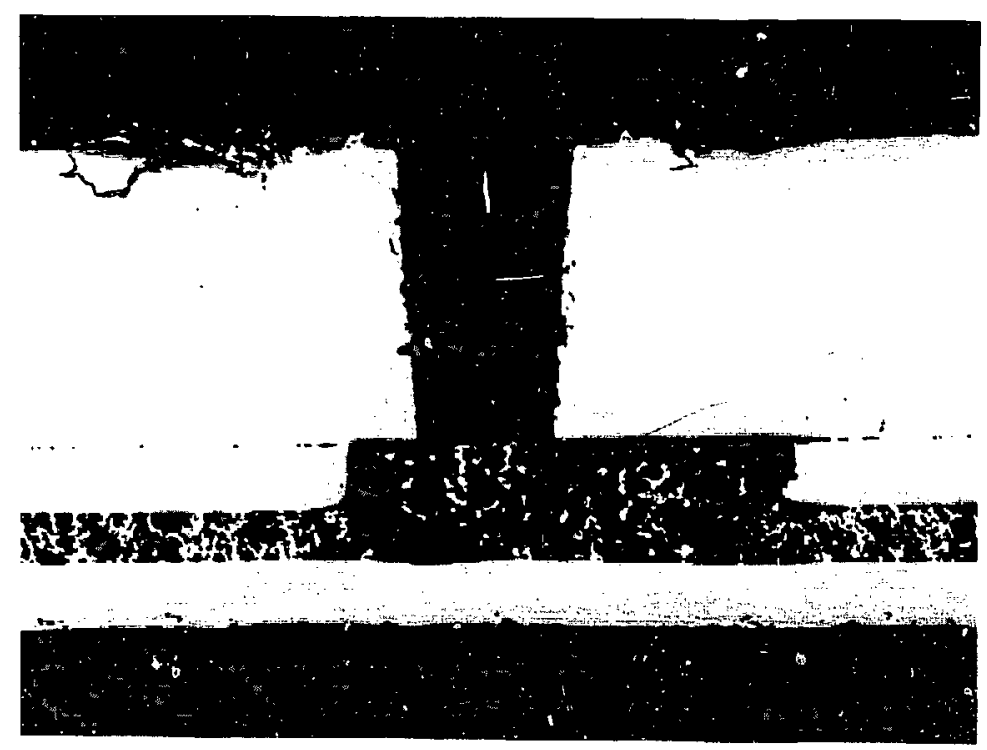

Fig. 27. Glassy deposits were observed on the FC-436 and FC-437 vent exteriors. The FC-436 vent is shown here as polished, 50X.

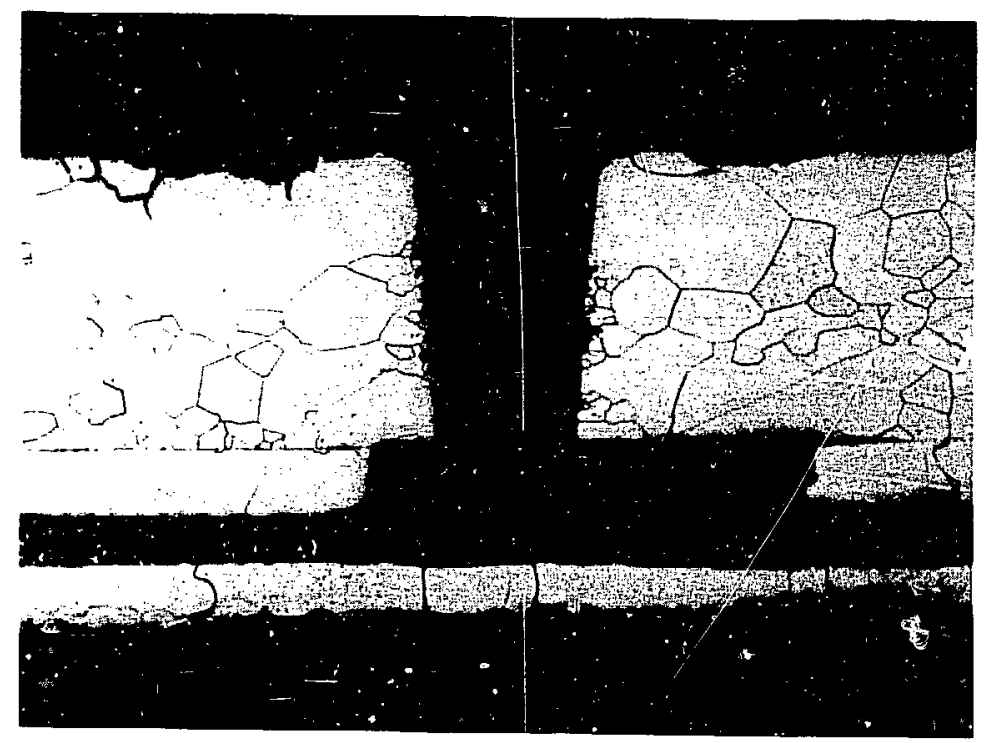

Fig. 28. The deposits on the FC-436 vent intergranularly attacked the iridium; etched, 50X. 


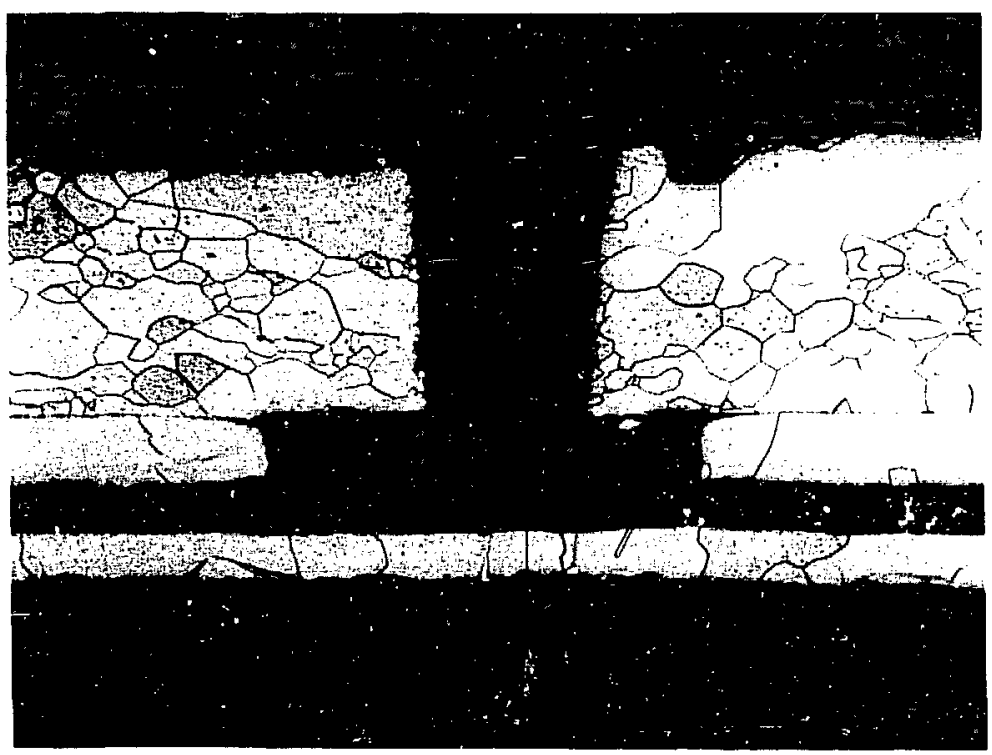

Fig. 29. Deposits on the FC-437 vent had a similar appearance, and they apparently promoted excessive grain growth; etched, $50 \mathrm{X}$. 


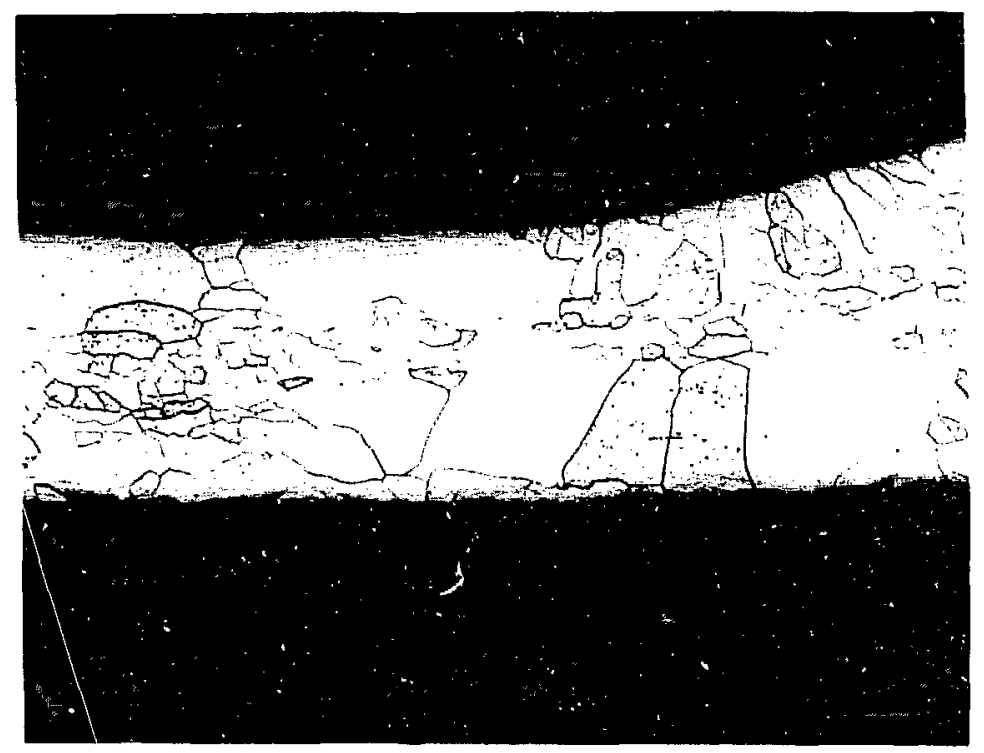

(a)

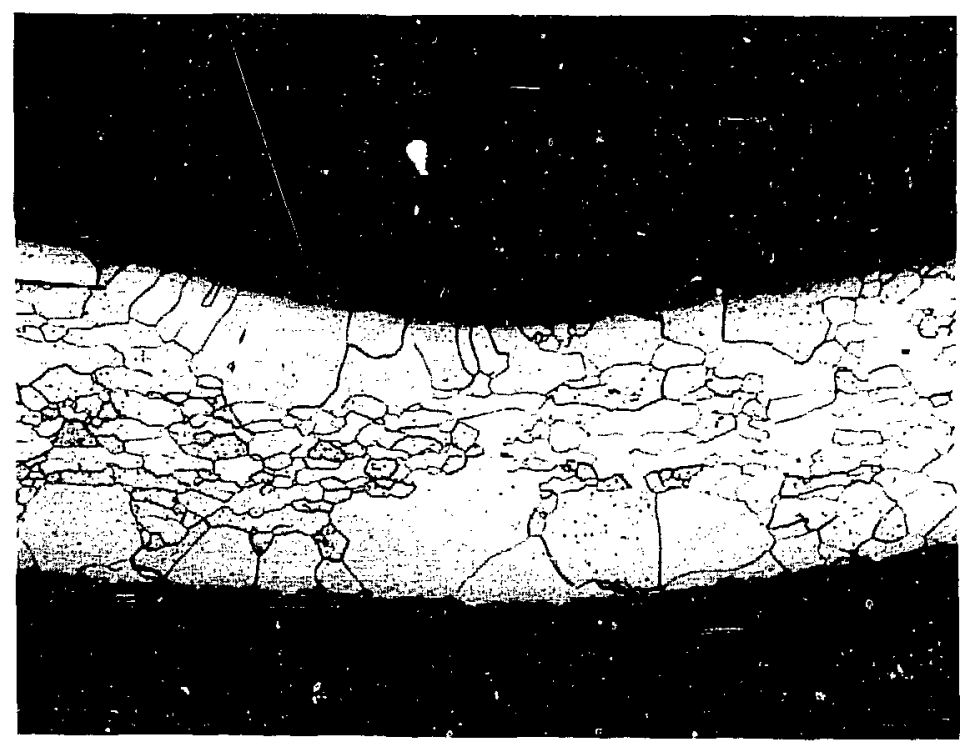

(b)

Fig. 30. Excessive grain growth was observed adjacent to the FC-437 vent decontamination cover weld. (a) Impact face side and (b) opposite side; both at SOX. 
$975^{\circ} \mathrm{C}$. The impact orientation was $\alpha=30^{\circ}, \beta=0^{\circ}$, $y=0^{\circ}$. Because of a loading error, the secondary impact assembly (containing capsules FC-473 and FC-501) was placed in the lead (primary) position. For purposes it the postmortem examination, capsules FC-473 and if - $\$ 01$ were treated as "primary" clads.

terushell damage was greater than that observed in ther $u=\beta=\gamma=0^{\circ}$ or $\alpha=15^{\circ}, \beta=\gamma=0^{\circ} \mathrm{im}$ Dar $\cdots$ A photograph of the impacted aeroshell is shown ir. Hig. 31. Two fragments broke away from the leading musd face of the aeroshell, and the aeroshell body liaciured on the trailing broad face, roughly parallel to the axis of the secondary impact shell. Although the impat faces of both GISs were cracked, with fractures running nearly the GIS length, the leading impact shell was significantly more deformed than the trailing GIS (Fig. 32).

There were no failures in any of the fueled clads. Capsule deformation was generally symmetric, and localized deformation of the iridium was mild. Figure 33 illustrates the deformation of fueled clads from the closure ends of the both impact shells. Capsule FC-591 was slightly more deformed than the other clads.

The microstructures of the FC-473 and FC-501 iridium cups were characterized by moderate grain growth on $25-30 \%$ of the inner wall thickness. However, large grains were also present in several locations on the FC-473 vent cup exterior (Fig. 34). Isolated areas of abnormal grain growth were also seen on the exterior of the FC-501 vent $\mathrm{Cu}_{2}(\mathrm{Fig} .35)$ and in both weld shield cups (Fig. 36). One section of the FC-501 weld shicld cup contained a single grain that occupied $75 \%$ of the total wall thickness (Fig. 36b).

AES analyses indicated thorium depletion on the iridium cup interiors. Sulfur segregation to the grain boundaries was also found in all but one sample. Sulfur concentrations were low in the FC. 473 cups $\left(S_{150 /} I R_{229}\right.$ ratio $<0.15$ ) but appeared to be significantly higher in both of the FC-501 cups. In two sections removed from the FC-50l cups, the sulfur peaks overloaded the instrument.

Spectrographic analyses of the iridium indicated low concentrations of iron (approximately $100 \mathrm{ppm}$ ) and aluminum cortents ( $30 \mathrm{ppm}$ ) significantly lower than those observed in previous test clads.

Examination of cross sections from the FC -473 and FC-501 vents revealed that the vent orifices contained 110 intermetallic deposits. Only small quantities of plutonia were observed at the entrances to the vent filter elements. Abnormal grain growth occurred adjacent to the vent assembly and decontamination cover welds (Figs. 37 and 38).

The microstructures of the plutonia fuel pellets were typical. Ail of the pellets had calcium contents of 750 ppm and HF-373 had an aluminum content of $770 \mathrm{ppm}$. Although the aluminum and calcium contents exceeded the SRP guideline limits (150 and 300 ppm, respectively), there were no apparent differences between the in 1 pact responses of the SVT-9 fuel pellets and those of pellets used in previous SVT impacts.

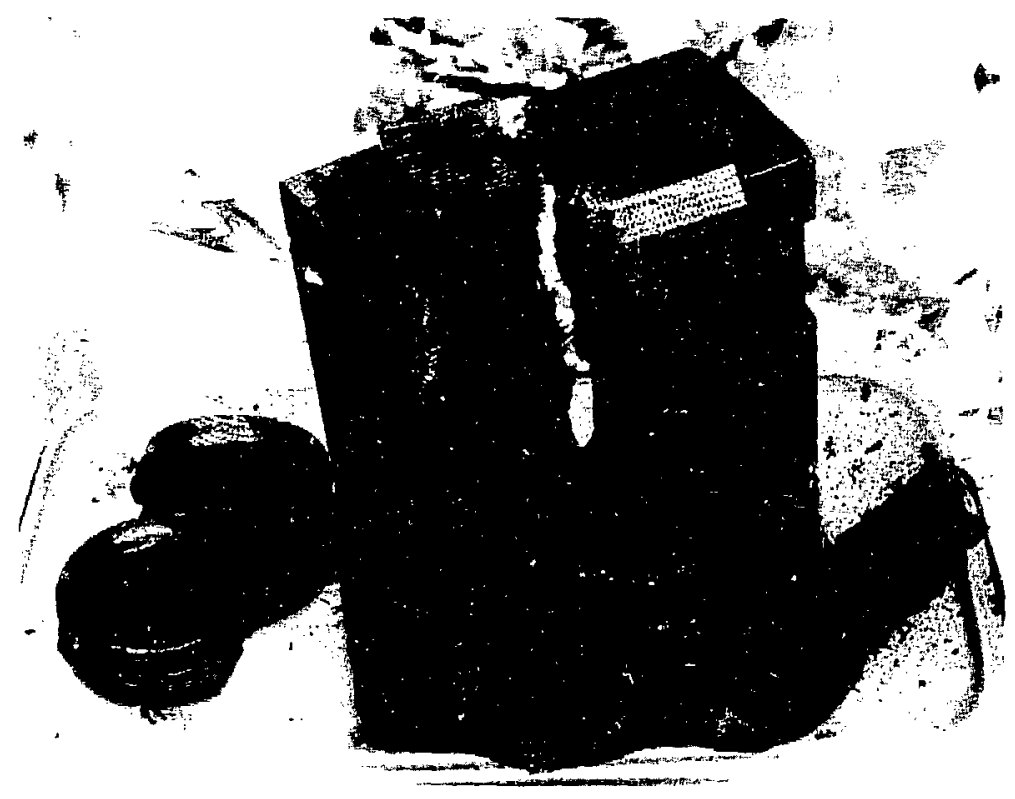

Fig. 31. Damage to the SVT-9 aeroshell was more severe than that observed in the SVT-8 and SVT-7 impacts; $0.4 X$. 


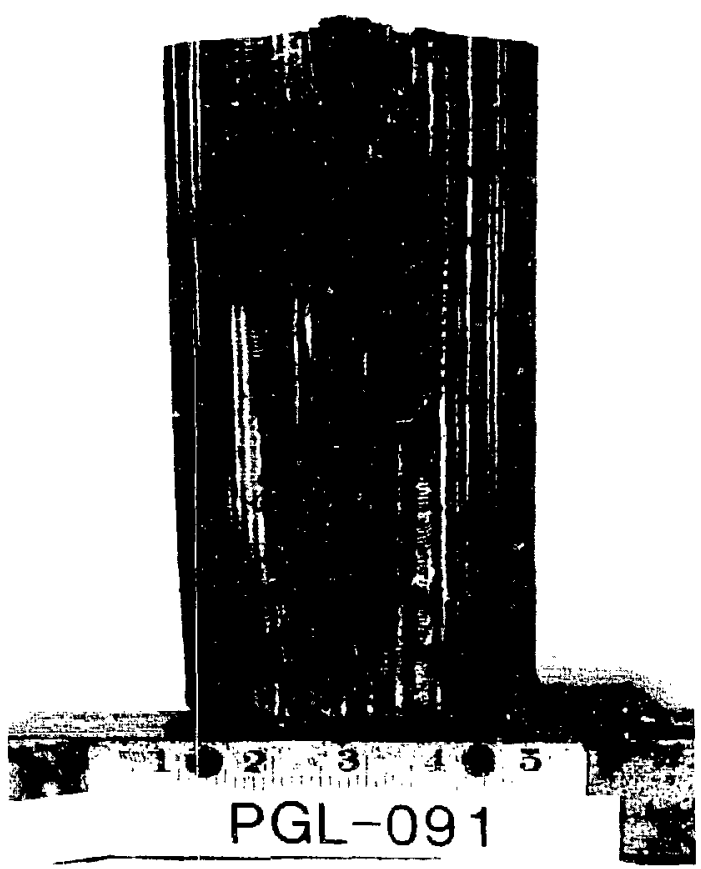

(a)

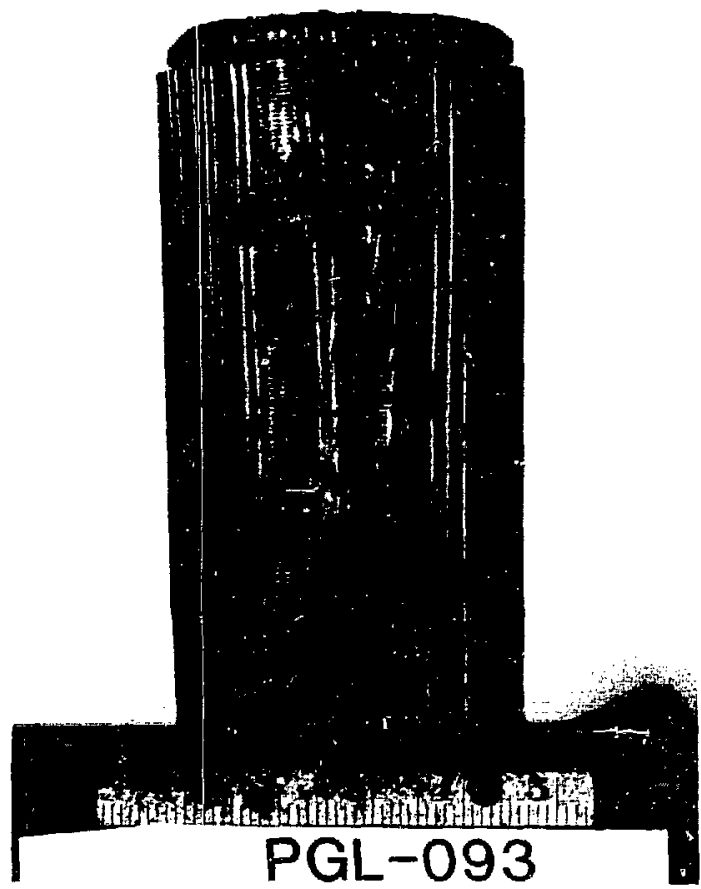

(b)

Fig. 32. Both of the SVT-9 impact shells were cracked on the impact face. (a) Primary GIS and (b) secondary GIS; both at $1.0 \mathrm{X}$. 


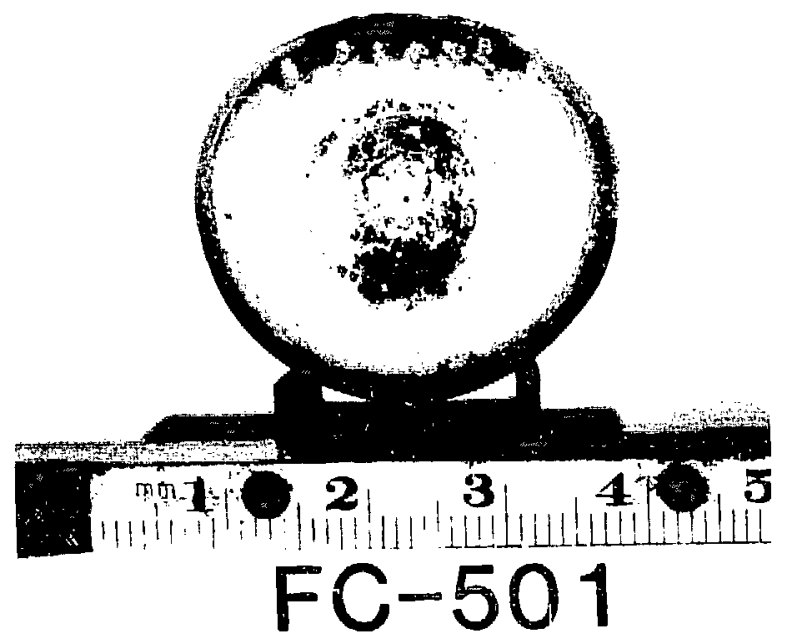

(a)

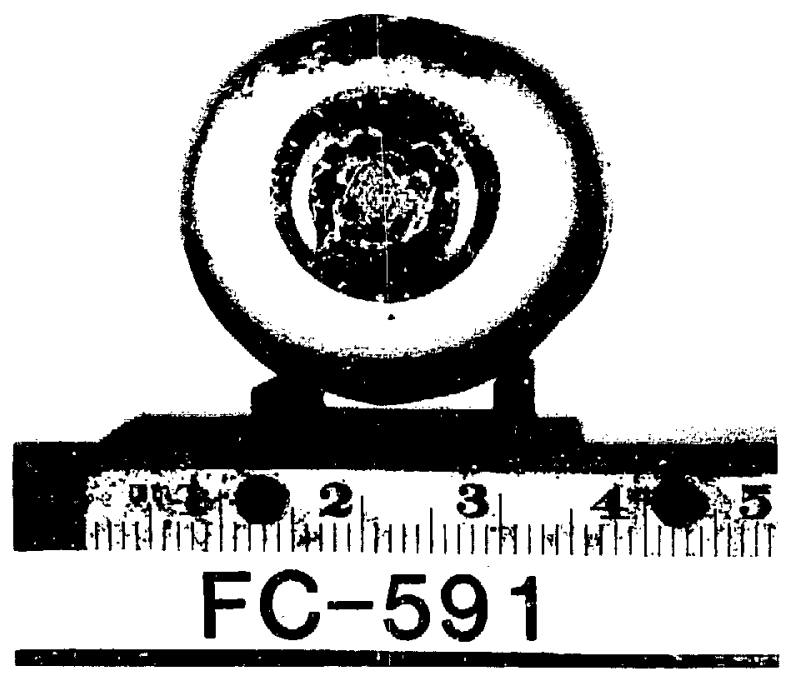

(b)

Fig. 33. Deformation of the SVT-9 fueled clads was symmetric. (a) FC-501 and (b) FC-591; both at 1.5X. 


$$
\|\|
$$




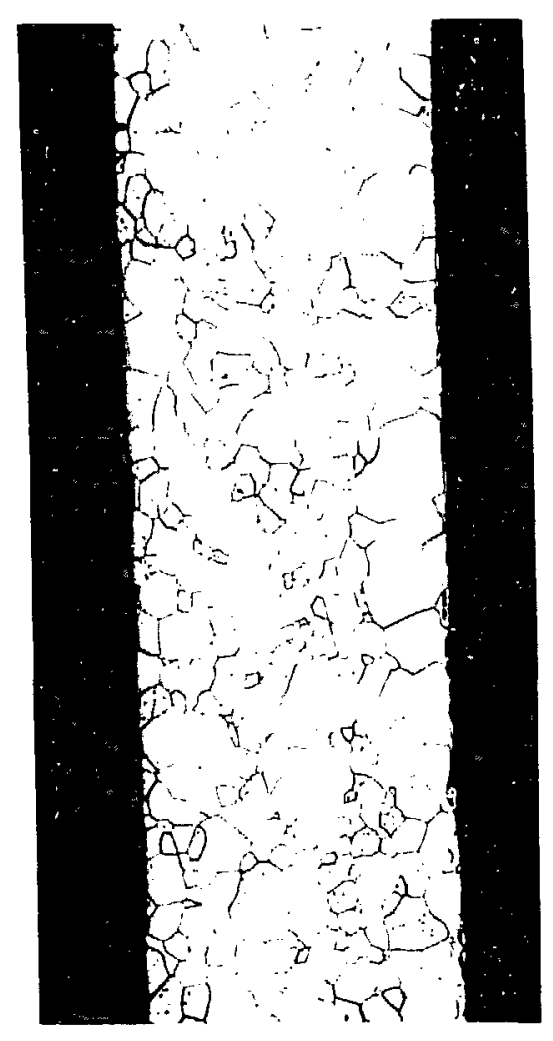

(a)

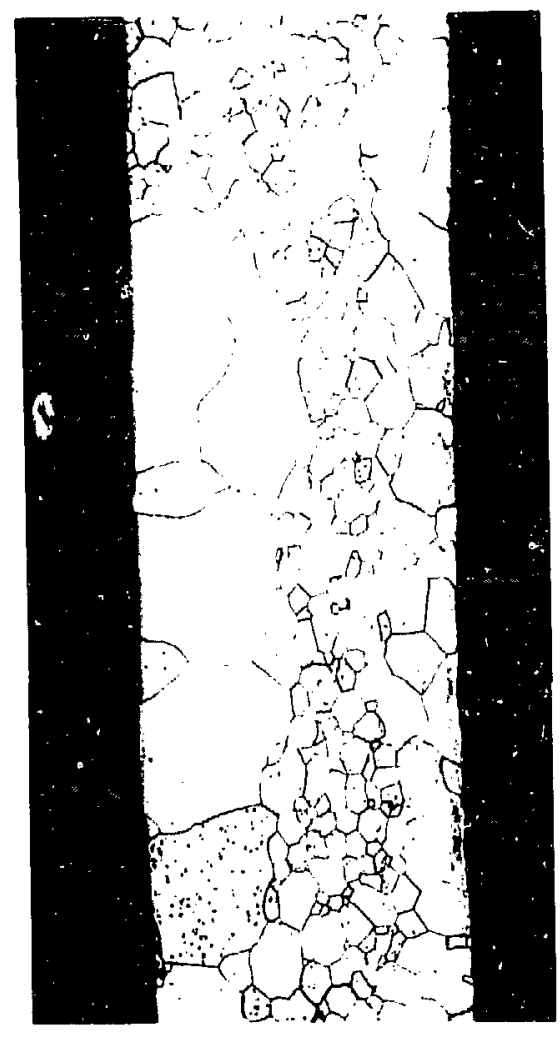

(b)

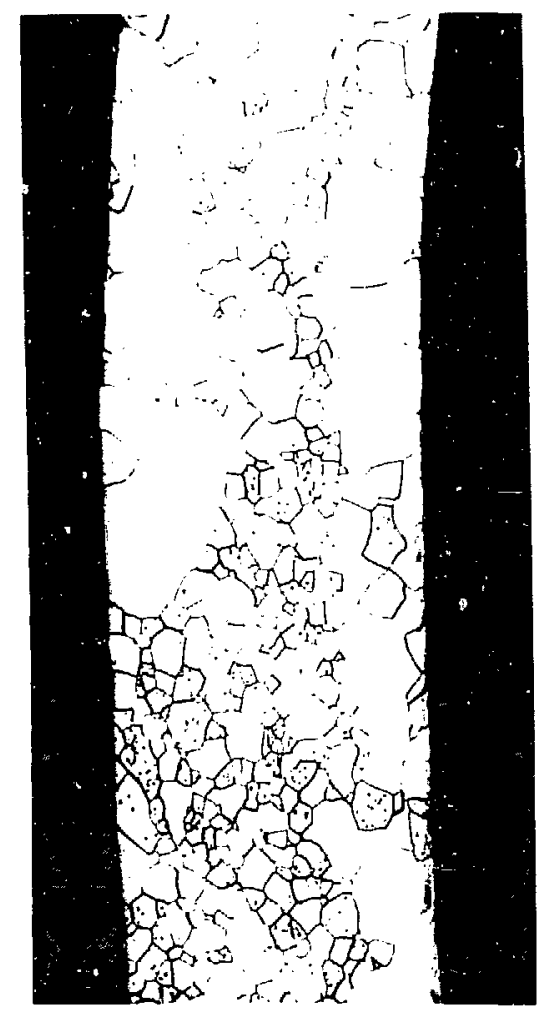

(c)

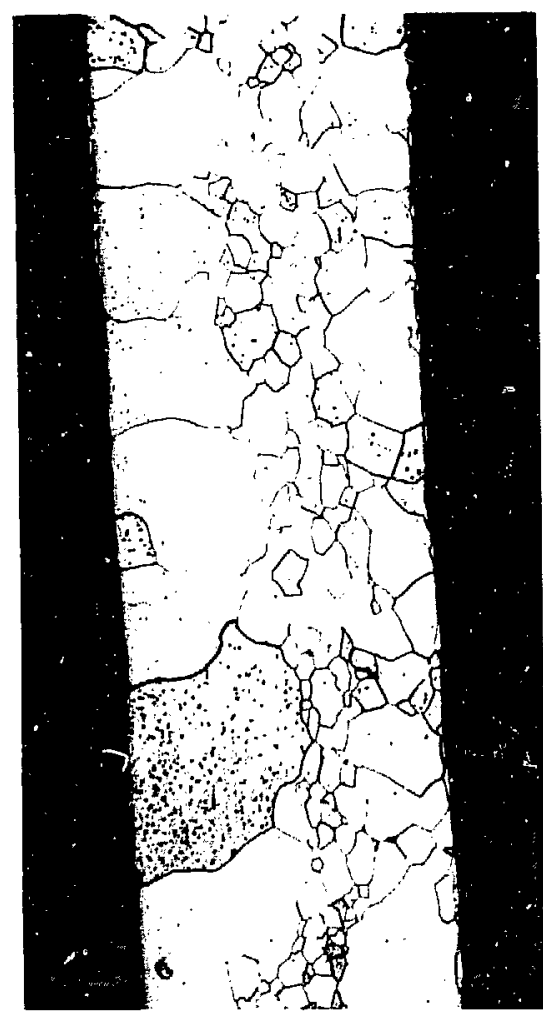

(d)

Fig. 35. The microstructure of the FC-501 vent cup was

variable, with isolated areas of excessive grain growth, (a), (b),

(c), and (d). Etched, with interior surface at left. All at 50X. 


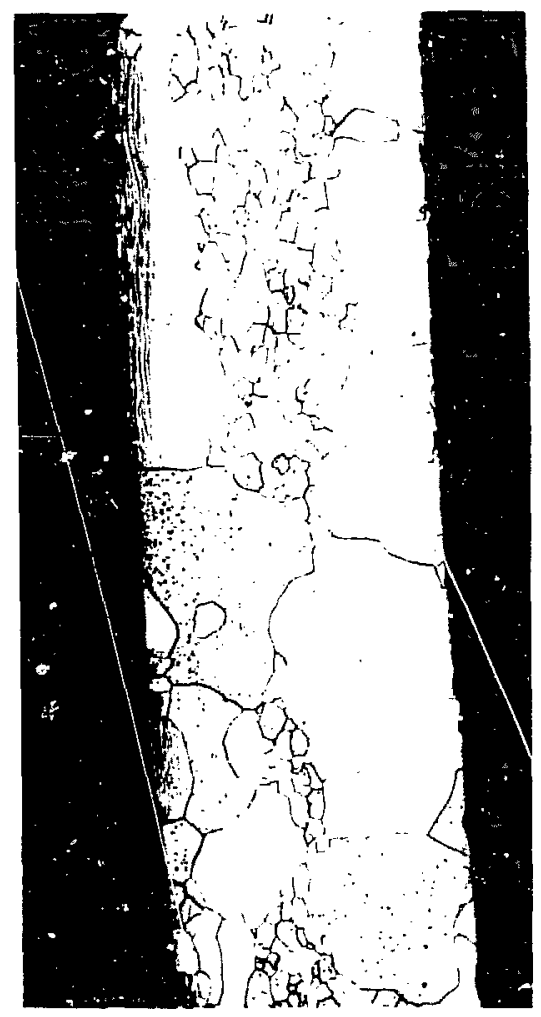

(a)

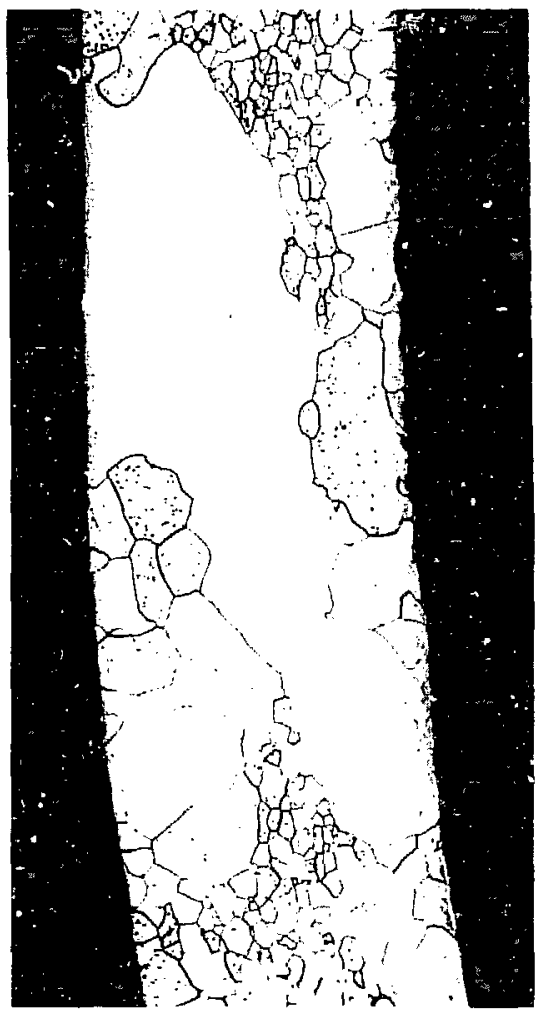

(b)

Fig. 36. The FC-473 and FC-501 shield cups also contained areas of abnormal grain growth. (a) FC-473 shield cup and (b) FC-501 shield cup; etched, with interior surfaces at left. $50 \mathrm{X}$. 

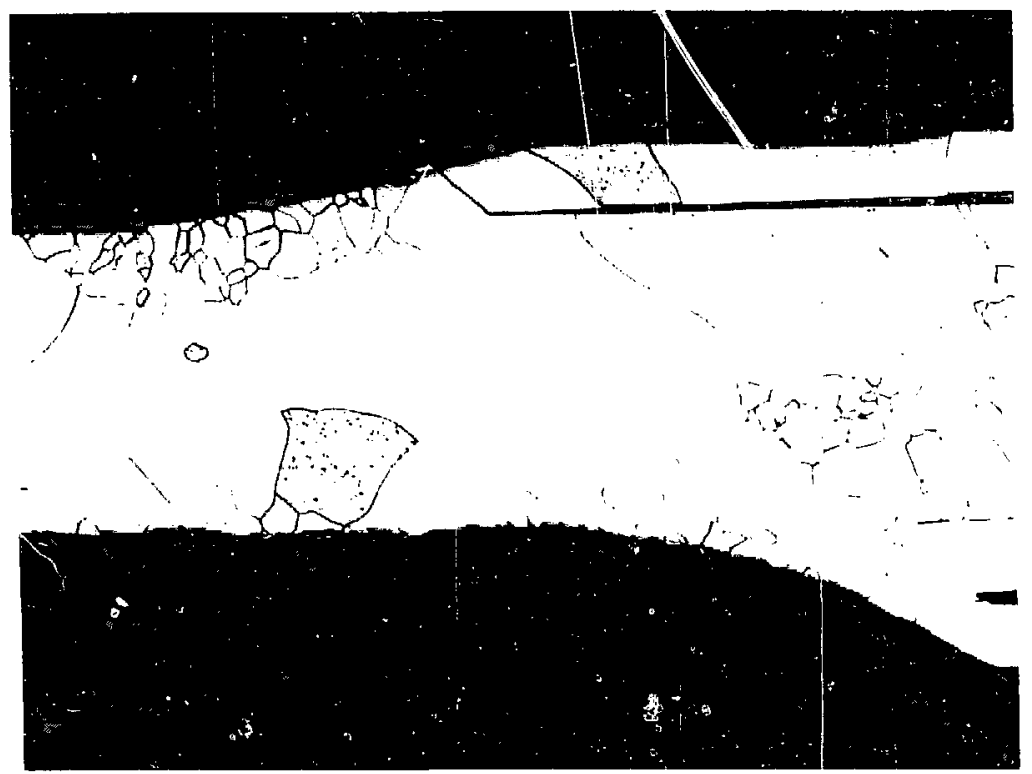

(a)

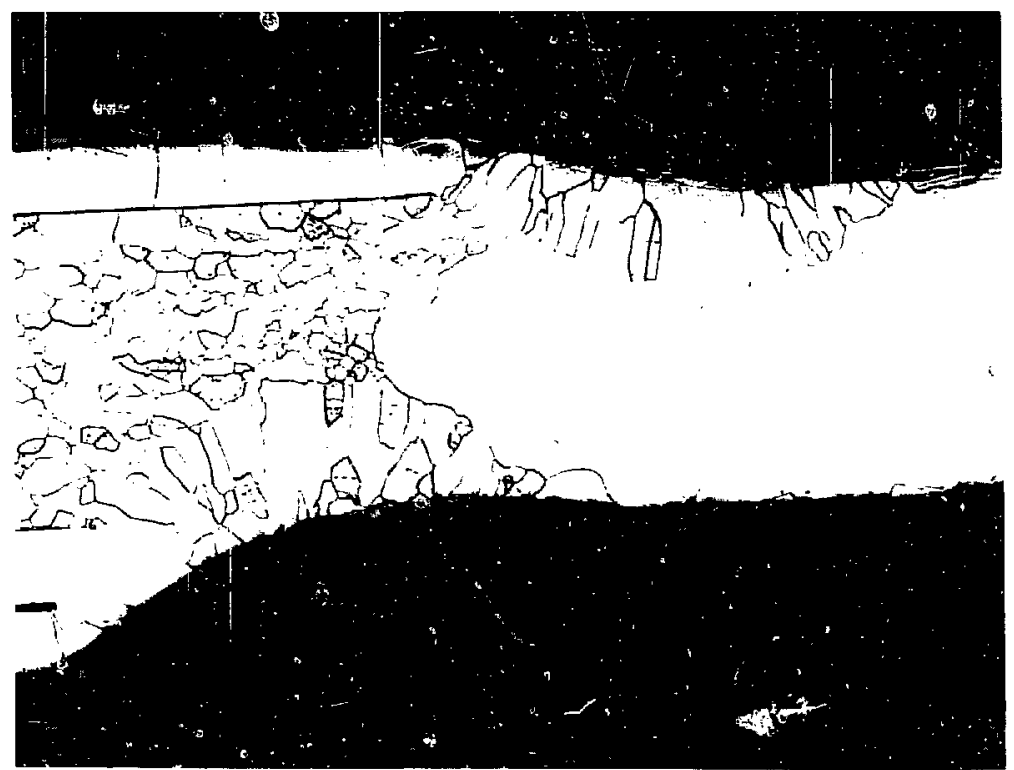

(b)

Fig. 37. Grain coarsening was observed adjacent to the FC-473 vent asse.nbly and decontamination cover welds. (a) Impact face side of vent and (b) opposite side; both at $50 \mathrm{X}$. 


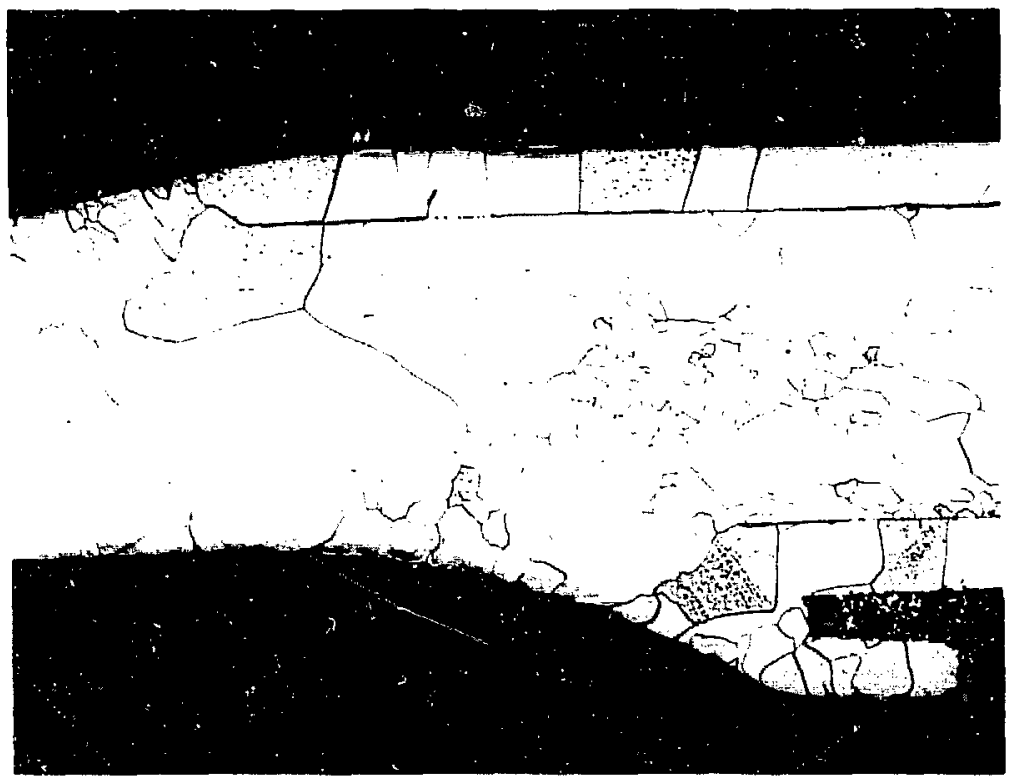

(a)

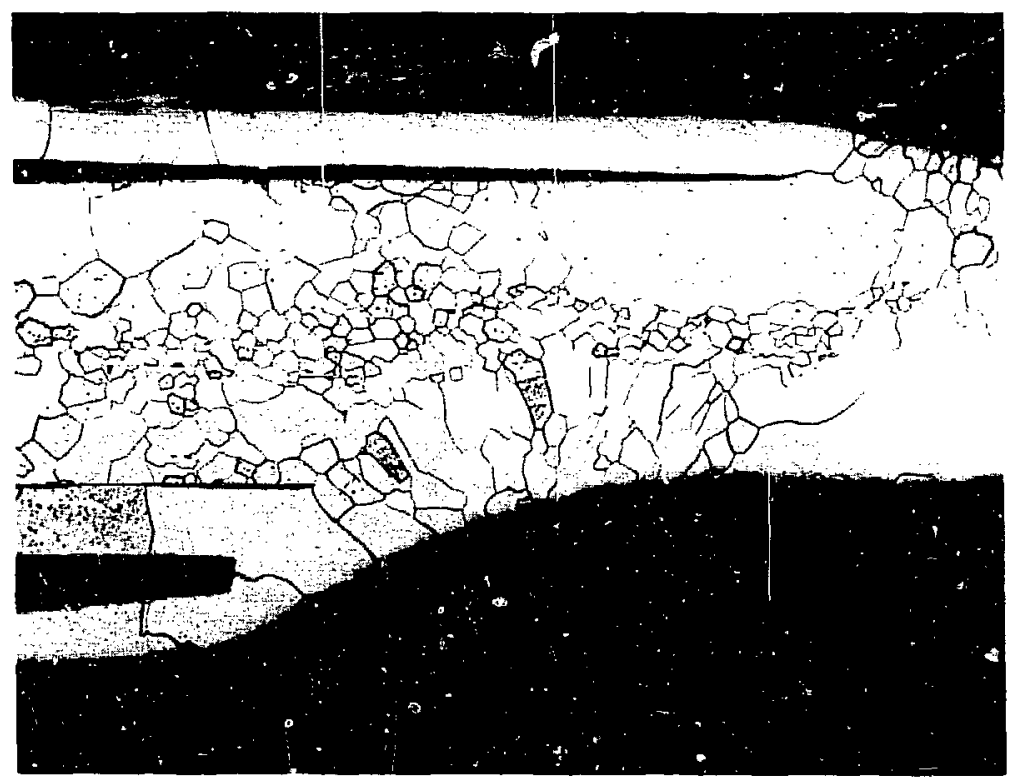

(b)

Fig. 38. Grain growth was also observed near the FC-501 vent assembly and decontamination cover weids. (a) Impact face side of vent and (b) opposite side; both at $50 \mathrm{X}$. 


\section{SVT-10}

The SVT-i0 test module was impacted against a steel. target at $54.3 \mathrm{~m} / \mathrm{s}$ and $975^{\circ} \mathrm{C}$. The module orientation was $\alpha=30^{\circ}, \beta=0^{\circ}, \gamma=0^{\circ}$. Aeroshell breakup was severe (Fig. 39). The aeroshell walls fractured along the axial contact lines of the impact shells, and both GISs were released.

Impact shell damage was moderate. Thin longitudinal cracks, running the length of the impact shell, were observed on the impact face of the leading GIS (Fig. 40 ), and a wide axial crack extended over $75 \%$ of the secondary GIS impact face (Fig. 41). The cap on the secondary GIS had fallen off, and capsule FC-152 was released into the catch tube.

Macroscopic examination of the fueled clads did not reveal any exterior cracks or otner defects. Clad deformation was relatively minor. Although capsule FC-152 was released into the catch tube, it was not significantly more deformed than capsule FC-185, which remained within the secondary GIS. A sharp crease on the FC-152 impact face (Fig. 42) was apparently caused by the differential displacement of a large fuel fragment. Because capsule FC-185 was only minimally deformed and contained no unusual features, it was not examined further.

Examination of the FC-152 closure weld revealed a sight wall misalignment (Fig. 43a) and evidence of incomplete weid penetration (Fig. 43b). The FC-590 closure weld contained significant porosity (Fig. 44a) and had a coarse but otherwise acceptable microstructure (Fig. 44b). The microstructure of the FC-589 closure weld was satisfactory.

Metallographic examination of wall sections removed from the FC-152, FC-589, and FC-590 iridium cups revealed generally fine grained microstructures. The FC-590 weld shield cup, however, contained several coarse-grained sections (Fig. 45). The cup grain sizes ranged from 4 to 16 grains $/ 0.635-\mathrm{mm}$ nominal wall thickness.

Because the fuel capsules experienced only moderate deformation, the capsule vents were unaffected by the impact. No mechanical defects were observed in the FC-589 and FC-590 vent assemblies and neither vent contained intermetaliic or glassy deposits.

Examination of the FC-589 and FC-590 vent cover welds revealed evidence of abnormal grain growth (Figs. 46 and 47). Although large grains were observed near ooth cover welds, capsule FC-590 was worst affected; wall sections adjacent to the FC-590 cover weld contained only 2 grains $/ 0.635-\mathrm{mm}$ nominal wall thickness (Fig. 47b).

AES analyses of specimens removed from the FC-589 and FC-590 iridium cups revealed significant thorium depletion on the inner surfaces. The thorium concentration at the interior of one FC- 589 shield cup section was below the detection limit of the instrument. In addition, high sulfur levels were observed in all of the FC-590 cup specimens.

Spectrographic ar ${ }^{-\cdot 1 y s e s}$ of the SVT-10 iridium cups revealed unusually high iron contents $(275-550 \mathrm{ppm})$ in the FC-589, FC-590, and FC-152 clads. In addition, the FC-590 vent and weld shield cups contained abnormal amounts of copper, aluminum, silicon, chromium, and nickel.

All of the SVT-10 fuel pellets had typical microstructures and fractured in a completely brittle manner. The FC-152 pellet was unusual in that it fractured cleanly along a plane $45^{\circ}$ to the impact face (Fig. 48). Spectrographic and radiochemical analyses of the fuel pellets revealed that the compositions were similar to those of pellets used in previous SVT impacts. However, the calcium contents were somewhat elevated (400-750 ppm) and were above the SRP guideline limits.

\section{v. DISCUSSION}

The test results clearly indicate that the $\alpha=15^{\circ}$, $\beta=0^{\circ}, \gamma=0^{\circ}$ impact orientation prociuced more severe damage than did the $\alpha=30^{\circ}, \beta=0^{\circ}, \gamma=0^{\circ}$ orientation. The differences in capsule deformation illustrate the severity of the $\alpha=15^{\circ}, \beta=0^{\circ}, \gamma=0^{\circ}$ impact; the SVT-7 and SVT-8 postimpact capsule strains (Table V) were approximately $50 \%$ higher than those observed in SVT-9 and SVT-10. In addition, while no failures occurred in SVT-9 and SVT-10, three clads breached in SVT-7. (The breach of capsule FC-39I in

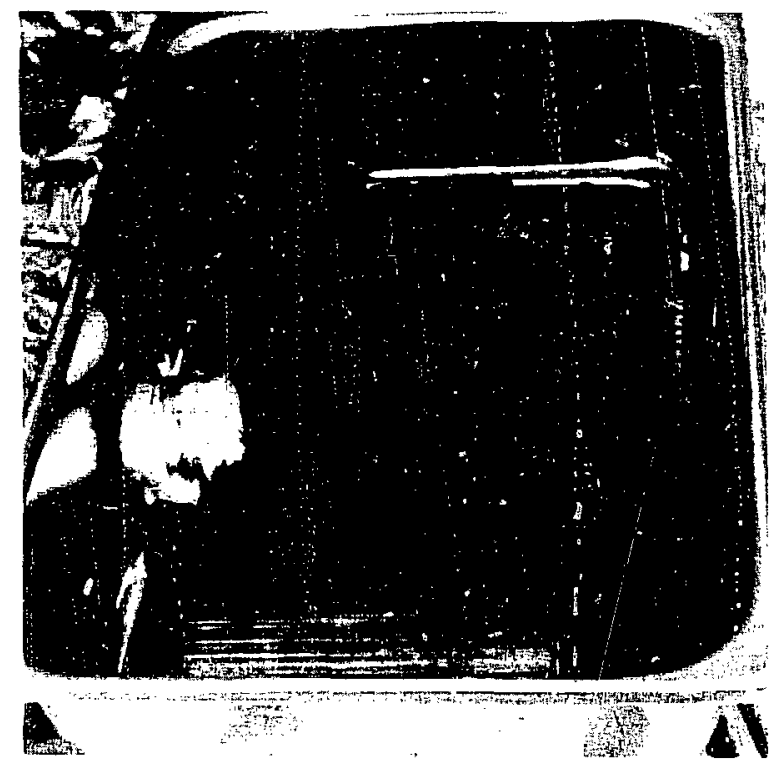

Fig. 39. The SVT-10 aeroshell was severely brokin up; $0.4 \mathrm{X}$. 


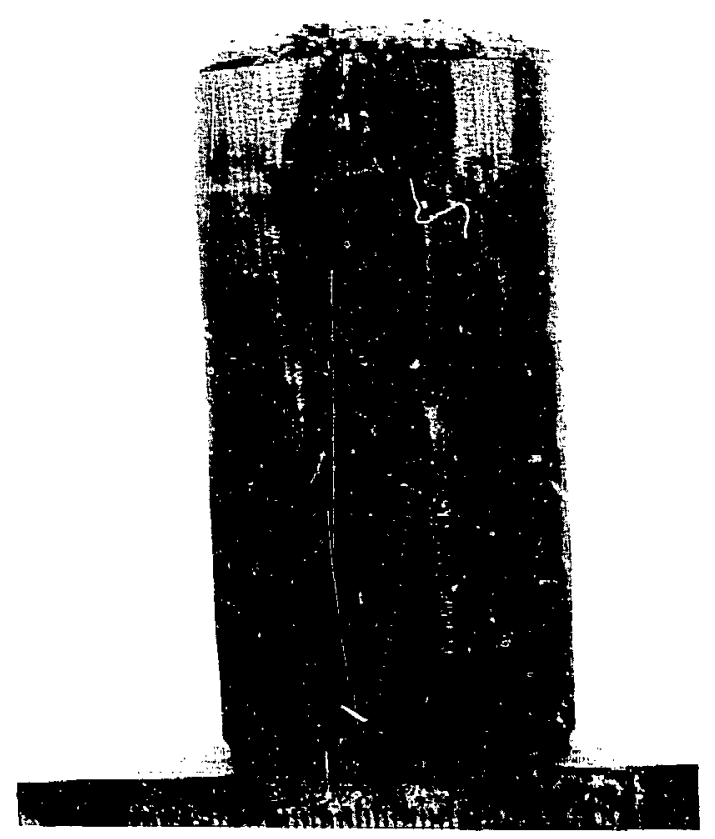

Fig. 40. The impact face of the leading GIS contained numerous longitudinal cracks.

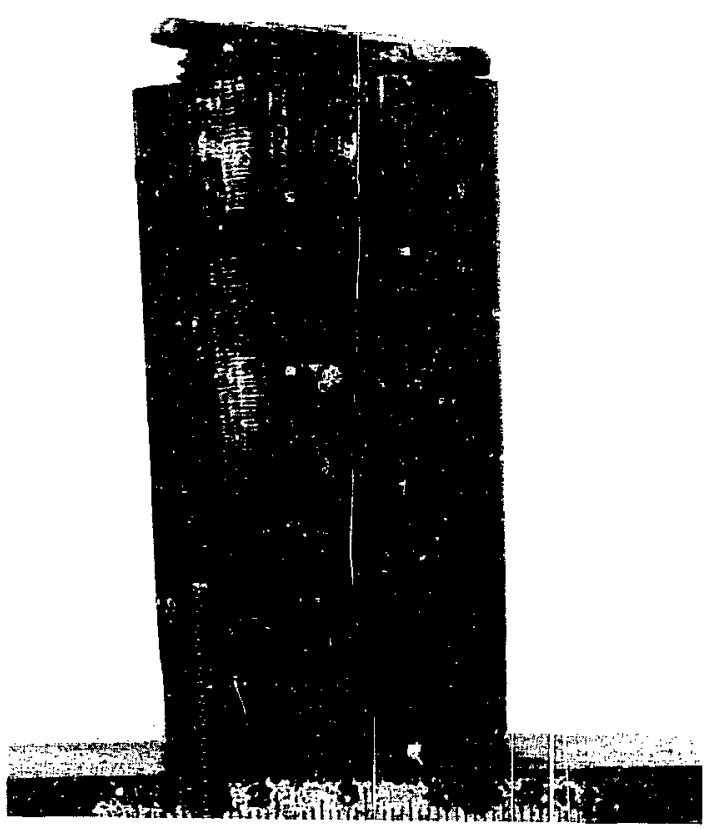

Fig. 41. A wide axial crack was observed on the impact face of the SVT-10 secondary GIS; 1.0X. 


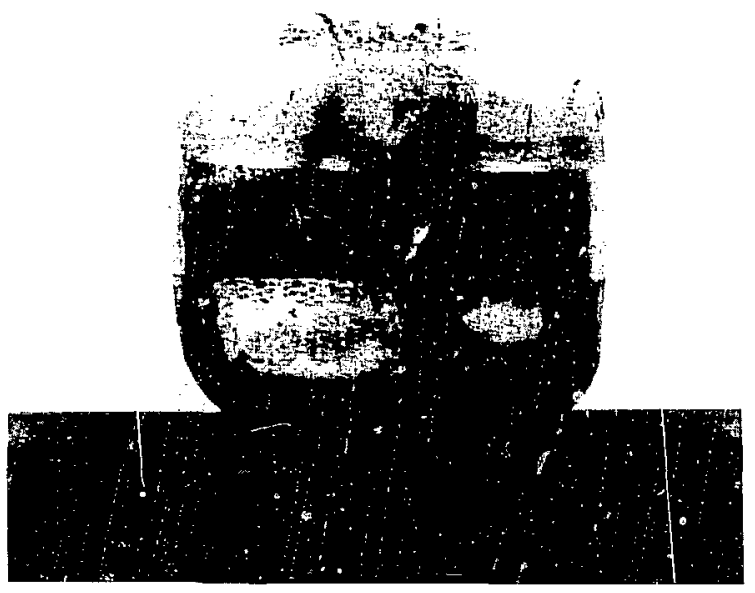

Fig. 42. Although the SVT-10 fueled clads experienced only moderate deformation, a sharp crease was observed on the impact face of capsule FC-152; $1.5 \mathrm{X}$. 


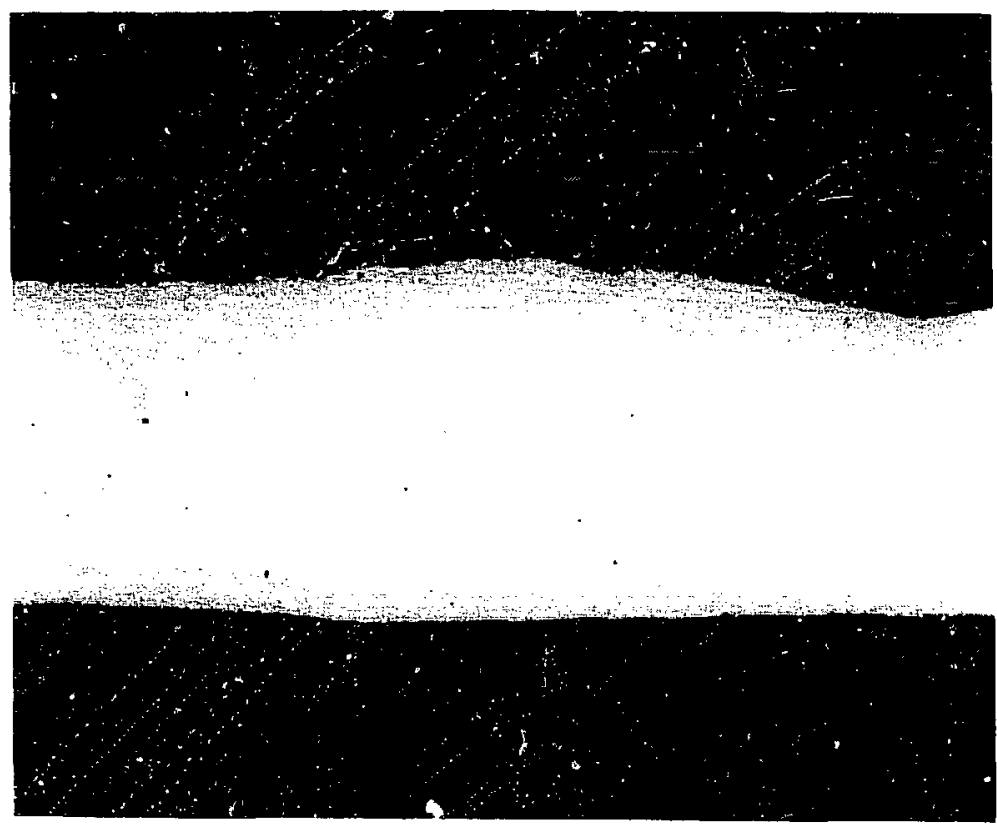

(a)

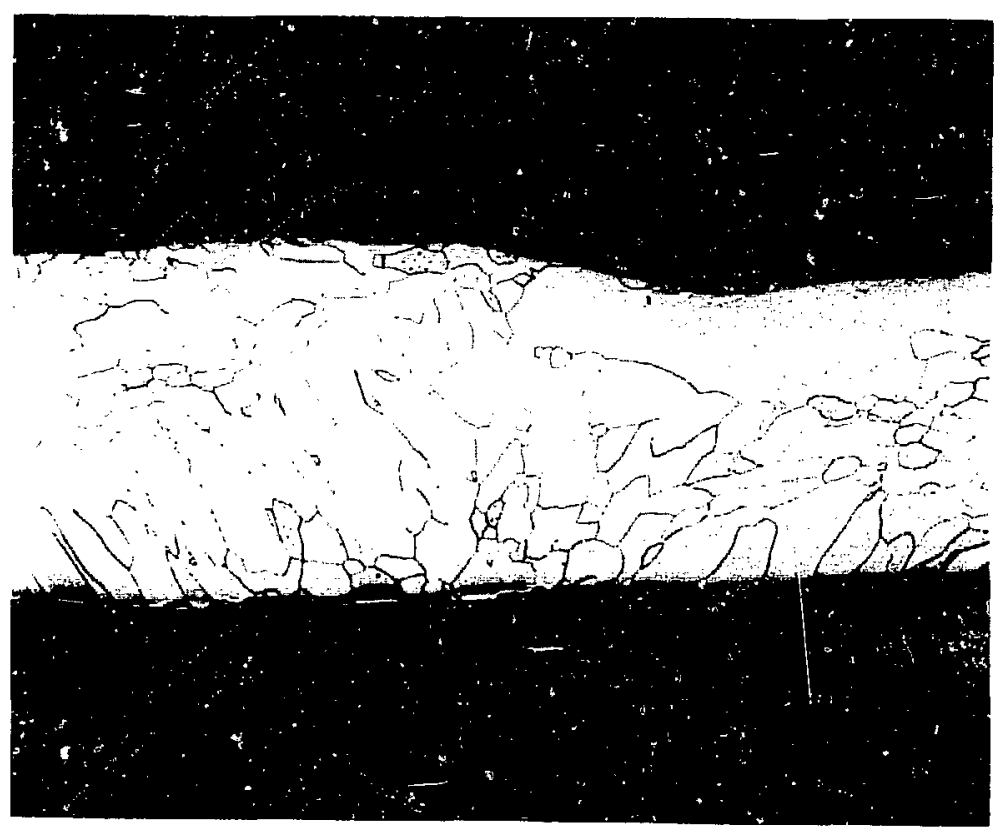

(b)

Fig. 43. The FC-1 52 closure weld was not of good quality. (a) As polished and (b) etched: both at 50X. 


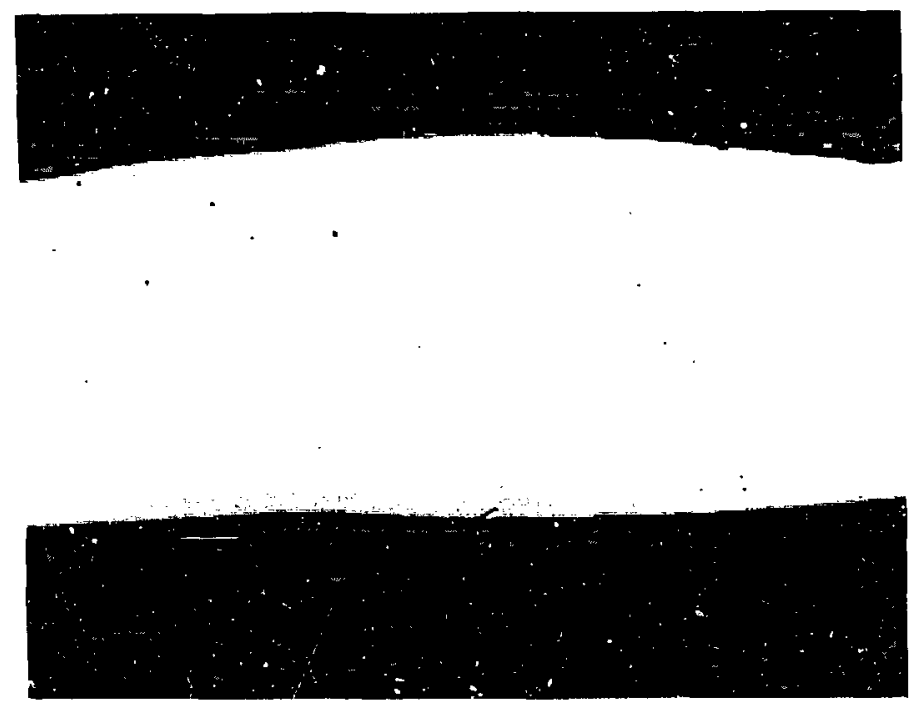

(a)

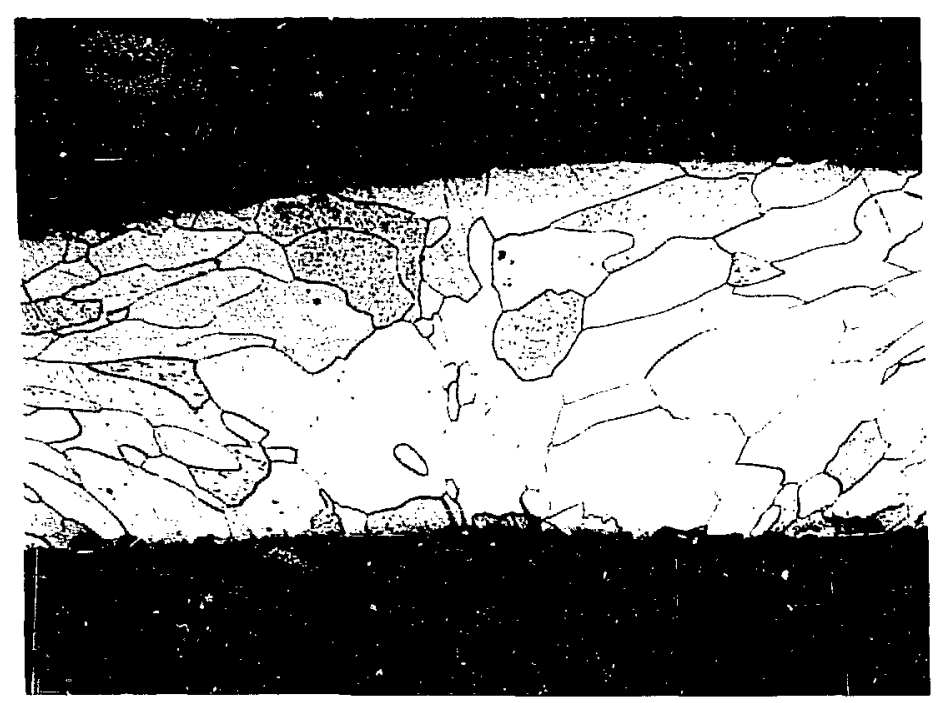

(b)

Fig. 44. The FC- 590 closure weld contained a significant amount of porosity. (ai As polished and (b) etched; both at รถX. 


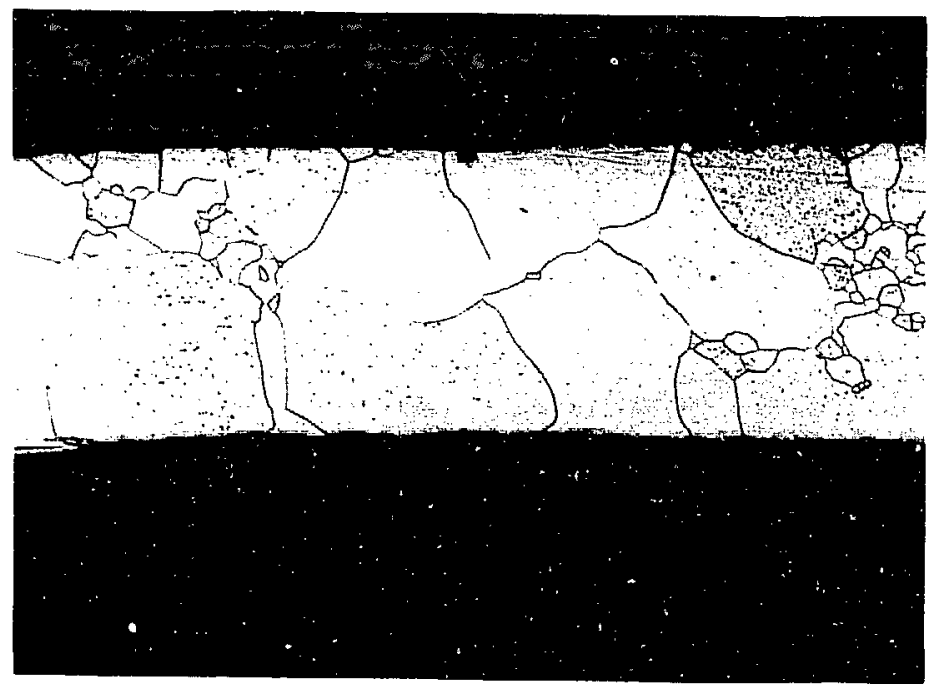

Fig. 45. The FC-590 weld shield cup contained several coarsegrained sections; etched, $50 \mathrm{X}$. 


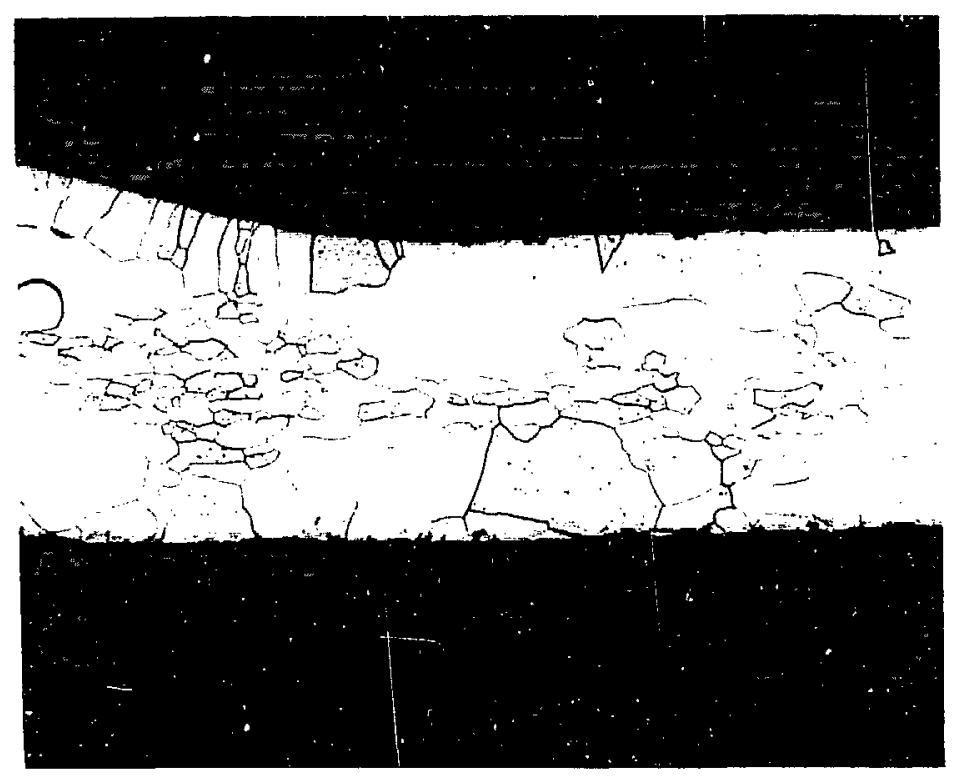

(a)

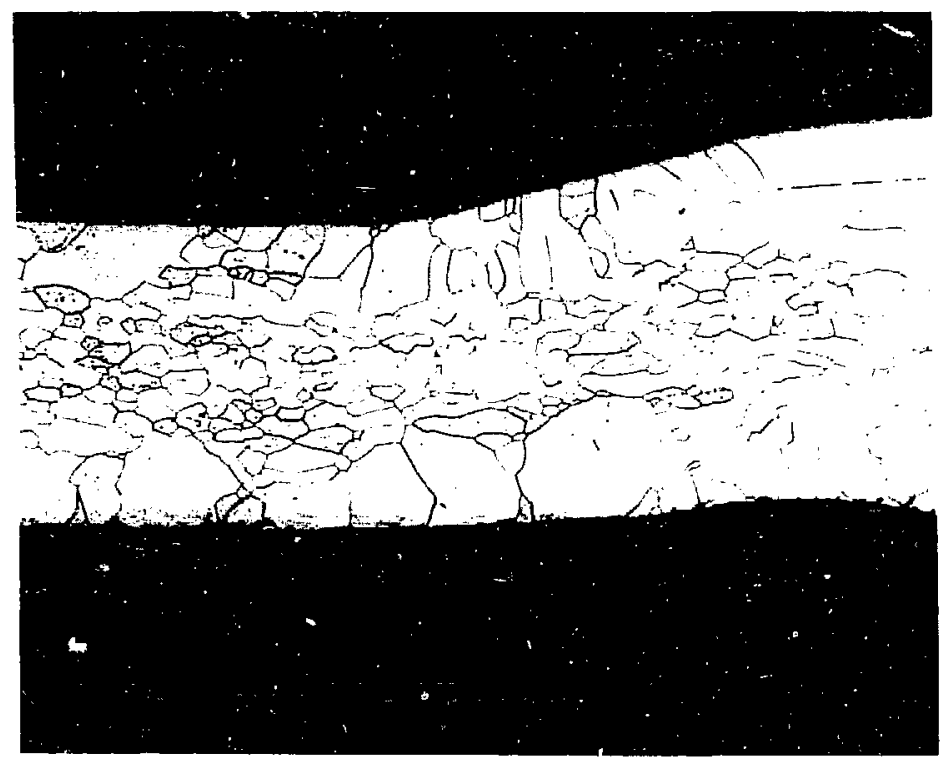

(b)

Fig. 46. Grain coarsening was observed in wall sections adjacent to the FC-589 vent assembly and decontamination cover welds. (a) Impact face side of vent and (b) opposite side; both at $50 \mathrm{X}$. 


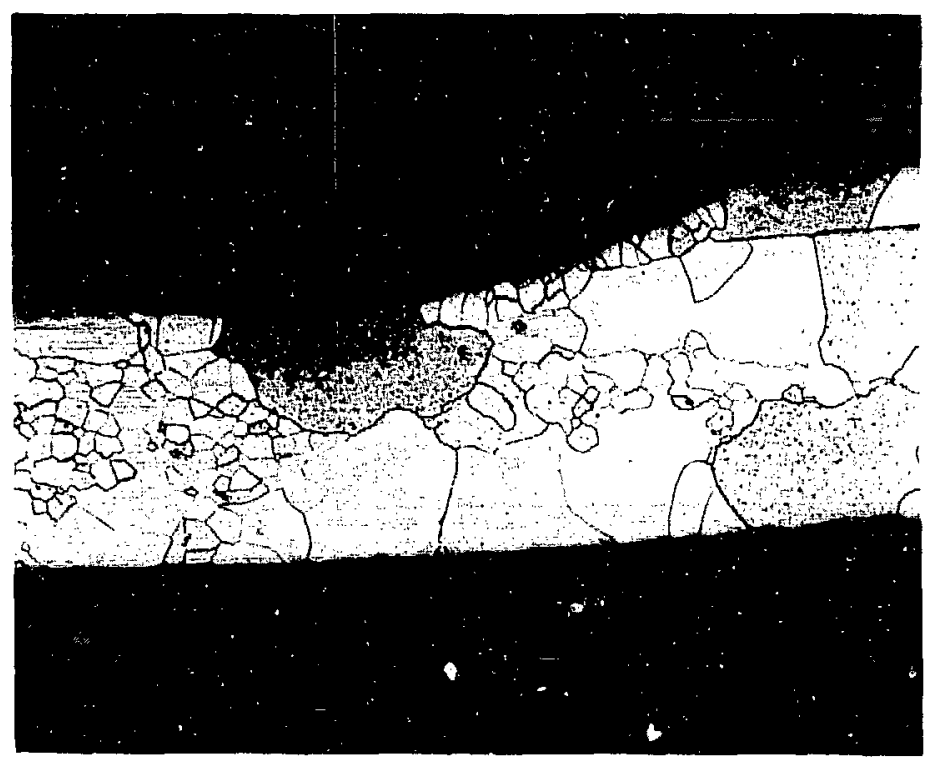

(a)

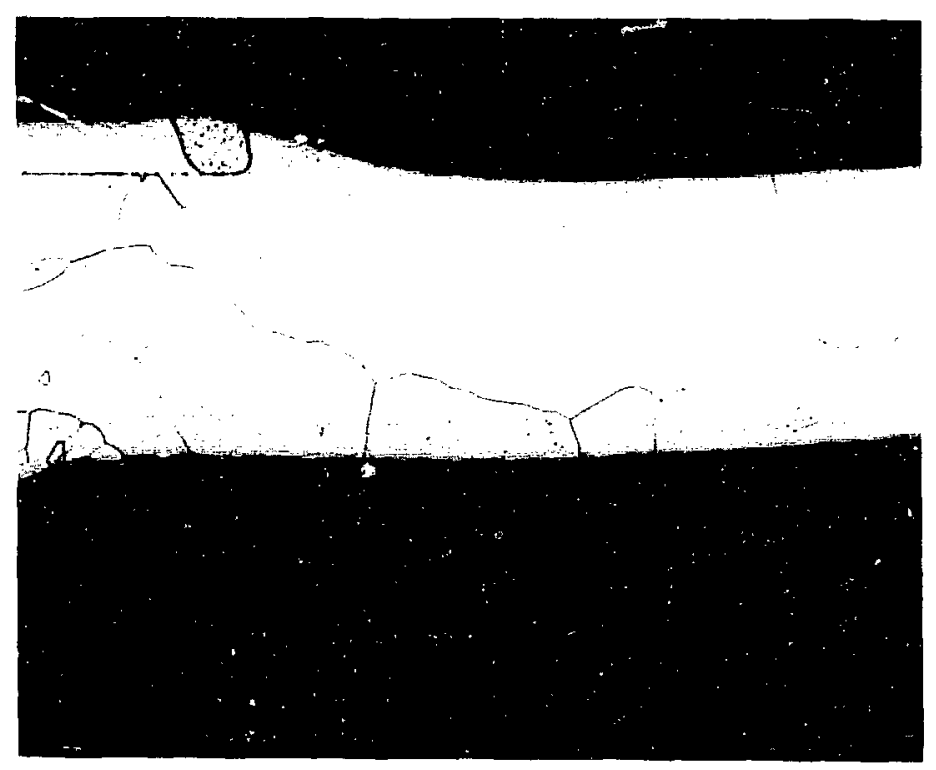

(b)

Fig. 47. Excessive grain growth was also observed in sections adjacent to the FC-590 vent assembly and decontamination cover welds. (a) Impact face side of vent and (b) opposite side; both at $50 \mathrm{X}$. 

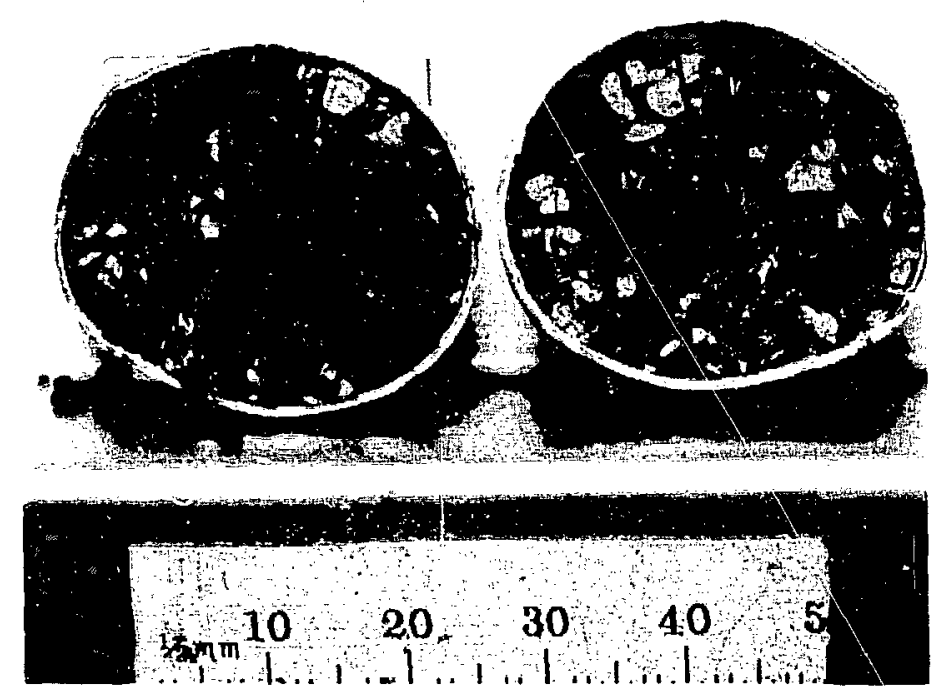

Fig. 48. The FC -152 fuel pellet fractured cieanly along a plane $45^{\circ}$ to the impact facc: $1.5 \mathrm{X}$.

SVT-8 is not considered, because it resulted from the failure of a non-flight-quality weld.) The failure rate of fuel capsules used in the $\alpha=15^{\circ}, \beta=0^{\circ}, \gamma=0^{\circ}$ impacts was $43 \%$.

In SVT-1, one primary (FC-364) and both secondary (FC-112 and FC-165) clads breached. The FC-364 and FC-165 failures (Figs. 5 and 6 ) apparently resulted from the differential displacement and subsequent pushthrough of large fuel fragments. Capsule FC-112 was breached by two small weld cracks (Fig. 7) that probably resulted from the propagation of preexisting weld defects.

Deformation of the SVT-8 clads was significant but somewhat less than that of the SVT-7 clads. Localized iridium deformation was generally mild, and only one secondary clad (FC-391) failed. The FC-391 breach resulted from extensive weld failure (the closure weld fractured over a $120^{\circ}$ arc) and was related to the poor weld quality.

Metallographic examination of FC-391 weld sections revealed significant amounts of porosity (Fig. 24), vent and shield cup misalignment (Fig. 23), and two large voids (Fig. 25) at the edge of the fusion zone. Although the FC-391 weld was not flight qua!tity, the presence of so many defects in a weld with an SRP/NDE rating of only 9.8 is disturbing; fueled clads with SRP/NDE ratings $\leq 8$ are designated as flight quality.

Except for FC -391, the capsule welds were generally acceptable. However, the FC-165 and FC-152 closure welds had unusually coarse grained microstructures.
The FC-165 weld failure occurred in a section containing only 4 grains $/ 0.635-\mathrm{mm}$ wall thickness (Fig. 16); the grains were very elongated and the fracture followed the grain boundaries through the thickness. Although the FC- 152 weld did not fail, the incomplete weld penetration (Fig. 43b) promoted excessive grain growth on the clad interior.

Results of the chemical and microstructural analyses indicate that the iridium cups used in SVT-7 through SVT-10 were similar to those used in previous SVT impacts. The cup microstructures were generally fine grained; with one exception the average grain sizes ranged from 11 to 21 grains $/ 0.635-\mathrm{mm}$ nominal wall thickness. Although all cups contained isolated areas of grain growth, excessively large grains were observed throughout the microstructure of the FC-590 weld shield cup.

The abnormal grain growth observed in the FC-590 weld shield cup and in isolated interior locations of several other cups may have been related to thorium depletion. The AES analyses (Table IX) revealed moderate thorium depletion on the interiors of the SVT-7 and SVT-8 iridium cups. Thorium concentrations on the interiors of three SVT-9 cup samples were very low, and the interiors of all SVT-10 cup samples were severely depleted. In one specimen removed from the FC-589 shield cup, the thorium concentration on the inner surface was below the detection limit of the instrument. Significant amounts of sulfur were also detected in several cup samples. Although the effects of grain 
boundary segregation of sulfur are unclear, sulfur is suspected of promoting grain growth.

Abnormal grain growth was also observed in wall sections adjacent to nearly all the vent assembly and decontamination cover welds. Wall sections adjacent to the vent cover welds on capsules FC 137 and FC-590 container only 2 grains $/ 0.635-\mathrm{mm}$ nominal wall thickness (Figs. 28 and 47). Such excessive grain growth at the same location in several capsules suggests a relation to some aspect of the production process. Whatever the cause, grain coarsening adjacent to the capsule vent could result in a capsule breach in any impact that significantly deformed the vent assembly.

Both the $\alpha=15^{\circ}, \beta=0^{\circ}, \gamma=0^{\circ}$ and $\alpha=30^{\circ}$, $\beta=0^{\circ}, \gamma=0^{\circ}$ module impacts produced negligible vent deformation. Postimpact examination revealed that all capsule vents were relatively undamaged; no mechanical defects were observed in any vent. and all vent frits were free of intermetallic deposits. Although no vent effusates were detected during macroscopic examination, cross sections of the FC-436 and FC-437 capsule vents revealed deposits on the vent exteriors and center holes (Fig. 27). The deposits intergranularly attacked the iridium and promoted excessive grain growth (Figs. 28 and 29). Microprobe analyses revealed that the deposits contained iridium, oxygen, silicon, and tungsten. Although impurities in the fuel would be an obvious silicon source, spectrographic analyses of the FC-436 and FC-437 fuc! pellets indicated low silicon contents.

Spectrographic and radiochemical analyses (Table XI) indicated that the fuel pellets used in SVT-7 through SVT-10 contained acceptable amounts of impurities; however, calcium and aluminum contents of several pellets exceeded the SRP guideline limits. Although the effects of excess aluminum and calcium are unclear, there did not appear to be any relation between chemical composition and impact response. All pellets fractured in a brittle manner. The pellet microstructures were typical of SRP material and similar to the microstructures of the pellets used in previous SVT impacts.

\section{CONCLUSIONS}

(1) The $\alpha=15^{\circ}, \beta=0^{\circ}, \gamma=0^{\circ}$ impact orientation produced markedly more severe clad damage than did the $\alpha=30^{\circ}, \beta=0^{\circ}, \gamma=0^{\circ}$ orientation. The failure rate of fueled clads in the $\alpha=15^{\circ}, \beta=0^{\circ}$, $\gamma=0^{\circ}$ impacts was $43 \%$; no failures occurred in the $\alpha=30^{\circ}, \beta=0^{\circ} . \gamma=0^{\circ}$ impacts.

(2) Fuel fragment push-through was the dominant mode of clad failure. The large breaching cracks observed in SVT-7 were caused by the differential displacement and subsequent push-through of large fuel fragments.
(3) The single clad failure in SVT-8, in which the FC-391 closure weld failed over a $120^{\circ}$ arc, was related to poor weld quality. Because the weld had an SRP/NDE rating of only 9.8, the nondestructive evaluation apparently did not recognize the number or magnitude of weld defects.

(4) Excessively large grains were observed adjacent to nearly all of the vent assembly and decontamination cover welds. Such unusual grain coarsening at the same location in several vents suggests a relation to sume aspect of the production process.

(5) Deposits that promoted grain growth and intergranularly attacked the iridium were seen on the exteriors of the FC-436 and FC-437 capsule vents. The deposits were composed of iridium, oxygen, silicon, and tungsten, and appeared to be a single phase compound.

(6) AES analyses revealed significant thorium depletion on the interiors of nearly all the iridium cups. The cups used in SVT-9 and SVT-10 were worst affected; the thorium content on the interior of one FC-589 shield cup sample was below the detection limit of the instrument.

(7) Chemical and metallographic analyses of the iridium cups and plutonia fuel pellets revealed that they were typical and representative of flight-quality components. The few chemical and microstructural anomalies observed would not have affected the impact responses.

\section{RECOMMENDATIONS}

(1) Because excessive grain growth at the vent cover weld appears to be characteristic s $f$ the clad microstructures, several capsules shr uld be impacted in a configuration that produces significant vent end deformation.

(2) Efforts should be made to identify the mechanism responsible for grain growth at the vent cover welds and to determine the time and temperature required.

\section{ACKNOWLEDGMENTS}

We thank C. Frantz, M. Anstey, and A. Herrera for their test assistance; J. Archuleta and L. Bergamo for their metallography; and D. Garinger for the sieve analyses.

\section{REFERENCES}

1. "Updated Safety Report for the Galileo Mission and the International Solar-Polar Mission," General Electric document GESP-7186, Sec. 3.4 (April 1984)(GPHS Reentry Response). 
2. “GPHS Safety Verification Test Series Procedure Manuial," Los Alamos National Laboratory docuinent MST-5-C-83-19, Sec. 1.0 (April 14, 1983).

D. Pavone, T. G. George, and C. E. Frantz, "GeneralPurpose Heat Source Safety Verification Test Series: SVT.1 Through SVT-6," Los Alamos National Laburatory report LA-10353-MS (June 1985).
4. "Estimates for the Orbital Lifetimes for Galileo and Solar Polar," Applied Physics Laboratory document ATD-RL-82-081/ANSP-L-662 (November 18, 1982).

5. C. E. Frantz, J. W. Taylor, and D. B. Court, "Isotope Fuels Impact Tester (IFIT)," Los Alamos Scientific Laboratory report LA-6013 (January 1976). 